\title{
Selective Release of Aromatic Heterocycles from Ruthenium Tris(2-pyridylmethyl)amine with Visible Light
}

Ao Li, ${ }^{1}$ Jessica K. White, ${ }^{2}$ Karan Arora, ${ }^{1}$ Mackenzie Herroon, ${ }^{3}$ Philip D. Martin, ${ }^{1}$ H. Bernhard Schlegel, ${ }^{1}$ Izabela

Podgorski, ${ }^{3,4}$ Claudia Turro ${ }^{2}$ and Jeremy J. Kodanko ${ }^{1,4^{*}}$

${ }^{1}$ Department of Chemistry, Wayne State University, 5101 Cass Ave, Detroit, MI 48202,

${ }^{2}$ Department of Chemistry and Biochemistry, The Ohio State University, Columbus, Ohio 43210,

${ }^{3}$ Department of Pharmacology, School of Medicine, Wayne State University, Detroit, Michigan 48201,

${ }^{4}$ Barbara Ann Karmanos Cancer Institute, Detroit, Michigan 48201

Corresponding author: jkodanko@chem.wayne.edu

\section{Supporting Information}

(54 Pages)

Part A. General Considerations $\quad 2$

Part B. Experimental Procedures $\quad 2$

Part C. X-ray Crystallographic Analysis 5

Part D. Spectral Data for 1, 2 and $3 \quad 30$

Part E. References $\quad 54$ 


\section{Part A. General Considerations}

All reagents were purchased from commercial suppliers and used as received. NMR spectra were recorded on a Varian FT-NMR Agilent- $400 \mathrm{MHz}$ Spectrometer. IR spectra were recorded on a Nicolet FT-IR spectrophotometer ( $\mathrm{KBr}$ pellet). UV-vis spectra were recorded on a Varian Cary 50 spectrophotometer. All reactions were performed under ambient atmosphere unless otherwise noted. Anaerobic reactions were performed by purging the reaction solutions with Argon.

All the calculations were carried out with B3PW9 $1^{1-3}$ density functional using the Gaussian series of programs. ${ }^{4}$ The SDD basis set and effective core potentia $\left.\right|^{5-7}$ were used for Ru atom, and $6-31 \mathrm{G}(\mathrm{d})$ split-valence polarized basis set ${ }^{8,9}$ for all other atoms. Solvation effect (in acetone) was incorporated by using the SMD implicit solvation model. ${ }^{10}$ Vertical electronic excitations were evaluated using linear response time-dependent density functional theory (TD-DFT). ${ }^{11-13}$

\section{Part B. Experimental Procedures}

Synthesis of 1 [Ru(TPA)(py $\left.)_{2}\right]\left(\mathbf{P F}_{\mathbf{6}}\right)_{2}$. To a solution of TPA ${ }^{14}(20.0 \mathrm{mg}, 0.069 \mathrm{mmol})$ in 6 $\mathrm{mL}$ of dry $\mathrm{MeOH}$ under inert atmosphere in a pressure flask cis- $\left[\mathrm{Ru}\left(\mathrm{Me}_{2} \mathrm{SO}\right)_{4} \mathrm{Cl}_{2}\right](33 \mathrm{mg}$, $0.069 \mathrm{mmol}$ ) was added. The solution was purged with Argon through a submerged needle for $10 \mathrm{~min}$ at room temperature, sealed, and refluxed for $4 \mathrm{~h}$ under inert atmosphere. The color of the reaction mixture changed from pale yellow to dark yellow. The reaction mixture was cooled to room temperature and concentrated under reduced pressure. A 1:1 mixture of pyridine and $\mathrm{H}_{2} \mathrm{O}(6 \mathrm{~mL})$ was added and the reaction mixture was refluxed for $16 \mathrm{~h}$ under inert atmosphere. The reaction mixture was cooled to room temperature. Ice cold water (15 $\mathrm{mL})$ and $\mathrm{NH}_{4} \mathrm{PF}_{6}(15 \mathrm{mg})$ was added to the reaction mixture, resulting in the formation of yellow precipitate which was filtered, washed with ice cold water $(200 \mathrm{~mL})$ and dried under reduced pressure to give the title complex as a yellow solid $(47 \mathrm{mg}, 80 \%)$ : ${ }^{1} \mathrm{H}$ NMR $\left(400 \mathrm{MHz}\left(\mathrm{CD}_{3}\right)_{2} \mathrm{CO}\right) \delta 8.85-8.82(\mathrm{~m}, 4 \mathrm{H}), 8.75(\mathrm{~d}, 1 \mathrm{H}, J=6.0 \mathrm{~Hz}), 8.19-8.14(\mathrm{~m}, 3 \mathrm{H}), 7.99-$ $7.95(\mathrm{~m}, 3 \mathrm{H}), 7.75-7.69(\mathrm{~m}, 5 \mathrm{H}), 7.53-7.49(\mathrm{t}, 2 \mathrm{H}, J=6.4 \mathrm{~Hz}), 7.42-7.38(\mathrm{~m}, 3 \mathrm{H}), 7.27(\mathrm{t}$, $1 \mathrm{H}, J=6.4 \mathrm{~Hz}), 5.26(\mathrm{~d}, 2 \mathrm{H}, J=16.0 \mathrm{~Hz}), 5.05(\mathrm{~d}, 2 \mathrm{H}, J=16.0 \mathrm{~Hz}), 4.90(\mathrm{~s}, 2 \mathrm{H}) ; \mathrm{IR}(\mathrm{KBr})$ $v_{\max }\left(\mathrm{cm}^{-1}\right) 3750,3649,3421,3121,2920,1607,1569,1541,1485,1447,1391,1314,1286$, 1217, 1160, 1065, 993, 972, 958, 882, 836, 772, 758, 740, 712, 698; ESMS calcd for $\mathrm{C}_{28} \mathrm{H}_{28} \mathrm{~F}_{6} \mathrm{~N}_{6} \mathrm{PRu}\left(\mathrm{M}^{+1}\right)$ 695.11, found 695.23; UV-vis $\lambda_{\max }=355 \mathrm{~nm}\left(\varepsilon=10,800 \mathrm{M}^{-1} \mathrm{~cm}^{-1}\right)$; Anal. Calcd for $\mathrm{C}_{28} \mathrm{H}_{28} \mathrm{~F}_{12} \mathrm{~N}_{6} \mathrm{P}_{2} \mathrm{Ru}$ : $\left(1 \cdot 1 / 3 \mathrm{Et}_{2} \mathrm{O}\right) \mathrm{C}, 40.76 ; \mathrm{H}, 3.65 ; \mathrm{N}, 9.72$. Found: $\mathrm{C}, 40.31 ; \mathrm{H}$, $3.43 ; \mathrm{N}, 9.74$.

Synthesis of $2\left[\mathbf{R u}(\mathbf{T P A})(\mathbf{N A})_{2}\right]\left(\mathbf{P F}_{\mathbf{6}}\right)_{2}$. To a solution of TPA $(20.0 \mathrm{mg}, 0.069 \mathrm{mmol})$ in 10 $\mathrm{mL}$ of dry $\mathrm{MeOH}$ under inert atmosphere in a pressure flask cis- $\left[\mathrm{Ru}\left(\mathrm{Me}_{2} \mathrm{SO}\right)_{4} \mathrm{Cl}_{2}\right](33 \mathrm{mg}$, $0.069 \mathrm{mmol}$ ) was added. The solution was purged with Argon through a submerged needle for $10 \mathrm{~min}$ at room temperature, sealed, and refluxed for $4 \mathrm{~h}$ under inert atmosphere. The color of the reaction mixture changed from pale yellow to dark yellow. The reaction mixture was cooled to room temperature and concentrated under reduced pressure. A 3:5 mixture of acetone: $\mathrm{H}_{2} \mathrm{O}(10 \mathrm{~mL})$ and nicotinamide $(84 \mathrm{mg}, 0.69 \mathrm{mmol})$ was added and the reaction 
mixture was refluxed for $16 \mathrm{~h}$ under inert atmosphere. The reaction mixture cooled to room temperature. Ice cold water $(15 \mathrm{~mL})$ and $\mathrm{NH}_{4} \mathrm{PF}_{6}(15 \mathrm{mg})$ was then added to the reaction mixture, resulting in the formation of yellow precipitate which was filtered, washed with ice cold water $(200 \mathrm{~mL})$ and dried under reduced pressure. The precipitate was purified by silica gel chromatography (acetone) and the title complex was obtained as a yellow solid (48 $\mathrm{mg}$, $75 \%):{ }^{1} \mathrm{H}$ NMR $\left(400 \mathrm{MHz}\left(\mathrm{CD}_{3}\right)_{2} \mathrm{CO}\right) \delta 9.36(\mathrm{~s}, 1 \mathrm{H}), 8.94(\mathrm{~d}, 1 \mathrm{H}, J=5.2 \mathrm{~Hz}), 8.84(\mathrm{t}, 2 \mathrm{H}, J$ $=6.4 \mathrm{~Hz}), 8.78(\mathrm{~d}, 1 \mathrm{H}, J=5.6 \mathrm{~Hz}), 8.61(\mathrm{~d}, 1 \mathrm{H}, J=8.0 \mathrm{~Hz}), 8.56(\mathrm{~s}, 1 \mathrm{H}), 8.36(\mathrm{~d}, 1 \mathrm{H}, J=$ $8.0 \mathrm{~Hz}), 8.25(\mathrm{~d}, 1 \mathrm{H}, J=6.0 \mathrm{~Hz}), 7.99-7.96(\mathrm{~m}, 3 \mathrm{H}), 7.83-7.79(\mathrm{~m}, 1 \mathrm{H}), 7.75-7.71(\mathrm{~m}, 4 \mathrm{H})$, 7.55-7.44 (m, 3H), 7.41-7.39 (d, 1H, J=7.6 Hz), $7.28(\mathrm{t}, 1 \mathrm{H}, J=7.2 \mathrm{~Hz}), 7.15(\mathrm{~s}, 1 \mathrm{H}), 6.94$ $(\mathrm{s}, 1 \mathrm{H}), 5.27(\mathrm{dd}, 2 \mathrm{H}, J=16.0 \mathrm{~Hz}), 5.15-5.05(\mathrm{dd}, 2 \mathrm{H}, J=16.0 \mathrm{~Hz}), 4.94(\mathrm{~s}, 2 \mathrm{H})$; IR (KBr) $v_{\max }\left(\mathrm{cm}^{-1}\right) 3649,3382,3305,2919,2360,1813,1687,1608,1573,1478,1398,1313,1223$, 1199, 1161, 836, 763, 739, 701, 624; ESMS calcd for $\mathrm{C}_{30} \mathrm{H}_{30} \mathrm{~F}_{6} \mathrm{~N}_{8} \mathrm{O}_{2} \mathrm{PRu}\left(\mathrm{M}^{+1}\right)$ 781.12, found 780.95; UV-vis $\lambda_{\max }=385 \mathrm{~nm}\left(\varepsilon=11,200 \mathrm{M}^{-1} \mathrm{~cm}^{-1}\right)$. Anal. Calcd for $\mathrm{C}_{30} \mathrm{H}_{30} \mathrm{~F}_{12} \mathrm{~N}_{8} \mathrm{O}_{2} \mathrm{P}_{2} \mathrm{Ru}$ : $\left(2 \cdot 1 / 7 \mathrm{Et}_{2} \mathrm{O}\right) \mathrm{C}, 39.22 ; \mathrm{H}, 3.38 ; \mathrm{N}, 11.97$. Found: C, 38.46; H, 3.38; N, 11.63.

Synthesis of 3 [Ru(TPA)(Im) $\left.)_{2}\right]\left(\mathbf{P F}_{\mathbf{6}}\right)_{2}$. To a solution of TPA $(20.0 \mathrm{mg}, 0.069 \mathrm{mmol})$ in $6 \mathrm{~mL}$ of dry $\mathrm{MeOH}$ under inert atmosphere in a pressure flask cis-[Ru( $\left.\left.\mathrm{Me}_{2} \mathrm{SO}\right)_{4} \mathrm{Cl}_{2}\right](33.3 \mathrm{mg}$, $0.069 \mathrm{mmol}$ ) was added. The solution was purged with Argon through a submerged needle for $10 \mathrm{~min}$ at room temperature, sealed, and refluxed for $4 \mathrm{~h}$ under inert atmosphere. The color of the reaction mixture changed from pale yellow to dark yellow. The reaction mixture was cooled to room temperature and concentrated under reduced pressure. Imidazole (47 mg, $0.69 \mathrm{mmol})$ and $1: 1$ mixture of $\mathrm{EtOH}$ and $\mathrm{H}_{2} \mathrm{O}(6 \mathrm{~mL})$ was added and the reaction mixture was refluxed for $16 \mathrm{~h}$ under inert atmosphere. The reaction mixture was cooled to room temperature. Ice cold water $(15 \mathrm{~mL})$ and $\mathrm{NH}_{4} \mathrm{PF}_{6}(15 \mathrm{mg})$ was then added to the reaction mixture, resulting in the formation of yellow precipitate which was filtered, washed with ice cold water $(200 \mathrm{~mL})$ and dried under reduced pressure to give title complex as a yellow solid (51 mg, $90 \%):{ }^{1} \mathrm{H}$ NMR $\left(400 \mathrm{MHz}\left(\mathrm{CD}_{3}\right)_{2} \mathrm{CO}\right) \delta 8.95(\mathrm{~d}, 1 \mathrm{H}, J=5.6 \mathrm{~Hz}), 8.77(\mathrm{~d}, 2 \mathrm{H}, J=$ $5.2 \mathrm{~Hz}), 7.90-7.85(\mathrm{~m}, 3 \mathrm{H}), 7.65-7.61(\mathrm{~m}, 3 \mathrm{H}), 7.56(\mathrm{~s}, 1 \mathrm{H}), 7.43-7.40(\mathrm{t}, 2 \mathrm{H}, J=6.4 \mathrm{~Hz})$, 7.32-7.22 (m, 6H), $6.41(\mathrm{~s}, 1 \mathrm{H}), 5.08(\mathrm{~d}, 2 \mathrm{H}, J=15.2 \mathrm{~Hz}), 4.85-4.80(\mathrm{~m}, 4 \mathrm{H}) ; \mathrm{IR}(\mathrm{KBr}) v_{\max }$ $\left(\mathrm{cm}^{-1}\right)$ 3649, 3432, 3151, 2975, 2933, 2382, 2349, 1772, 1698, 1647, 1605, 1541, 1446, 1387, 1327, 1307, 1285, 1259, 1159, 1103, 1072, 1026, 990, 970, 838, 761, 738, 693, 664, 610; ESMS calcd for $\mathrm{C}_{24} \mathrm{H}_{26} \mathrm{~F}_{6} \mathrm{~N}_{8} \mathrm{PRu}\left(\mathrm{M}^{+1}\right) 673.10$, found 672.78 ; UV-vis $\lambda_{\max }=425 \mathrm{~nm}(\varepsilon=$ $\left.10,300 \mathrm{M}^{-1} \mathrm{~cm}^{-1}\right)$; Anal. Calcd for $\mathrm{C}_{24} \mathrm{H}_{26} \mathrm{~F}_{12} \mathrm{~N}_{8} \mathrm{P}_{2} \mathrm{Ru}$ : (3·1/3 Et $\left.\mathrm{O}\right) \mathrm{C}, 36.13 ; \mathrm{H}, 3.51 ; \mathrm{N}, 13.30$. Found: C, 35.92; H, 3.55; N, 13.03 .

Photolysis and Quantum Yields. Photolysis and ligand exchange quantum yield experiments were performed using a $150 \mathrm{~W}$ Xe arc lamp (USHIO) in a MilliArc lamp housing unit. The lamp was powered by an LPS-220 power supply equipped with an LPS221 igniter (all from PTI). Bandpass filters (Thorlabs) and long pass filters (CVI Melles Griot) were used to select the appropriate excitation wavelengths. A $395 \mathrm{~nm}$ long pass filter was used for the photolysis experiments, and a $400 \mathrm{~nm}$ bandpass filter and a $335 \mathrm{~nm}$ bandpass filter were used together for the ligand exchange quantum yield experiments. The samples were dissolved in $\mathrm{CH}_{2} \mathrm{Cl}_{2}$ with $10 \mathrm{mM} \mathrm{Bu}_{4} \mathrm{NCl}$ for each experiment. Potassium tris(ferrioxalate) was used as the chemical actinometer to determine the photon flux of the lamp with $\lambda_{\text {irr }}=400 \mathrm{~nm}\left(3.0 \times 10^{-8} \mathrm{~mol}\right.$ photons $\left./ \mathrm{min}\right) .{ }^{15}$ The rate of consumption of the reactant as a function of irradiation time ( $\mathrm{mol} \mathrm{reactant} / \mathrm{min})$ was determined by monitoring 
the decrease in absorbance at the MLCT $\lambda_{\max }$ and converting absorbance to moles using the extinction coefficient and sample volume. The quantum yield of ligand exchange was determined by dividing the rate of consumption of moles of reactant by the photon flux of the lamp. The reported values are an average of three measurements for each complex.

Stability of 1, 2 and 3 in DMSO and cell growth media (pH 7.2 Dulbecco's Modified Eagle's Medium).

\begin{tabular}{|c|c|c|c|}
\hline & $\mathbf{1}$ & $\mathbf{2}$ & $\mathbf{3}$ \\
\hline Concentration $(\mathrm{mM})$ & 2.38 & 1.99 & 2.35 \\
\hline
\end{tabular}

Stock solutions of complexes 1, 2 or $\mathbf{3}$ were prepared in DMSO at the concentrations shown above.

(1) Assays in $100 \%$ DMSO.

Take $4 \mu \mathrm{l}$ of stock solution $\mathbf{1}$ or $\mathbf{3}$ and $8 \mu \mathrm{l}$ of stock solution $\mathbf{2}$, and dilute with $200 \mu \mathrm{l}$ DMSO solvent, respectively. The assays were monitored by UV-Vis spectroscopy for $24 \mathrm{~h}$ at $23.0^{\circ} \mathrm{C}$. All the complexes were shown stable in the dark.

(2) Assays in cell growth media ( $<5 \%$ DMSO).

Take $4 \mu \mathrm{l}$ of stock solution 1 or 3 and $8 \mu$ l of stock solution 2 , and dilute with $200 \mu \mathrm{l}$ cell growth media, respectively. The assays were monitored by UV-Vis spectroscopy for $24 \mathrm{~h}$ at $37.0^{\circ} \mathrm{C}$. All the complexes were shown stable in the dark.

Cytotoxicity of 1,2 and 3 on prostate cancer PC-3 cells. PC-3 cell line derived from a bone metastasis of a high-grade adenocarcinoma ${ }^{16}$ was purchased from American Type Culture Collection (Manassas, VA) and cultured in DMEM media supplied with 10\% FBS. Cell viability under the dark and light conditions was measured using the MTT assay according to the manufacturer's instructions (Life Technologies, Carlsbad, CA). Briefly, the cells were grown in a 96-well plate for $24 \mathrm{~h}$. Cells were then treated with inhibitor or vehicle (1\% DMSO in normal growth media) for $30 \mathrm{~min}$, and the "dark" plate was wrapped in aluminum foil while the "light" plate was exposed to light. The photolysis was conducted for $40 \min \left(\lambda_{\text {irr }}>395 \mathrm{~nm}\right)$ and cells were placed in $37^{\circ} \mathrm{C}$ incubator under $5 \% \mathrm{CO}_{2}$ atmosphere. After $48 \mathrm{~h}$, the MTT assay was performed as described previously ${ }^{17}$ and absorbance at 540 nM was measured using Tecan Infinite 200 Pro Microplate Reader. Cell viabilities were calculated relative to the vehicle control and shown as mean +/- SEM. 


\section{Part C. X-ray Crystallographic Analysis}

\section{X-ray Crystallographic Analysis of $1\left[\mathrm{Ru}(\mathrm{TPA})(\mathrm{py})_{2}\right]\left(\mathrm{PF}_{6}\right)_{2}$.}

A specimen of $\mathrm{C}_{28} \mathrm{H}_{28} \mathrm{~F}_{12} \mathrm{~N}_{6} \mathrm{P}_{2} \mathrm{Ru}$ was used for the $\mathrm{X}$-ray crystallographic analysis. The $\mathrm{X}$-ray intensity data were measured.

The total exposure time was 14.93 hours. The frames were integrated with the Bruker SAINT software package using a narrow-frame algorithm. The integration of the data using a monoclinic unit cell yielded a total of 128259 reflections to a maximum $\theta$ angle of $28.02^{\circ}$ $(0.76 \AA$ resolution), of which 7659 were independent (average redundancy 16.746, completeness $\left.=99.5 \%, \mathrm{R}_{\text {int }}=4.01 \%\right)$ and $6649(86.81 \%)$ were greater than $2 \sigma\left(\mathrm{F}^{2}\right)$. The final cell constants of $\underline{a}=17.5692(11) \AA, \underline{b}=9.9426(6) \AA, \underline{c}=18.2432(12) \AA, \beta=93.013(4)^{\circ}$, volume $=3182.4(3) \AA^{3}$, are based upon the refinement of the XYZ-centroids of 9864 reflections above $20 \sigma(\mathrm{I})$ with $4.471^{\circ}<2 \theta<55.66^{\circ}$. Data were corrected for absorption effects using the multi-scan method (SADABS). The ratio of minimum to maximum $\begin{array}{llll}\text { apparent } & \text { transmission } & \text { was }\end{array}$

The final anisotropic full-matrix least-squares refinement on $\mathrm{F}^{2}$ with 442 variables converged at $\mathrm{R} 1=2.82 \%$, for the observed data and $\mathrm{wR} 2=6.44 \%$ for all data. The goodness-of-fit was 1.023. The largest peak in the final difference electron density synthesis was $0.899 \mathrm{e}^{\mathrm{e}} / \AA^{3}$ and the largest hole was $-0.886 \mathrm{e}^{-} / \AA^{3}$ with an RMS deviation of $0.070 \mathrm{e}^{-} / \AA^{3}$. On the basis of the final model, the calculated density was $1.752 \mathrm{~g} / \mathrm{cm}^{3}$ and $\mathrm{F}(000), 1680 \mathrm{e}$.

Table S1. Sample and crystal data for $\left[\mathrm{Ru}(\mathrm{TPA})(\mathrm{py})_{2}\right]\left(\mathrm{PF}_{6}\right)_{2}$.

Identification code

Chemical formula

Formula weight

Temperature

Wavelength

Crystal system

Space group

Unit cell dimensions ao95

$\mathrm{C}_{28} \mathrm{H}_{28} \mathrm{~F}_{12} \mathrm{~N}_{6} \mathrm{P}_{2} \mathrm{Ru}$

839.57

100(2) K

$0.71073 \AA$

monoclinic

P 1 21/c 1

$\mathrm{a}=17.5692(11) \AA$

$\mathrm{b}=9.9426(6) \AA$

$$
\begin{aligned}
& \alpha=90^{\circ} \\
& \beta=93.013(4)^{\circ}
\end{aligned}
$$


Volume

Z

Density (calculated)

Absorption coefficient

$\mathrm{F}(000)$ $\mathrm{c}=18.2432(12) \AA$

$3182.4(3) \AA^{3}$

4

$1.752 \mathrm{~g} / \mathrm{cm}^{3}$

$0.696 \mathrm{~mm}^{-1}$

1680

Table S2. Data collection and structure refinement for $\left[\mathrm{Ru}(\mathrm{TPA})(\mathrm{py})_{2}\right]\left(\mathrm{PF}_{6}\right)_{2}$.

$\begin{array}{ll}\text { Theta range for data collection } & 1.16 \text { to } 28.02^{\circ} \\ \text { Index ranges } & -23<=\mathrm{h}<=23,-13<=\mathrm{k}<=13,-23<=\mathrm{l}<=23 \\ \text { Reflections collected } & 128259 \\ \text { Independent reflections } & 7659[\mathrm{R}(\mathrm{int})=0.0401] \\ \text { Coverage of independent reflections } & 99.5 \% \\ \text { Absorption correction } & \text { multi-scan } \\ \text { Refinement method } & \text { Full-matrix least-squares on } \mathrm{F}^{2} \\ \text { Refinement program } & \mathrm{SHELXL}-2014 / 7(\text { Sheldrick, 2014) } \\ \text { Function minimized } & \sum \mathrm{w}\left(\mathrm{F}_{\mathrm{o}}{ }^{2}-\mathrm{F}_{\mathrm{c}}{ }^{2}\right)^{2} \\ \text { Data / restraints / parameters } & 7659 / 0 / 442 \\ \text { Goodness-of-fit on } \mathrm{F}^{2} & 1.023 \\ \Delta / \sigma_{\text {max }} & 0.001 \\ \text { Final R indices } & 6649 \text { data; } \mathrm{I}>2 \sigma(\mathrm{I}) \quad \mathrm{R} 1=0.0282, \mathrm{wR} 2=0.0606 \\ & \text { all data } \quad \mathrm{R} 1=0.0359, \mathrm{wR} 2=0.0644 \\ \text { Weighting scheme } & \mathrm{w}=1 /\left[\sigma^{2}\left(\mathrm{~F}_{\mathrm{o}}{ }^{2}\right)+(0.0233 \mathrm{P})^{2}+4.1772 \mathrm{P}\right] \\ \text { Largest diff. peak and hole } & \text { where } \mathrm{P}=\left(\mathrm{F}_{\mathrm{o}}{ }^{2}+2 \mathrm{~F}_{\mathrm{c}}{ }^{2}\right) / 3 \\ \text { R.M.S. deviation from mean } & 0.899 \text { and }-0.886 \mathrm{e} \AA^{-3} \\ & 0.070 \mathrm{e} \AA^{-3}\end{array}$

Table S3. Atomic coordinates and equivalent isotropic atomic displacement parameters $\left(\AA^{2}\right)$ for $\left[\mathrm{Ru}(\mathrm{TPA})(\mathrm{py})_{2}\right]\left(\mathrm{PF}_{6}\right)_{2}$.

$\mathrm{U}(\mathrm{eq})$ is defined as one third of the trace of the orthogonalized $\mathrm{U}_{\mathrm{ij}}$ tensor.

\begin{tabular}{lllll}
\hline & $\mathrm{x} / \mathrm{a}$ & $\mathrm{y} / \mathrm{b}$ & $\mathrm{z} / \mathrm{c}$ & $\mathrm{U}(\mathrm{eq})$ \\
$\mathrm{Ru} 1$ & $0.25936(2)$ & $0.21241(2)$ & $0.26985(2)$ & $0.01314(5)$ \\
$\mathrm{P} 1$ & $0.09058(3)$ & $0.69647(6)$ & $0.37510(3)$ & $0.02214(11)$ \\
$\mathrm{P} 2$ & $0.60685(3)$ & $0.16972(6)$ & $0.43082(3)$ & $0.02275(11)$ \\
$\mathrm{F} 1$ & $0.09387(8)$ & $0.84656(16)$ & $0.34489(9)$ & $0.0487(4)$
\end{tabular}




\begin{tabular}{|c|c|c|c|c|}
\hline & $\mathrm{x} / \mathrm{a}$ & $y / b$ & $\mathrm{z} / \mathrm{c}$ & $\mathrm{U}(\mathrm{eq})$ \\
\hline $\mathrm{F} 2$ & $0.02416(10)$ & $0.66701(17)$ & $0.31488(10)$ & $0.0607(5)$ \\
\hline $\mathrm{F} 3$ & $0.15171(12)$ & $0.6528(3)$ & $0.31942(9)$ & $0.1053(10)$ \\
\hline $\mathrm{F} 4$ & $0.08685(11)$ & $0.54773(15)$ & $0.40584(11)$ & $0.0671(6)$ \\
\hline F5 & $0.03051(10)$ & $0.74209(18)$ & $0.43086(9)$ & $0.0566(5)$ \\
\hline F6 & $0.15778(9)$ & $0.72662(16)$ & $0.43455(8)$ & $0.0443(4)$ \\
\hline F7 & $0.54577(8)$ & $0.28471(15)$ & $0.41212(9)$ & $0.0449(4)$ \\
\hline F8 & $0.53965(7)$ & $0.06268(14)$ & $0.43672(8)$ & $0.0398(3)$ \\
\hline F9 & $0.67400(8)$ & $0.27602(15)$ & $0.42538(8)$ & $0.0413(4)$ \\
\hline $\mathrm{F} 10$ & $0.66753(7)$ & $0.05306(14)$ & $0.44975(7)$ & $0.0333(3)$ \\
\hline $\mathrm{F} 11$ & $0.61149(8)$ & $0.13655(15)$ & $0.34518(7)$ & $0.0394(3)$ \\
\hline $\mathrm{F} 12$ & $0.60260(8)$ & $0.20102(15)$ & $0.51700(7)$ & $0.0388(3)$ \\
\hline N3 & $0.31709(9)$ & $0.05878(16)$ & $0.32657(8)$ & $0.0177(3)$ \\
\hline N5 & $0.31078(9)$ & $0.32725(16)$ & $0.35292(8)$ & $0.0160(3)$ \\
\hline N4 & $0.18117(9)$ & $0.18116(16)$ & $0.34939(8)$ & $0.0153(3)$ \\
\hline N2 & $0.35898(9)$ & $0.19734(16)$ & $0.21001(8)$ & $0.0159(3)$ \\
\hline N1 & $0.20067(9)$ & $0.37196(16)$ & $0.21511(8)$ & $0.0150(3)$ \\
\hline N6 & $0.20143(9)$ & $0.08845(16)$ & $0.19152(8)$ & $0.0157(3)$ \\
\hline $\mathrm{C} 1$ & $0.37712(11)$ & $0.1183(2)$ & $0.37865(10)$ & $0.0214(4)$ \\
\hline $\mathrm{C} 2$ & $0.35697(11)$ & $0.2580(2)$ & $0.40080(10)$ & $0.0192(4)$ \\
\hline $\mathrm{C} 3$ & $0.29095(11)$ & $0.4537(2)$ & $0.37089(10)$ & $0.0191(4)$ \\
\hline $\mathrm{C} 4$ & $0.31823(11)$ & $0.5161(2)$ & $0.43467(11)$ & $0.0223(4)$ \\
\hline C5 & $0.36754(12)$ & $0.4458(2)$ & $0.48267(11)$ & $0.0268(5)$ \\
\hline C6 & $0.38618(11)$ & $0.3151(2)$ & $0.46571(11)$ & $0.0250(4)$ \\
\hline $\mathrm{C} 7$ & $0.25962(11)$ & $0.9810(2)$ & $0.36643(11)$ & $0.0220(4)$ \\
\hline $\mathrm{C} 8$ & $0.19999(11)$ & $0.0745(2)$ & $0.39347(10)$ & $0.0190(4)$ \\
\hline C9 & $0.12613(10)$ & $0.26492(19)$ & $0.36987(10)$ & $0.0174(4)$ \\
\hline $\mathrm{C} 10$ & $0.08676(11)$ & $0.2446(2)$ & $0.43282(11)$ & $0.0220(4)$ \\
\hline $\mathrm{C} 11$ & $0.10577(12)$ & $0.1361(2)$ & $0.47705(11)$ & $0.0270(5)$ \\
\hline $\mathrm{C} 12$ & $0.16379(12)$ & $0.0509(2)$ & $0.45768(11)$ & $0.0261(4)$ \\
\hline C13 & $0.35473(11)$ & $0.9788(2)$ & $0.26971(11)$ & $0.0209(4)$ \\
\hline $\mathrm{C} 14$ & $0.39461(11)$ & $0.0768(2)$ & $0.22147(10)$ & $0.0192(4)$ \\
\hline $\mathrm{C} 15$ & $0.39395(10)$ & $0.2886(2)$ & $0.16906(10)$ & $0.0179(4)$ \\
\hline $\mathrm{C} 16$ & $0.46377(11)$ & $0.2658(2)$ & $0.13943(11)$ & $0.0218(4)$ \\
\hline $\mathrm{C} 17$ & $0.49883(11)$ & $0.1427(2)$ & $0.15041(11)$ & $0.0260(4)$ \\
\hline $\mathrm{C} 18$ & $0.46378(12)$ & $0.0472(2)$ & $0.19227(11)$ & $0.0243(4)$ \\
\hline C19 & $0.23407(11)$ & $0.48847(19)$ & $0.19618(10)$ & $0.0177(4)$ \\
\hline $\mathrm{C} 20$ & 0.19599(11) & $0.5920(2)$ & $0.15995(10)$ & $0.0205(4)$ \\
\hline
\end{tabular}




$\begin{array}{lllll} & \mathrm{x} / \mathrm{a} & \mathrm{y} / \mathrm{b} & \mathrm{z} / \mathrm{c} & \mathrm{U}(\mathrm{eq}) \\ \mathrm{C} 21 & 0.11883(12) & 0.5786(2) & 0.14250(11) & 0.0224(4) \\ \mathrm{C} 22 & 0.08276(11) & 0.4615(2) & 0.16230(10) & 0.0208(4) \\ \mathrm{C} 23 & 0.12492(10) & 0.3609(2) & 0.19723(10) & 0.0174(4) \\ \mathrm{C} 24 & 0.14361(11) & 0.0079(2) & 0.20916(11) & 0.0210(4) \\ \mathrm{C} 25 & 0.09990(12) & 0.9349(2) & 0.15839(12) & 0.0266(5) \\ \mathrm{C} 26 & 0.11531(12) & 0.9438(2) & 0.08504(12) & 0.0276(5) \\ \mathrm{C} 27 & 0.17424(11) & 0.0260(2) & 0.06581(11) & 0.0239(4) \\ \mathrm{C} 28 & 0.21545(11) & 0.0964(2) & 0.11946(10) & 0.0198(4)\end{array}$

Table S4. Bond lengths $(\AA)$ for $\left[\mathrm{Ru}(\mathrm{TPA})(\mathrm{py})_{2}\right]\left(\mathrm{PF}_{6}\right)_{2}$.

\begin{tabular}{llll}
\hline Ru1-N5 & $2.0670(16)$ & Ru1-N4 & $2.0734(15)$ \\
Ru1-N3 & $2.0791(16)$ & Ru1-N6 & $2.1081(16)$ \\
Ru1-N1 & $2.1139(15)$ & Ru1-N2 & $2.1162(15)$ \\
P1-F5 & $1.5707(14)$ & P1-F3 & $1.5775(16)$ \\
P1-F4 & $1.5842(17)$ & P1-F2 & $1.5874(16)$ \\
P1-F6 & $1.5893(15)$ & P1-F1 & $1.5929(15)$ \\
P2-F9 & $1.5910(14)$ & P2-F7 & $1.5927(14)$ \\
P2-F8 & $1.5973(14)$ & P2-F10 & $1.6007(14)$ \\
P2-F11 & $1.6031(14)$ & P2-F12 & $1.6083(14)$ \\
N3-C13 & $1.489(2)$ & N3-C7 & $1.491(2)$ \\
N3-C1 & $1.503(2)$ & N5-C2 & $1.349(2)$ \\
N5-C3 & $1.350(3)$ & N4-C9 & $1.344(2)$ \\
N4-C8 & $1.361(2)$ & N2-C15 & $1.344(2)$ \\
N2-C14 & $1.363(2)$ & N1-C19 & $1.352(2)$ \\
N1-C23 & $1.358(2)$ & N6-C24 & $1.346(2)$ \\
N6-C28 & $1.352(2)$ & C1-C2 & $1.494(3)$ \\
C1-H2 & 0.99 & C1-H1 & 0.99 \\
C2-C6 & $1.387(3)$ & C3-C4 & $1.382(3)$ \\
C3-H6 & 0.95 & C4-C5 & $1.388(3)$ \\
C4-H3 & 0.95 & C5-C6 & $1.379(3)$ \\
C5-H5 & 0.95 & C6-H4 & 0.95 \\
C7-C8 & $1.503(3)$ & C7-H11 & 0.99 \\
C7-H12 & 0.99 & C8-C12 & $1.382(3)$ \\
C9-C10 & $1.386(3)$ & C9-H10 & 0.95 \\
C10-C11 & $1.378(3)$ & C10-H9 & 0.95 \\
& & &
\end{tabular}




$\begin{array}{llll}\mathrm{C} 11-\mathrm{C} 12 & 1.386(3) & \mathrm{C} 11-\mathrm{H} 8 & 0.95 \\ \mathrm{C} 12-\mathrm{H} 7 & 0.95 & \mathrm{C} 13-\mathrm{C} 14 & 1.510(3) \\ \mathrm{C} 13-\mathrm{H} 13 & 0.99 & \mathrm{C} 13-\mathrm{H} 18 & 0.99 \\ \mathrm{C} 14-\mathrm{C} 18 & 1.384(3) & \mathrm{C} 15-\mathrm{C} 16 & 1.385(3) \\ \mathrm{C} 15-\mathrm{H} 17 & 0.95 & \mathrm{C} 16-\mathrm{C} 17 & 1.380(3) \\ \mathrm{C} 16-\mathrm{H} 16 & 0.95 & \mathrm{C} 17-\mathrm{C} 18 & 1.383(3) \\ \mathrm{C} 17-\mathrm{H} 15 & 0.95 & \mathrm{C} 18-\mathrm{H} 14 & 0.95 \\ \mathrm{C} 19-\mathrm{C} 20 & 1.377(3) & \mathrm{C} 19-\mathrm{H} 23 & 0.95 \\ \mathrm{C} 20-\mathrm{C} 21 & 1.382(3) & \mathrm{C} 20-\mathrm{H} 22 & 0.95 \\ \mathrm{C} 21-\mathrm{C} 22 & 1.383(3) & \mathrm{C} 21-\mathrm{H} 21 & 0.95 \\ \mathrm{C} 22-\mathrm{C} 23 & 1.381(3) & \mathrm{C} 22-\mathrm{H} 20 & 0.95 \\ \mathrm{C} 23-\mathrm{H} 19 & 0.95 & \mathrm{C} 24-\mathrm{C} 25 & 1.378(3) \\ \mathrm{C} 24-\mathrm{H} 28 & 0.95 & \mathrm{C} 25-\mathrm{C} 26 & 1.382(3) \\ \mathrm{C} 25-\mathrm{H} 27 & 0.95 & \mathrm{C} 26-\mathrm{C} 27 & 1.379(3) \\ \mathrm{C} 26-\mathrm{H} 26 & 0.95 & \mathrm{C} 27-\mathrm{C} 28 & 1.377(3) \\ \mathrm{C} 27-\mathrm{H} 25 & 0.95 & \mathrm{C} 28-\mathrm{H} 24 & 0.95\end{array}$

Table S5. Bond angles $\left(^{\circ}\right)$ for $\left[\mathrm{Ru}(\mathrm{TPA})(\mathrm{py})_{2}\right]\left(\mathrm{PF}_{6}\right)_{2}$.

\begin{tabular}{llll}
\hline N5-Ru1-N4 & $81.36(6)$ & N5-Ru1-N3 & $81.83(6)$ \\
N4-Ru1-N3 & $82.26(6)$ & N5-Ru1-N6 & $175.40(6)$ \\
N4-Ru1-N6 & $94.18(6)$ & N3-Ru1-N6 & $96.51(6)$ \\
N5-Ru1-N1 & $96.63(6)$ & N4-Ru1-N1 & $96.80(6)$ \\
N3-Ru1-N1 & $178.29(6)$ & N6-Ru1-N1 & $84.97(6)$ \\
N5-Ru1-N2 & $94.35(6)$ & N4-Ru1-N2 & $161.22(6)$ \\
N3-Ru1-N2 & $79.02(6)$ & N6-Ru1-N2 & $89.53(6)$ \\
N1-Ru1-N2 & $101.87(6)$ & F5-P1-F3 & $179.05(15)$ \\
F5-P1-F4 & $89.92(11)$ & F3-P1-F4 & $90.76(14)$ \\
F5-P1-F2 & $90.41(11)$ & F3-P1-F2 & $90.24(11)$ \\
F4-P1-F2 & $91.73(9)$ & F5-P1-F6 & $90.22(10)$ \\
F3-P1-F6 & $89.13(11)$ & F4-P1-F6 & $88.71(9)$ \\
F2-P1-F6 & $179.23(11)$ & F5-P1-F1 & $89.51(9)$ \\
F3-P1-F1 & $89.81(13)$ & F4-P1-F1 & $179.41(12)$ \\
F2-P1-F1 & $88.41(9)$ & F6-P1-F1 & $91.16(8)$ \\
F9-P2-F7 & $90.19(9)$ & F9-P2-F8 & $179.67(10)$ \\
F7-P2-F8 & $90.03(8)$ & F9-P2-F10 & $90.41(8)$ \\
F7-P2-F10 & $179.40(9)$ & F8-P2-F10 & $89.37(8)$
\end{tabular}




\begin{tabular}{|c|c|c|c|}
\hline F9-P2-F11 & 89.99(8) & F7-P2-F11 & $90.41(8)$ \\
\hline F8-P2-F11 & $90.25(8)$ & F10-P2-F11 & $89.64(7)$ \\
\hline F9-P2-F12 & $90.29(8)$ & F7-P2-F12 & $90.30(8)$ \\
\hline F8-P2-F12 & $89.46(8)$ & F10-P2-F12 & $89.65(8)$ \\
\hline F11-P2-F12 & 179.24(9) & C13-N3-C7 & $113.55(16)$ \\
\hline C13-N3-C1 & $109.14(15)$ & C7-N3-C1 & $111.51(15)$ \\
\hline C13-N3-Ru1 & $105.57(11)$ & C7-N3-Ru1 & $107.37(11)$ \\
\hline C1-N3-Ru1 & $109.46(12)$ & C2-N5-C3 & $118.17(17)$ \\
\hline C2-N5-Ru1 & $114.68(13)$ & C3-N5-Ru1 & $125.75(13)$ \\
\hline C9-N4-C8 & 118.33(16) & C9-N4-Ru1 & $127.92(13)$ \\
\hline C8-N4-Ru1 & $112.40(12)$ & C15-N2-C14 & $117.31(16)$ \\
\hline C15-N2-Ru1 & $130.81(13)$ & C14-N2-Ru1 & $111.65(12)$ \\
\hline C19-N1-C23 & $116.20(16)$ & C19-N1-Ru1 & $123.80(12)$ \\
\hline C23-N1-Ru1 & $120.00(12)$ & C24-N6-C28 & $116.46(16)$ \\
\hline C24-N6-Ru1 & $121.96(13)$ & C28-N6-Ru1 & $121.25(13)$ \\
\hline $\mathrm{C} 2-\mathrm{C} 1-\mathrm{N} 3$ & $111.62(15)$ & $\mathrm{C} 2-\mathrm{C} 1-\mathrm{H} 2$ & 109.3 \\
\hline N3-C1-H2 & 109.3 & C2-C1-H1 & 109.3 \\
\hline N3-C1-H1 & 109.3 & $\mathrm{H} 2-\mathrm{C} 1-\mathrm{H} 1$ & 108.0 \\
\hline N5-C2-C6 & 121.79(19) & $\mathrm{N} 5-\mathrm{C} 2-\mathrm{C} 1$ & $116.24(17)$ \\
\hline C6-C2-C1 & $121.94(18)$ & $\mathrm{N} 5-\mathrm{C} 3-\mathrm{C} 4$ & $122.70(19)$ \\
\hline N5-C3-H6 & 118.6 & C4-C3-H6 & 118.6 \\
\hline C3-C4-C5 & $118.8(2)$ & C3-C4-H3 & 120.6 \\
\hline C5-C4-H3 & 120.6 & C6-C5-C4 & $118.74(19)$ \\
\hline C6-C5-H5 & 120.6 & $\mathrm{C} 4-\mathrm{C} 5-\mathrm{H} 5$ & 120.6 \\
\hline C5-C6-C2 & $119.7(2)$ & C5-C6-H4 & 120.1 \\
\hline C2-C6-H4 & 120.1 & $\mathrm{~N} 3-\mathrm{C} 7-\mathrm{C} 8$ & $109.92(16)$ \\
\hline N3-C7-H11 & 109.7 & C8-C7-H11 & 109.7 \\
\hline N3-C7-H12 & 109.7 & C8-C7-H12 & 109.7 \\
\hline H11-C7-H12 & 108.2 & N4-C8-C12 & $121.66(18)$ \\
\hline $\mathrm{N} 4-\mathrm{C} 8-\mathrm{C} 7$ & $116.09(16)$ & C12-C8-C7 & $122.19(18)$ \\
\hline N4-C9-C10 & $122.53(18)$ & N4-C9-H10 & 118.7 \\
\hline C10-C9-H10 & 118.7 & C11-C10-C9 & $118.88(19)$ \\
\hline C11-C10-H9 & 120.6 & C9-C10-H9 & 120.6 \\
\hline C10-C11-C12 & 119.24(19) & C10-C11-H8 & 120.4 \\
\hline C12-C11-H8 & 120.4 & C8-C12-C11 & $119.32(19)$ \\
\hline
\end{tabular}




\begin{tabular}{|c|c|c|c|}
\hline C8-C12-H7 & 120.3 & $\mathrm{C} 11-\mathrm{C} 12-\mathrm{H} 7$ & 120.3 \\
\hline N3-C13-C14 & $107.35(16)$ & N3-C13-H13 & 110.2 \\
\hline C14-C13-H13 & 110.2 & N3-C13-H18 & 110.2 \\
\hline C14-C13-H18 & 110.2 & H13-C13-H18 & 108.5 \\
\hline N2-C14-C18 & $122.25(19)$ & N2-C14-C13 & $115.67(16)$ \\
\hline C18-C14-C13 & $122.07(18)$ & N2-C15-C16 & $123.13(19)$ \\
\hline N2-C15-H17 & 118.4 & $\mathrm{C} 16-\mathrm{C} 15-\mathrm{H} 17$ & 118.4 \\
\hline C17-C16-C15 & 119.11(19) & C17-C16-H16 & 120.4 \\
\hline C15-C16-H16 & 120.4 & C16-C17-C18 & $118.72(18)$ \\
\hline C16-C17-H15 & 120.6 & C18-C17-H15 & 120.6 \\
\hline C17-C18-C14 & $119.5(2)$ & $\mathrm{C} 17-\mathrm{C} 18-\mathrm{H} 14$ & 120.3 \\
\hline C14-C18-H14 & 120.3 & N1-C19-C20 & $123.81(18)$ \\
\hline N1-C19-H23 & 118.1 & $\mathrm{C} 20-\mathrm{C} 19-\mathrm{H} 23$ & 118.1 \\
\hline C19-C20-C21 & 118.97(19) & C19-C20-H22 & 120.5 \\
\hline $\mathrm{C} 21-\mathrm{C} 20-\mathrm{H} 22$ & 120.5 & $\mathrm{C} 20-\mathrm{C} 21-\mathrm{C} 22$ & $118.57(18)$ \\
\hline $\mathrm{C} 20-\mathrm{C} 21-\mathrm{H} 21$ & 120.7 & $\mathrm{C} 22-\mathrm{C} 21-\mathrm{H} 21$ & 120.7 \\
\hline C23-C22-C21 & $119.25(18)$ & $\mathrm{C} 23-\mathrm{C} 22-\mathrm{H} 20$ & 120.4 \\
\hline C21-C22-H20 & 120.4 & $\mathrm{~N} 1-\mathrm{C} 23-\mathrm{C} 22$ & $123.17(18)$ \\
\hline N1-C23-H19 & 118.4 & C22-C23-H19 & 118.4 \\
\hline N6-C24-C25 & 123.61(18) & N6-C24-H28 & 118.2 \\
\hline C25-C24-H28 & 118.2 & $\mathrm{C} 24-\mathrm{C} 25-\mathrm{C} 26$ & $119.10(19)$ \\
\hline C24-C25-H27 & 120.5 & $\mathrm{C} 26-\mathrm{C} 25-\mathrm{H} 27$ & 120.5 \\
\hline C27-C26-C25 & 118.19(19) & C27-C26-H26 & 120.9 \\
\hline C25-C26-H26 & 120.9 & $\mathrm{C} 28-\mathrm{C} 27-\mathrm{C} 26$ & 119.60(19) \\
\hline $\mathrm{C} 28-\mathrm{C} 27-\mathrm{H} 25$ & 120.2 & C26-C27-H25 & 120.2 \\
\hline N6-C28-C27 & $123.04(18)$ & N6-C28-H24 & 118.5 \\
\hline C27-C28-H24 & 118.5 & & \\
\hline
\end{tabular}

Table S6. Anisotropic atomic displacement parameters $\left(\AA^{2}\right)$ for $\left[\mathrm{Ru}(\mathrm{TPA})(\mathrm{py})_{2}\right]\left(\mathrm{PF}_{6}\right)_{2}$. 
The anisotropic atomic displacement factor exponent takes the form: $-2 \pi^{2}\left[h^{2} a^{* 2} U_{11}+\ldots+2\right.$ $\mathrm{h} \mathrm{k} \mathrm{a}{ }^{*} \mathrm{~b}^{*} \mathrm{U}_{12}$ ]

\begin{tabular}{|c|c|c|c|c|c|c|}
\hline & $\mathrm{U}_{11}$ & $\mathrm{U}_{22}$ & $\mathrm{U}_{33}$ & $\mathrm{U}_{23}$ & $\mathrm{U}_{13}$ & $\mathrm{U}_{12}$ \\
\hline $\mathrm{Ru} 1$ & $0.01299(7)$ & $0.01505(7)$ & $0.01146(7)$ & $0.00110(5)$ & $0.00156(5)$ & $0.00091(5)$ \\
\hline $\mathrm{P} 1$ & $0.0219(3)$ & $0.0254(3)$ & $0.0194(2)$ & $0.0000(2)$ & $0.0033(2)$ & $0.0071(2)$ \\
\hline $\mathrm{P} 2$ & $0.0187(2)$ & $0.0230(3)$ & $0.0267(3)$ & $-0.0037(2)$ & $0.0029(2)$ & $0.0008(2)$ \\
\hline $\mathrm{F} 1$ & $0.0353(8)$ & $0.0524(10)$ & $0.0575(10)$ & $0.0385(8)$ & $-0.0075(7)$ & $-0.0159(7)$ \\
\hline $\mathrm{F} 2$ & $0.0628(11)$ & $0.0411(9)$ & $0.0735(12)$ & $-0.0079(8)$ & $-0.0417(9)$ & $0.0077(8)$ \\
\hline $\mathrm{F} 3$ & $0.0895(15)$ & $0.204(3)$ & $0.0236(8)$ & $0.0027(12)$ & $0.0118(9)$ & $0.1097(18)$ \\
\hline $\mathrm{F} 4$ & $0.0884(14)$ & $0.0183(8)$ & $0.0899(14)$ & $0.0050(8)$ & $-0.0410(11)$ & $0.0061(8)$ \\
\hline F5 & $0.0598(10)$ & $0.0700(11)$ & $0.0436(9)$ & $0.0259(8)$ & $0.0355(8)$ & $0.0365(9)$ \\
\hline F6 & $0.0447(9)$ & $0.0465(9)$ & $0.0394(8)$ & $0.0118(7)$ & $-0.0183(7)$ & $-0.0065(7)$ \\
\hline F7 & $0.0360(8)$ & $0.0370(8)$ & $0.0628(10)$ & $0.0121(7)$ & $0.0145(7)$ & $0.0169(6)$ \\
\hline $\mathrm{F} 8$ & $0.0249(7)$ & $0.0346(8)$ & $0.0595(9)$ & $0.0013(7)$ & $-0.0024(6)$ & $-0.0080(6)$ \\
\hline F9 & $0.0340(7)$ & $0.0373(8)$ & $0.0545(9)$ & $-0.0151(7)$ & $0.0216(7)$ & $-0.0119(6)$ \\
\hline F10 & $0.0278(7)$ & $0.0399(8)$ & $0.0319(7)$ & $-0.0020(6)$ & $-0.0005(5)$ & $0.0125(6)$ \\
\hline $\mathrm{F} 11$ & $0.0514(9)$ & $0.0424(8)$ & $0.0239(7)$ & $-0.0046(6)$ & $-0.0034(6)$ & $0.0087(7)$ \\
\hline F12 & $0.0401(8)$ & $0.0457(9)$ & $0.0320(7)$ & $-0.0124(6)$ & $0.0139(6)$ & $-0.0020(6)$ \\
\hline N3 & $0.0180(8)$ & $0.0193(8)$ & $0.0161(8)$ & $0.0025(6)$ & $0.0024(6)$ & $0.0042(6)$ \\
\hline N5 & $0.0133(7)$ & $0.0216(8)$ & $0.0134(7)$ & $0.0007(6)$ & $0.0020(6)$ & $0.0000(6)$ \\
\hline N4 & $0.0155(7)$ & $0.0177(8)$ & $0.0129(7)$ & $0.0005(6)$ & $0.0026(6)$ & $-0.0017(6)$ \\
\hline $\mathrm{N} 2$ & $0.0139(7)$ & $0.0214(8)$ & $0.0124(7)$ & $-0.0007(6)$ & $0.0008(6)$ & $0.0011(6)$ \\
\hline N1 & $0.0162(7)$ & $0.0166(8)$ & $0.0123(7)$ & $0.0002(6)$ & $0.0022(6)$ & $0.0013(6)$ \\
\hline N6 & $0.0161(7)$ & $0.0153(7)$ & $0.0158(7)$ & $-0.0006(6)$ & $0.0018(6)$ & $0.0020(6)$ \\
\hline $\mathrm{C} 1$ & $0.0191(9)$ & $0.0282(11)$ & $0.0165(9)$ & $0.0027(8)$ & $-0.0014(7)$ & $0.0065(8)$ \\
\hline $\mathrm{C} 2$ & $0.0145(9)$ & $0.0269(10)$ & $0.0162(9)$ & $0.0014(8)$ & $0.0023(7)$ & $0.0015(7)$ \\
\hline $\mathrm{C} 3$ & $0.0164(9)$ & $0.0217(10)$ & $0.0194(9)$ & $0.0007(8)$ & $0.0030(7)$ & $-0.0019(7)$ \\
\hline $\mathrm{C} 4$ & $0.0202(10)$ & $0.0252(10)$ & $0.0219(10)$ & $-0.0052(8)$ & $0.0052(8)$ & $-0.0032(8)$ \\
\hline $\mathrm{C} 5$ & $0.0211(10)$ & $0.0408(13)$ & $0.0185(10)$ & $-0.0078(9)$ & $0.0004(8)$ & $-0.0016(9)$ \\
\hline C6 & $0.0188(10)$ & $0.0388(12)$ & $0.0173(9)$ & $-0.0019(9)$ & $-0.0007(7)$ & $0.0030(9)$ \\
\hline $\mathrm{C} 7$ & $0.0241(10)$ & $0.0202(10)$ & $0.0221(10)$ & $0.0071(8)$ & $0.0059(8)$ & $0.0038(8)$ \\
\hline $\mathrm{C} 8$ & $0.0210(9)$ & $0.0192(9)$ & $0.0170(9)$ & $0.0031(7)$ & $0.0026(7)$ & $0.0013(7)$ \\
\hline C9 & $0.0167(9)$ & $0.0181(9)$ & $0.0176(9)$ & $0.0009(7)$ & $0.0017(7)$ & $-0.0001(7)$ \\
\hline C10 & $0.0215(10)$ & $0.0263(10)$ & $0.0188(9)$ & $-0.0013(8)$ & $0.0069(8)$ & $0.0026(8)$ \\
\hline
\end{tabular}




$\begin{array}{lllllll} & \mathrm{U}_{11} & \mathrm{U}_{22} & \mathrm{U}_{33} & \mathrm{U}_{23} & \mathrm{U}_{13} & \mathrm{U}_{12} \\ \mathrm{C} 11 & 0.0306(11) & 0.0324(12) & 0.0189(10) & 0.0051(9) & 0.0103(8) & 0.0028(9) \\ \mathrm{C} 12 & 0.0318(11) & 0.0259(11) & 0.0213(10) & 0.0084(8) & 0.0074(8) & 0.0039(9) \\ \mathrm{C} 13 & 0.0223(10) & 0.0203(10) & 0.0203(9) & 0.0021(8) & 0.0034(8) & 0.0065(8) \\ \mathrm{C} 14 & 0.0191(9) & 0.0233(10) & 0.0152(9) & -0.0021(7) & 0.0007(7) & 0.0030(8) \\ \mathrm{C} 15 & 0.0169(9) & 0.0218(9) & 0.0150(9) & -0.0018(7) & 0.0010(7) & -0.0007(7) \\ \mathrm{C} 16 & 0.0159(9) & 0.0313(11) & 0.0182(9) & -0.0030(8) & 0.0017(7) & -0.0043(8) \\ \mathrm{C} 17 & 0.0168(9) & 0.0379(12) & 0.0237(10) & -0.0052(9) & 0.0043(8) & 0.0050(9) \\ \mathrm{C} 18 & 0.0225(10) & 0.0287(11) & 0.0217(10) & -0.0029(8) & 0.0027(8) & 0.0089(8) \\ \mathrm{C} 19 & 0.0161(9) & 0.0212(9) & 0.0162(9) & 0.0012(7) & 0.0048(7) & 0.0003(7) \\ \mathrm{C} 20 & 0.0265(10) & 0.0179(9) & 0.0177(9) & 0.0009(7) & 0.0062(8) & 0.0027(8) \\ \mathrm{C} 21 & 0.0275(10) & 0.0220(10) & 0.0175(9) & -0.0003(8) & -0.0005(8) & 0.0085(8) \\ \mathrm{C} 22 & 0.0187(9) & 0.0250(10) & 0.0184(9) & -0.0043(8) & -0.0022(7) & 0.0042(8) \\ \mathrm{C} 23 & 0.0186(9) & 0.0194(9) & 0.0144(9) & -0.0017(7) & 0.0013(7) & -0.0008(7) \\ \mathrm{C} 24 & 0.0261(10) & 0.0188(9) & 0.0188(9) & -0.0014(7) & 0.0063(8) & -0.0036(8) \\ \mathrm{C} 25 & 0.0292(11) & 0.0235(11) & 0.0277(11) & -0.0070(9) & 0.0082(9) & -0.0096(9) \\ \mathrm{C} 26 & 0.0275(11) & 0.0311(12) & 0.0245(11) & -0.0098(9) & 0.0030(8) & -0.0055(9) \\ \mathrm{C} 27 & 0.0223(10) & 0.0335(11) & 0.0163(9) & -0.0046(8) & 0.0033(8) & -0.0007(9) \\ \mathrm{C} 28 & 0.0180(9) & 0.0249(10) & 0.0166(9) & 0.0010(8) & 0.0029(7) & -0.0011(8)\end{array}$

Table S7. Hydrogen atomic coordinates and isotropic atomic displacement parameters $\left(\AA^{2}\right)$ for $\left[\mathrm{Ru}(\mathrm{TPA})(\mathrm{py})_{2}\right]\left(\mathrm{PF}_{6}\right)_{2}$.

\begin{tabular}{lllll}
\hline & $\mathrm{x} / \mathrm{a}$ & $\mathrm{y} / \mathrm{b}$ & $\mathrm{z} / \mathrm{c}$ & $\mathrm{U}(\mathrm{eq})$ \\
$\mathrm{H} 2$ & 0.3831 & 0.0610 & 0.4230 & 0.026 \\
$\mathrm{H} 1$ & 0.4265 & 0.1197 & 0.3549 & 0.026 \\
$\mathrm{H} 6$ & 0.2568 & 0.5018 & 0.3384 & 0.023 \\
$\mathrm{H} 3$ & 0.3035 & 0.6056 & 0.4455 & 0.027 \\
$\mathrm{H} 5$ & 0.3881 & 0.4869 & 0.5264 & 0.032 \\
$\mathrm{H} 4$ & 0.4189 & 0.2644 & 0.4983 & 0.03 \\
$\mathrm{H} 11$ & 0.2851 & -0.0668 & 0.4085 & 0.026 \\
$\mathrm{H} 12$ & 0.2353 & -0.0869 & 0.3332 & 0.026
\end{tabular}




$\begin{array}{lllll} & \mathrm{x} / \mathrm{a} & \mathrm{y} / \mathrm{b} & \mathrm{z} / \mathrm{c} & \mathrm{U}(\mathrm{eq}) \\ \text { H10 } & 0.1137 & 0.3410 & 0.3401 & 0.021 \\ \text { H9 } & 0.0473 & 0.3045 & 0.4453 & 0.026 \\ \text { H8 } & 0.0794 & 0.1199 & 0.5203 & 0.032 \\ \text { H7 } & 0.1785 & -0.0230 & 0.4882 & 0.031 \\ \text { H13 } & 0.3163 & -0.0736 & 0.2401 & 0.025 \\ \text { H18 } & 0.3920 & -0.0845 & 0.2932 & 0.025 \\ \text { H17 } & 0.3696 & 0.3727 & 0.1600 & 0.021 \\ \text { H16 } & 0.4872 & 0.3339 & 0.1119 & 0.026 \\ \text { H15 } & 0.5461 & 0.1239 & 0.1296 & 0.031 \\ \text { H14 } & 0.4870 & -0.0380 & 0.2009 & 0.029 \\ \text { H23 } & 0.2869 & 0.4996 & 0.2086 & 0.021 \\ \text { H22 } & 0.2223 & 0.6713 & 0.1471 & 0.025 \\ \text { H21 } & 0.0912 & 0.6483 & 0.1174 & 0.027 \\ \text { H20 } & 0.0296 & 0.4504 & 0.1520 & 0.025 \\ \text { H19 } & 0.0997 & 0.2799 & 0.2093 & 0.021 \\ \text { H28 } & 0.1323 & 0.0010 & 0.2594 & 0.025 \\ \text { H27 } & 0.0597 & -0.1208 & 0.1736 & 0.032 \\ \text { H26 } & 0.0861 & -0.1053 & 0.0489 & 0.033 \\ \text { H25 } & 0.1863 & 0.0341 & 0.0159 & 0.029 \\ \text { H24 } & 0.2556 & 0.1531 & 0.1052 & 0.024\end{array}$




\section{X-ray Crystallographic Analysis of $3\left[\operatorname{Ru}(\mathrm{TPA})(\mathrm{Im})_{2}\right]\left(\mathrm{PF}_{6}\right)_{2}$.}

A specimen of $\mathrm{C}_{24} \mathrm{H}_{26} \mathrm{~F}_{12} \mathrm{~N}_{6} \mathrm{P}_{2} \mathrm{Ru}$ was used for the $\mathrm{X}$-ray crystallographic analysis. The X-ray intensity data were measured.

The total exposure time was 7.79 hours. The frames were integrated with the Bruker SAINT software package using a narrow-frame algorithm. The integration of the data using a monoclinic unit cell yielded a total of 176881 reflections to a maximum $\theta$ angle of $25.77^{\circ}$ $(0.82 \AA$ resolution), of which 12892 were independent (average redundancy 13.720, completeness $\left.=99.6 \%, \mathrm{R}_{\text {int }}=4.21 \%\right)$ and $10612(82.31 \%)$ were greater than $2 \sigma\left(\mathrm{F}^{2}\right)$. The final cell constants of $\underline{a}=18.1540(12) \AA, \underline{b}=21.3364(12) \AA, \underline{c}=19.1704(12) \AA, \beta=114.733(3)^{\circ}$, volume $=6744.3(7) \AA^{3}$, are based upon the refinement of the XYZ-centroids of 9667 reflections above $20 \sigma(\mathrm{I})$ with $4.478^{\circ}<2 \theta<47.95^{\circ}$. Data were corrected for absorption effects using the multi-scan method (SADABS). The ratio of minimum to maximum apparent transmission was 0.892 .

The final anisotropic full-matrix least-squares refinement on $\mathrm{F}^{2}$ with 829 variables converged at $\mathrm{R} 1=11.22 \%$, for the observed data and $\mathrm{wR} 2=31.63 \%$ for all data. The goodness-of-fit was 1.045 . The largest peak in the final difference electron density synthesis was $2.788 \mathrm{e}^{-} / \AA^{3}$ and the largest hole was $-1.379 \mathrm{e}^{\mathrm{e}} / \AA^{3}$ with an RMS deviation of $0.219 \mathrm{e}^{\mathrm{e}} / \AA^{3}$. On the basis of the final model, the calculated density was $1.555 \mathrm{~g} / \mathrm{cm}^{3}$ and $\mathrm{F}(000), 3152 \mathrm{e}$.

This structure solution contains two crystallographically independent molecules in the asymmetric unit. One is well ordered and has good geometry and thermal ellipsoids. In particular, the imidazoles are stabilized by adjacent $\mathrm{PF}_{6}$ molecules, and the hydrophobic rings are packed closely together against symmetry related molecules.

The second molecule is severely disordered around one imidazole and one six membered ring to the point of not being modelable in the disordered area. This disorder has led to several red alerts, all related to these atoms. The first 7 red alerts are directly connected to and concerned with these atoms in the disordered molecule.

The final red alert, referring to the large solvent cavity, are probably due to highly disordered solvent molecules (trifluoroacetic acid and/or diethyl ether) to the point that they have virtually disappearing electron density. None of the residual density peaks is over 2.5 electrons and their positions do not affect the conclusions in this paper. We could have used SQUEEZE to model the cavities and lower the R-factor, but we are opposed to methods that modify the structure factors and choose to let the well ordered molecule stand on its own merits as presented in the paper. 
Table S8. Sample and crystal data for $\left[\mathrm{Ru}(\mathrm{TPA})(\text { imidazole })_{2}\right]\left(\mathrm{PF}_{6}\right)_{2}$.

\begin{tabular}{|c|c|c|}
\hline Identification code & ao02158 & \\
\hline Chemical formula & $\mathrm{C}_{24} \mathrm{H}_{26} \mathrm{~F}_{12} \mathrm{~N}_{6} \mathrm{P}_{2} \mathrm{Ru}$ & \\
\hline Formula weight & 789.52 & \\
\hline Temperature & $100(2) \mathrm{K}$ & \\
\hline Wavelength & $0.71073 \AA$ & \\
\hline Crystal system & monoclinic & \\
\hline Space group & P $121 / \mathrm{c} 1$ & \\
\hline \multirow[t]{3}{*}{ Unit cell dimensions } & $\mathrm{a}=18.1540(12) \AA$ & $\alpha=90^{\circ}$ \\
\hline & $\mathrm{b}=21.3364(12) \AA$ & $\beta=114.733(3)^{\circ}$ \\
\hline & $\mathrm{c}=19.1704(12) \AA$ & $\gamma=90^{\circ}$ \\
\hline Volume & $6744.3(7) \AA^{3}$ & \\
\hline $\mathrm{Z}$ & 8 & \\
\hline Density (calculated) & $1.555 \mathrm{~g} / \mathrm{cm}^{3}$ & \\
\hline Absorption coefficient & $0.651 \mathrm{~mm}^{-1}$ & \\
\hline $\mathrm{F}(000)$ & 3152 & \\
\hline
\end{tabular}

Table S9. Data collection and structure refinement for $\left[\mathrm{Ru}(\mathrm{TPA})(\text { imidazole })_{2}\right]\left(\mathrm{PF}_{6}\right)_{2}$.

\begin{tabular}{|c|c|c|}
\hline Theta range for data collection & \multicolumn{2}{|l|}{1.24 to $25.77^{\circ}$} \\
\hline Index ranges & \multicolumn{2}{|c|}{$-22<=\mathrm{h}<=22,-26<=\mathrm{k}<=25,-22<=\mathrm{l}<=23$} \\
\hline Reflections collected & \multicolumn{2}{|l|}{176881} \\
\hline Independent reflections & \multicolumn{2}{|c|}{$12892[\mathrm{R}(\mathrm{int})=0.0421]$} \\
\hline Coverage of independent reflections & \multicolumn{2}{|l|}{$99.6 \%$} \\
\hline Absorption correction & \multicolumn{2}{|l|}{ multi-scan } \\
\hline Refinement method & \multicolumn{2}{|c|}{ Full-matrix least-squares on $\mathrm{F}^{2}$} \\
\hline Refinement program & \multicolumn{2}{|c|}{ SHELXL-2014/7 (Sheldrick, 2014) } \\
\hline Function minimized & \multicolumn{2}{|l|}{$\sum \mathrm{w}\left(\mathrm{F}_{\mathrm{o}}^{2}-\mathrm{F}_{\mathrm{c}}^{2}\right)^{2}$} \\
\hline Data / restraints / parameters & \multicolumn{2}{|l|}{12892 / 0 / 829} \\
\hline Goodness-of-fit on $\mathrm{F}^{2}$ & \multicolumn{2}{|l|}{1.045} \\
\hline$\Delta / \sigma_{\max }$ & \multicolumn{2}{|l|}{0.591} \\
\hline \multirow[t]{2}{*}{ Final $\mathrm{R}$ indices } & 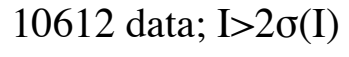 & $\mathrm{R} 1=0.1122, \mathrm{wR} 2=0.2984$ \\
\hline & all data & $\mathrm{R} 1=0.1298, w \mathrm{R} 2=0.3163$ \\
\hline Weighting scheme & \multicolumn{2}{|c|}{$\mathrm{w}=1 /\left[\sigma^{2}\left(\mathrm{~F}_{\mathrm{o}}^{2}\right)+(0.1630 \mathrm{P})^{2}+89.1685 \mathrm{P}\right]$} \\
\hline
\end{tabular}


where $\mathrm{P}=\left(\mathrm{F}_{\mathrm{o}}{ }^{2}+2 \mathrm{~F}_{\mathrm{c}}{ }^{2}\right) / 3$

Largest diff. peak and hole 2.788 and $-1.379 \mathrm{e}^{-3}$

R.M.S. deviation from mean

Table S10. Atomic coordinates and equivalent isotropic atomic displacement parameters $\left(\AA^{2}\right)$ for $\left.[\mathrm{Ru}(\mathrm{TPA}) \text { (imidazole })_{2}\right]\left(\mathrm{PF}_{6}\right)_{2}$.

$\mathrm{U}(\mathrm{eq})$ is defined as one third of the trace of the orthogonalized $\mathrm{U}_{\mathrm{ij}}$ tensor.

\begin{tabular}{|c|c|c|c|c|}
\hline & $\mathrm{x} / \mathrm{a}$ & $y / b$ & $\mathrm{z} / \mathrm{c}$ & $\mathrm{U}(\mathrm{eq})$ \\
\hline $\mathrm{Ru} 01$ & $0.22733(4)$ & $0.14312(3)$ & $0.30851(4)$ & $0.0326(2)$ \\
\hline $\mathrm{Ru} 02$ & $0.76498(4)$ & $0.37876(4)$ & $0.62130(4)$ & $0.0400(2)$ \\
\hline P003 & $0.51596(17)$ & $0.65203(16)$ & $0.73421(16)$ & $0.0592(9)$ \\
\hline P004 & $0.9008(2)$ & $0.59137(14)$ & $0.90964(18)$ & $0.0627(8)$ \\
\hline P005 & $0.8470(2)$ & $0.13917(18)$ & $0.8367(2)$ & $0.0669(9)$ \\
\hline P006 & $0.3562(5)$ & $0.36597(18)$ & $0.5517(2)$ & $0.114(2)$ \\
\hline F007 & $0.4830(5)$ & $0.6396(4)$ & $0.7977(4)$ & $0.084(3)$ \\
\hline F008 & $0.8598(5)$ & $0.1287(3)$ & $0.9239(4)$ & $0.0685(19)$ \\
\hline N3 & $0.2941(5)$ & $0.2117(3)$ & $0.2840(5)$ & $0.0392(17)$ \\
\hline NOOA & $0.8252(5)$ & $0.3074(3)$ & $0.5944(4)$ & $0.0383(16)$ \\
\hline N4 & $0.1646(4)$ & $0.2193(3)$ & $0.3213(4)$ & $0.0363(16)$ \\
\hline N2 & $0.3131(5)$ & $0.0858(3)$ & $0.2937(4)$ & $0.0376(16)$ \\
\hline N5 & $0.1548(5)$ & $0.1525(3)$ & $0.1919(4)$ & $0.0330(15)$ \\
\hline F00E & $0.4591(6)$ & $0.6003(4)$ & $0.6810(4)$ & $0.097(3)$ \\
\hline N6 & $0.3011(5)$ & $0.1401(4)$ & $0.4272(5)$ & $0.0437(18)$ \\
\hline F00G & $0.5504(5)$ & $0.6649(5)$ & $0.6725(5)$ & $0.107(4)$ \\
\hline $\mathrm{NOOH}$ & $0.8379(5)$ & $0.3815(3)$ & $0.7399(4)$ & $0.0388(17)$ \\
\hline N1 & $0.1591(5)$ & $0.0722(4)$ & $0.3322(5)$ & $0.0429(18)$ \\
\hline N00J & $0.8299(5)$ & $0.4415(3)$ & $0.5885(5)$ & $0.0410(17)$ \\
\hline F00K & $0.4479(7)$ & $0.7022(6)$ & $0.6937(5)$ & $0.121(4)$ \\
\hline NOOL & $0.6915(5)$ & $0.4515(4)$ & $0.6301(4)$ & $0.049(2)$ \\
\hline F00M & $0.8926(7)$ & $0.5269(4)$ & $0.9474(5)$ & $0.099(3)$ \\
\hline F00N & $0.9083(8)$ & $0.6561(4)$ & $0.8718(6)$ & $0.107(3)$ \\
\hline $\mathrm{COOO}$ & $0.0913(5)$ & $0.2208(4)$ & $0.3246(5)$ & $0.0378(19)$ \\
\hline F00P & $0.3089(7)$ & $0.3973(4)$ & $0.6039(6)$ & $0.106(3)$ \\
\hline CO0Q & $0.2001(6)$ & $0.2744(4)$ & $0.3182(5)$ & $0.041(2)$ \\
\hline F00R & $0.5841(6)$ & $0.6016(6)$ & $0.7756(6)$ & $0.133(5)$ \\
\hline
\end{tabular}




\begin{tabular}{|c|c|c|c|c|}
\hline & $\mathrm{x} / \mathrm{a}$ & $y / b$ & $\mathrm{z} / \mathrm{c}$ & U(eq) \\
\hline NOOS & $0.6936(5)$ & $0.3749(6)$ & $0.5032(5)$ & $0.067(3)$ \\
\hline C00T & $0.0546(6)$ & $0.2763(5)$ & $0.3288(6)$ & $0.048(2)$ \\
\hline COOU & $0.2663(7)$ & $0.2176(5)$ & $0.1988(6)$ & $0.051(3)$ \\
\hline $\mathrm{COOV}$ & $0.0308(7)$ & $0.1430(5)$ & $0.0783(6)$ & $0.051(2)$ \\
\hline NOW & $0.1037(6)$ & $0.0195(5)$ & $0.3960(6)$ & $0.065(3)$ \\
\hline NOOX & $0.6840(6)$ & $0.3156(5)$ & $0.6326(8)$ & $0.085(4)$ \\
\hline $\mathrm{COOY}$ & $0.0792(6)$ & $0.1287(4)$ & $0.1537(6)$ & $0.043(2)$ \\
\hline COOZ & $0.3110(6)$ & $0.0246(4)$ & $0.2773(5)$ & $0.042(2)$ \\
\hline $\mathrm{C} 010$ & $0.8970(6)$ & $0.2814(4)$ & $0.6400(6)$ & $0.043(2)$ \\
\hline C011 & $0.1814(6)$ & $0.1932(4)$ & $0.1549(6)$ & $0.041(2)$ \\
\hline $\mathrm{C} 012$ & $0.2829(6)$ & $0.2707(4)$ & $0.3188(6)$ & $0.047(2)$ \\
\hline N1A & $0.3548(7)$ & $0.1610(6)$ & $0.5497(6)$ & $0.075(3)$ \\
\hline $\mathrm{C} 013$ & $0.1640(6)$ & $0.3314(5)$ & $0.3186(6)$ & $0.048(2)$ \\
\hline $\mathrm{C} 014$ & $0.3789(6)$ & $0.1196(5)$ & $0.2995(6)$ & $0.046(2)$ \\
\hline F015 & $0.9360(7)$ & $0.1201(5)$ & $0.8585(10)$ & $0.157(6)$ \\
\hline F016 & $0.5757(8)$ & $0.7027(6)$ & $0.7880(5)$ & $0.172(7)$ \\
\hline $\mathrm{C} 017$ & $0.9034(6)$ & $0.4663(4)$ & $0.6297(7)$ & $0.047(2)$ \\
\hline C018 & $0.1443(6)$ & $0.0709(5)$ & $0.3949(6)$ & $0.050(2)$ \\
\hline C019 & $0.7936(7)$ & $0.4566(4)$ & $0.5128(6)$ & $0.052(3)$ \\
\hline F01B & $0.9393(11)$ & $0.6202(6)$ & $0.9865(6)$ & $0.176(7)$ \\
\hline $\mathrm{C} 01 \mathrm{C}$ & $0.3794(6)$ & $0.1878(5)$ & $0.3190(6)$ & $0.046(2)$ \\
\hline C01D & $0.0601(8)$ & $0.1848(5)$ & $0.0403(6)$ & $0.059(3)$ \\
\hline F01E & $0.8281(11)$ & $0.0668(7)$ & $0.8234(8)$ & $0.218(10)$ \\
\hline $\mathrm{C} 01 \mathrm{~F}$ & $0.8664(6)$ & $0.3315(5)$ & $0.7886(5)$ & $0.043(2)$ \\
\hline F01G & $0.8783(10)$ & $0.2073(4)$ & $0.8587(10)$ & $0.171(7)$ \\
\hline $\mathrm{C} 01 \mathrm{H}$ & $0.3740(6)$ & $0.9949(5)$ & $0.2680(6)$ & $0.048(2)$ \\
\hline C01I & $0.4438(7)$ & $0.0910(5)$ & $0.2915(7)$ & $0.055(3)$ \\
\hline $\mathrm{C} 01 \mathrm{~J}$ & $0.0919(7)$ & $0.3321(5)$ & $0.3259(6)$ & $0.054(3)$ \\
\hline $\mathrm{C} 01 \mathrm{~K}$ & $0.7900(9)$ & $0.2875(5)$ & $0.5221(6)$ & $0.067(4)$ \\
\hline $\mathrm{C} 01 \mathrm{~L}$ & $0.7112(8)$ & $0.4297(5)$ & $0.4665(6)$ & $0.057(3)$ \\
\hline $\mathrm{C} 01 \mathrm{M}$ & $0.1341(7)$ & $0.2101(5)$ & $0.0791(6)$ & $0.050(2)$ \\
\hline $\mathrm{C} 01 \mathrm{~N}$ & $0.9414(7)$ & $0.5069(5)$ & $0.6003(8)$ & $0.060(3)$ \\
\hline $\mathrm{C} 1 \mathrm{O}$ & $0.1261(6)$ & $0.0182(4)$ & $0.2939(7)$ & $0.050(2)$ \\
\hline N1P & $0.9057(8)$ & $0.4156(5)$ & $0.8554(6)$ & $0.082(4)$ \\
\hline C01Q & $0.4406(7)$ & $0.0281(6)$ & $0.2761(7)$ & $0.060(3)$ \\
\hline F01R & $0.9515(14)$ & $0.5620(7)$ & $0.8795(16)$ & $0.310(15)$ \\
\hline C01S & $0.7099(11)$ & $0.3156(6)$ & $0.4721(8)$ & $0.107(7)$ \\
\hline
\end{tabular}




$\begin{array}{lllll} & \mathrm{x} / \mathrm{a} & \mathrm{y} / \mathrm{b} & \mathrm{z} / \mathrm{c} & \mathrm{U}(\mathrm{eq}) \\ \mathrm{C} 01 \mathrm{~T} & 0.8287(9) & 0.4964(5) & 0.4791(7) & 0.063(3) \\ \mathrm{C} 01 \mathrm{U} & 0.9314(9) & 0.2343(5) & 0.6152(8) & 0.068(3) \\ \mathrm{C} 01 \mathrm{~V} & 0.3082(6) & 0.1825(6) & 0.4805(6) & 0.054(3) \\ \mathrm{C} 1 \mathrm{~W} & 0.0929(7) & 0.9848(5) & 0.3321(8) & 0.065(3) \\ \mathrm{C} 1 \mathrm{X} & 0.9095(7) & 0.3528(6) & 0.8611(6) & 0.057(3) \\ \mathrm{C} 01 \mathrm{Y} & 0.3458(9) & 0.0899(6) & 0.4656(6) & 0.066(3) \\ \text { F01Z } & 0.4236(15) & 0.3437(10) & 0.6326(9) & 0.270(14) \\ \text { C020 } & 0.9047(10) & 0.5229(5) & 0.5266(9) & 0.074(4) \\ \text { F021 } & 0.3789(15) & 0.3333(9) & 0.4956(11) & 0.306(15) \\ \text { F023 } & 0.2879(9) & 0.4089(8) & 0.4820(7) & 0.162(6) \\ \text { C24 } & 0.3790(10) & 0.1014(7) & 0.5426(7) & 0.083(4) \\ \text { C025 } & 0.8635(10) & 0.4309(5) & 0.7823(7) & 0.073(4) \\ \text { C028 } & 0.6858(10) & 0.2673(7) & 0.6733(9) & 0.091(5) \\ \text { F02C } & 0.3052(19) & 0.3121(5) & 0.5472(16) & 0.346(17) \\ \text { C02H } & 0.6205(12) & 0.2474(16) & 0.6764(11) & 0.200(16) \\ \text { F02I } & 0.8312(11) & 0.1561(11) & 0.7537(7) & 0.219(10) \\ \text { C02J } & 0.8930(15) & 0.2129(7) & 0.5416(10) & 0.116(8) \\ \text { N2M } & 0.6287(16) & 0.5422(8) & 0.6357(17) & 0.196(12) \\ \text { F02N } & 0.7601(7) & 0.1624(14) & 0.8206(7) & 0.281(15) \\ \text { C02O } & 0.6105(11) & 0.3102(10) & 0.5510(10) & 0.102(5) \\ \text { F02P } & 0.8234(8) & 0.5741(8) & 0.8319(9) & 0.163(6) \\ \text { C02Q } & 0.6094(9) & 0.381(2) & 0.4987(11) & 0.23(2) \\ \text { C02R } & 0.5449(10) & 0.245(2) & 0.574(3) & 0.32(3) \\ \text { C02S } & 0.6967(10) & 0.5042(6) & 0.645(2) & 0.23(2) \\ \text { C2V } & 0.6068(19) & 0.465(3) & 0.6517(11) & 0.32(3) \\ \text { F2 } & 0.8231(9) & 0.6162(7) & 0.9207(13) & 0.185(7) \\ \text { F6 } & 0.4161(12) & 0.4169(9) & 0.5644(11) & 0.254(12) \\ \text { C3 } & 0.8253(14) & 0.2394(7) & 0.4952(8) & 0.102(7)\end{array}$

Table S11. Bond lengths $(\AA)$ for $\left[\mathrm{Ru}(\mathrm{TPA})(\text { imidazole })_{2}\right]\left(\mathrm{PF}_{6}\right)_{2}$.

\begin{tabular}{llll}
\hline Ru01-N4 & $2.057(7)$ & Ru01-N5 & $2.076(7)$ \\
Ru01-N3 & $2.077(7)$ & Ru01-N2 & $2.091(7)$ \\
Ru01-N6 & $2.103(8)$ & Ru01-N1 & $2.121(7)$ \\
Ru02-N00J & $2.048(8)$ & Ru02-N00A & $2.063(8)$ \\
Ru02-N00X & $2.072(10)$ & Ru02-N00S & $2.088(9)$
\end{tabular}




\begin{tabular}{|c|c|c|c|}
\hline Ru02-NOOH & $2.099(8)$ & Ru02-N00L & $2.099(8)$ \\
\hline P003-F00E & $1.562(8)$ & P003-F00K & $1.572(10)$ \\
\hline P003-F016 & $1.573(9)$ & P003-F00G & $1.577(8)$ \\
\hline P003-F00R & $1.579(10)$ & P003-F007 & $1.588(8)$ \\
\hline P004-F01R & $1.420(10)$ & P004-F01B & $1.475(9)$ \\
\hline P004-F00M & $1.589(8)$ & P004-F2 & $1.601(16)$ \\
\hline P004-F00N & $1.593(9)$ & P004-F02P & $1.606(14)$ \\
\hline P005-F02I & $1.536(11)$ & P005-F015 & $1.545(10)$ \\
\hline P005-F01G & $1.554(12)$ & $\mathrm{P} 005-\mathrm{F} 02 \mathrm{~N}$ & $1.556(12)$ \\
\hline P005-F01E & $1.579(13)$ & P005-F008 & $1.604(8)$ \\
\hline P006-F02C & $1.456(14)$ & P006-F021 & $1.477(11)$ \\
\hline P006-F6 & $1.483(15)$ & P006-F01Z & $1.594(16)$ \\
\hline P006-F023 & $1.666(14)$ & P006-F00P & $1.705(11)$ \\
\hline N3-C012 & $1.477(11)$ & N3-C01C & $1.497(12)$ \\
\hline N3-C00U & $1.499(13)$ & N00A-C01K & $1.330(12)$ \\
\hline N00A-C010 & $1.347(12)$ & N4-C00Q & $1.355(12)$ \\
\hline N4-C00O & $1.358(11)$ & $\mathrm{N} 2-\mathrm{C} 00 \mathrm{Z}$ & $1.340(11)$ \\
\hline N2-C014 & $1.361(12)$ & N5-C011 & $1.332(12)$ \\
\hline N5-C00Y & $1.356(12)$ & N6-C01V & $1.332(12)$ \\
\hline N6-C01Y & $1.360(15)$ & NOOH-C025 & $1.293(14)$ \\
\hline N00H-C01F & $1.370(12)$ & $\mathrm{N} 1-\mathrm{C} 018$ & $1.337(12)$ \\
\hline $\mathrm{N} 1-\mathrm{C} 1 \mathrm{O}$ & $1.363(12)$ & N00J-C017 & $1.343(13)$ \\
\hline N00J-C019 & $1.358(13)$ & N00L-C02S & $1.155(18)$ \\
\hline N00L-C2V & $1.78(4)$ & $\mathrm{C} 00 \mathrm{O}-\mathrm{C} 00 \mathrm{~T}$ & $1.377(13)$ \\
\hline COOO-H00O & 0.95 & C00Q-C013 & $1.381(14)$ \\
\hline C00Q-C012 & $1.502(13)$ & N00S-C01L & $1.467(15)$ \\
\hline N00S-C01S & $1.48(2)$ & N00S-C02Q & $1.498(18)$ \\
\hline C00T-C01J & $1.382(15)$ & COOT-H0OT & 0.95 \\
\hline C00U-C011 & $1.505(14)$ & COOU-H0OA & 0.99 \\
\hline COOU-H00B & 0.99 & $\mathrm{COOV}-\mathrm{COOY}$ & $1.375(15)$ \\
\hline C00V-C01D & $1.390(16)$ & COOV-H0OV & 0.95 \\
\hline N0W-C018 & $1.326(14)$ & NoW-C1W & $1.373(17)$ \\
\hline NOW-HOW & 0.88 & N00X-C028 & $1.285(16)$ \\
\hline N00X-C02O & $1.58(2)$ & COOY-H0OY & 0.95 \\
\hline C00Z-C01H & $1.382(14)$ & COOZ-H0OZ & 0.95 \\
\hline C010-C01U & $1.370(14)$ & C010-H010 & 0.95 \\
\hline C011-C01M & $1.390(14)$ & C012-H01A & 0.99 \\
\hline C012-H01B & 0.99 & N1A-C01V & $1.322(16)$ \\
\hline
\end{tabular}




\begin{tabular}{|c|c|c|c|}
\hline $\mathrm{N} 1 \mathrm{~A}-\mathrm{C} 24$ & $1.371(18)$ & N1A-H01C & 0.88 \\
\hline $\mathrm{C} 013-\mathrm{C} 01 \mathrm{~J}$ & $1.375(15)$ & C013-H013 & 0.95 \\
\hline C014-C01I & $1.390(14)$ & C014-C01C & $1.500(13)$ \\
\hline $\mathrm{C} 017-\mathrm{C} 01 \mathrm{~N}$ & $1.366(15)$ & C017-H017 & 0.95 \\
\hline C018-H018 & 0.95 & C019-C01T & $1.374(16)$ \\
\hline C019-C01L & $1.498(17)$ & C01C-H01D & 0.99 \\
\hline C01C-H01E & 0.99 & C01D-C01M & $1.347(16)$ \\
\hline C01D-H01F & 0.95 & C01F-C1X & $1.356(15)$ \\
\hline C01F-H01G & 0.95 & $\mathrm{C} 01 \mathrm{H}-\mathrm{C} 01 \mathrm{Q}$ & $1.352(16)$ \\
\hline $\mathrm{C} 01 \mathrm{H}-\mathrm{H} 01 \mathrm{H}$ & 0.95 & C01I-C01Q & $1.371(15)$ \\
\hline C01I-H01I & 0.95 & C01J-H01J & 0.95 \\
\hline C01K-C3 & $1.42(2)$ & $\mathrm{C} 01 \mathrm{~K}-\mathrm{C} 01 \mathrm{~S}$ & $1.49(2)$ \\
\hline C01L-H01K & 0.99 & C01L-H01L & 0.99 \\
\hline C01M-H01M & 0.95 & $\mathrm{C} 01 \mathrm{~N}-\mathrm{C} 020$ & $1.330(19)$ \\
\hline C01N-H01N & 0.95 & C1O-C1W & $1.332(15)$ \\
\hline $\mathrm{C} 1 \mathrm{O}-\mathrm{H} 1 \mathrm{O}$ & 0.95 & N1P-C025 & $1.325(17)$ \\
\hline N1P-C1X & $1.345(16)$ & N1P-H1P & 0.88 \\
\hline C01Q-H01Q & 0.95 & C01S-H01R & 0.99 \\
\hline C01S-H01S & 0.99 & C01T-C020 & $1.41(2)$ \\
\hline C01T-H01T & 0.95 & C01U-C02J & $1.37(2)$ \\
\hline C01U-H01U & 0.95 & C01V-H01V & 0.95 \\
\hline $\mathrm{C} 1 \mathrm{~W}-\mathrm{H} 1 \mathrm{~W}$ & 0.95 & C1X-H1X & 0.95 \\
\hline $\mathrm{C} 01 \mathrm{Y}-\mathrm{C} 24$ & $1.362(16)$ & C01Y-H01Y & 0.95 \\
\hline $\mathrm{C} 020-\mathrm{H} 020$ & 0.95 & C24-H24 & 0.95 \\
\hline C025-H025 & 0.95 & $\mathrm{C} 028-\mathrm{C} 02 \mathrm{H}$ & $1.28(2)$ \\
\hline $\mathrm{C} 028-\mathrm{H} 028$ & 0.95 & $\mathrm{C} 02 \mathrm{H}-\mathrm{C} 02 \mathrm{R}$ & $1.86(4)$ \\
\hline $\mathrm{C} 02 \mathrm{H}-\mathrm{H} 02 \mathrm{H}$ & 0.95 & $\mathrm{C} 02 \mathrm{~J}-\mathrm{C} 3$ & $1.31(3)$ \\
\hline $\mathrm{C} 02 \mathrm{~J}-\mathrm{H} 02 \mathrm{~J}$ & 0.95 & $\mathrm{~N} 2 \mathrm{M}-\mathrm{C} 02 \mathrm{~S}$ & $1.42(2)$ \\
\hline $\mathrm{N} 2 \mathrm{M}-\mathrm{C} 2 \mathrm{~V}$ & $1.74(5)$ & $\mathrm{C} 02 \mathrm{O}-\mathrm{C} 02 \mathrm{Q}$ & $1.80(4)$ \\
\hline $\mathrm{C} 02 \mathrm{O}-\mathrm{C} 02 \mathrm{R}$ & $2.00(5)$ & $\mathrm{C} 02 \mathrm{~S}-\mathrm{C} 2 \mathrm{~V}$ & $1.88(3)$ \\
\hline $\mathrm{C} 3-\mathrm{H} 3$ & 0.95 & & \\
\hline
\end{tabular}

Table S12. Bond angles $\left({ }^{\circ}\right)$ for $\left[\mathrm{Ru}(\mathrm{TPA})(\text { imidazole })_{2}\right]\left(\mathrm{PF}_{6}\right)_{2}$.

\begin{tabular}{llll}
\hline N4-Ru01-N5 & $85.3(3)$ & N4-Ru01-N3 & $82.8(3)$ \\
N5-Ru01-N3 & $81.7(3)$ & N4-Ru01-N2 & $163.6(3)$ \\
N5-Ru01-N2 & $94.7(3)$ & N3-Ru01-N2 & $81.0(3)$
\end{tabular}




\begin{tabular}{|c|c|c|c|}
\hline N4-Ru01-N6 & $91.7(3)$ & N5-Ru01-N6 & $176.2(3)$ \\
\hline N3-Ru01-N6 & $95.7(3)$ & N2-Ru01-N6 & $87.4(3)$ \\
\hline N4-Ru01-N1 & $97.8(3)$ & N5-Ru01-N1 & $97.8(3)$ \\
\hline N3-Ru01-N1 & $179.2(3)$ & N2-Ru01-N1 & $98.5(3)$ \\
\hline N6-Ru01-N1 & $84.9(3)$ & NOOJ-Ru02-N00A & $88.4(3)$ \\
\hline N00J-Ru02-N00X & $169.1(4)$ & N00A-Ru02-N00X & $91.1(4)$ \\
\hline N00J-Ru02-NO0S & $82.4(4)$ & N00A-Ru02-N00S & $81.6(4)$ \\
\hline N00X-Ru02-N00S & $86.7(5)$ & NOOJ-Ru02-NOOH & $97.5(3)$ \\
\hline NOOA-Ru02-NOOH & $97.6(3)$ & NOOX-Ru02-NOOH & $93.4(4)$ \\
\hline NOOS-Ru02-NOOH & 179.1(4) & N00J-Ru02-N00L & $90.5(3)$ \\
\hline NOOA-Ru02-N00L & $170.9(3)$ & N00X-Ru02-N00L & $88.3(4)$ \\
\hline NO0S-Ru02-N00L & $89.4(4)$ & N00H-Ru02-N00L & $91.5(3)$ \\
\hline F00E-P003-F00K & $89.5(6)$ & F00E-P003-F016 & $178.0(8)$ \\
\hline F00K-P003-F016 & $92.3(7)$ & F00E-P003-F00G & $89.6(5)$ \\
\hline F00K-P003-F00G & $89.3(6)$ & F016-P003-F00G & $89.5(6)$ \\
\hline F00E-P003-F00R & $90.7(7)$ & F00K-P003-F00R & $179.4(6)$ \\
\hline F016-P003-F00R & $87.6(8)$ & F00G-P003-F00R & $91.3(6)$ \\
\hline F00E-P003-F007 & $91.4(4)$ & F00K-P003-F007 & $91.3(6)$ \\
\hline F016-P003-F007 & $89.5(5)$ & F00G-P003-F007 & $178.8(5)$ \\
\hline F00R-P003-F007 & $88.1(5)$ & F01R-P004-F01B & $118.2(15)$ \\
\hline F01R-P004-F00M & $90.2(6)$ & F01B-P004-F00M & $90.1(6)$ \\
\hline F01R-P004-F2 & $162.9(15)$ & F01B-P004-F2 & $78.8(10)$ \\
\hline F00M-P004-F2 & $88.3(7)$ & F01R-P004-F00N & $90.2(7)$ \\
\hline F01B-P004-F00N & $89.9(6)$ & F00M-P004-F00N & $179.5(6)$ \\
\hline F2-P004-F00N & $91.2(7)$ & F01R-P004-F02P & $89.1(14)$ \\
\hline F01B-P004-F02P & $152.6(11)$ & F00M-P004-F02P & $90.7(7)$ \\
\hline F2-P004-F02P & $73.9(9)$ & F00N-P004-F02P & $89.1(7)$ \\
\hline F02I-P005-F015 & $93.6(9)$ & F02I-P005-F01G & $87.1(11)$ \\
\hline F015-P005-F01G & $87.2(7)$ & F02I-P005-F02N & $89.1(8)$ \\
\hline F015-P005-F02N & $175.1(11)$ & F01G-P005-F02N & $88.9(12)$ \\
\hline F02I-P005-F01E & $97.4(10)$ & F015-P005-F01E & $85.3(9)$ \\
\hline F01G-P005-F01E & $171.5(10)$ & F02N-P005-F01E & $98.4(13)$ \\
\hline F02I-P005-F008 & $174.0(8)$ & F015-P005-F008 & $90.1(7)$ \\
\hline F01G-P005-F008 & $88.3(6)$ & F02N-P005-F008 & $86.8(6)$ \\
\hline F01E-P005-F008 & $87.6(5)$ & F02C-P006-F021 & $87.0(6)$ \\
\hline F02C-P006-F6 & $172.9(16)$ & F021-P006-F6 & $93.2(12)$ \\
\hline F02C-P006-F01Z & $91.9(14)$ & F021-P006-F01Z & $103.5(13)$ \\
\hline F6-P006-F01Z & $81.2(13)$ & F02C-P006-F023 & $98.3(15)$ \\
\hline
\end{tabular}




\begin{tabular}{|c|c|c|c|}
\hline F021-P006-F023 & $91.0(12)$ & F6-P006-F023 & $88.7(10)$ \\
\hline F01Z-P006-F023 & $162.7(9)$ & F02C-P006-F00P & $83.1(6)$ \\
\hline F021-P006-F00P & $167.1(10)$ & F6-P006-F00P & $97.6(12)$ \\
\hline F01Z-P006-F00P & $85.1(6)$ & F023-P006-F00P & $82.3(6)$ \\
\hline C012-N3-C01C & $113.6(7)$ & C012-N3-C00U & $112.0(8)$ \\
\hline C01C-N3-C00U & $109.3(7)$ & C012-N3-Ru01 & $107.1(5)$ \\
\hline C01C-N3-Ru01 & $104.5(6)$ & C00U-N3-Ru01 & $110.0(5)$ \\
\hline C01K-N00A-C010 & $117.3(10)$ & C01K-N00A-Ru02 & $114.9(8)$ \\
\hline C010-N00A-Ru02 & $127.7(6)$ & C00Q-N4-C00O & $118.2(8)$ \\
\hline C00Q-N4-Ru01 & $112.6(6)$ & C00O-N4-Ru01 & $128.8(6)$ \\
\hline C00Z-N2-C014 & $118.3(8)$ & C00Z-N2-Ru01 & $130.7(7)$ \\
\hline C014-N2-Ru01 & $110.9(6)$ & C011-N5-C00Y & $117.9(8)$ \\
\hline C011-N5-Ru01 & $115.3(6)$ & C00Y-N5-Ru01 & $126.0(6)$ \\
\hline C01V-N6-C01Y & $106.0(9)$ & C01V-N6-Ru01 & $128.5(8)$ \\
\hline C01Y-N6-Ru01 & $125.2(7)$ & $\mathrm{C} 025-\mathrm{N} 00 \mathrm{H}-\mathrm{C} 01 \mathrm{~F}$ & $105.8(9)$ \\
\hline C025-NOOH-Ru02 & $127.1(7)$ & C01F-N00H-Ru02 & $127.1(6)$ \\
\hline C018-N1-C1O & $105.0(9)$ & C018-N1-Ru01 & $124.2(7)$ \\
\hline C1O-N1-Ru01 & $130.7(7)$ & C017-N00J-C019 & $117.0(9)$ \\
\hline C017-N00J-Ru02 & $129.4(7)$ & C019-N00J-Ru02 & $113.5(7)$ \\
\hline C02S-N00L-C2V & $76.4(19)$ & C02S-N00L-Ru02 & $138.8(10)$ \\
\hline C2V-N00L-Ru02 & $141.5(17)$ & N4-C00O-C00T & $122.1(9)$ \\
\hline N4-COOO-H0OO & 119.0 & COOT-C00O-H00O & 119.0 \\
\hline N4-C00Q-C013 & $122.0(9)$ & N4-C00Q-C012 & $116.5(8)$ \\
\hline C013-C00Q-C012 & $121.5(8)$ & C01L-N00S-C01S & $111.6(10)$ \\
\hline C01L-N00S-C02Q & $109.2(15)$ & C01S-N00S-C02Q & $115 .(2)$ \\
\hline C01L-N00S-Ru02 & $109.1(8)$ & C01S-N00S-Ru02 & $109.4(7)$ \\
\hline C02Q-N00S-Ru02 & $102.4(9)$ & C00O-C00T-C01J & $118.8(9)$ \\
\hline C00O-C0OT-H00T & 120.6 & C01J-C00T-H00T & 120.6 \\
\hline N3-C00U-C011 & $112.3(7)$ & N3-COOU-H0OA & 109.1 \\
\hline C011-C00U-H00A & 109.1 & N3-COOU-H00B & 109.1 \\
\hline C011-C00U-H00B & 109.1 & H0OA-COOU-H0OB & 107.9 \\
\hline C00Y-C00V-C01D & $118.6(10)$ & COOY-COOV-H0OV & 120.7 \\
\hline C01D-C00V-H00V & 120.7 & C018-N0W-C1W & $107.8(9)$ \\
\hline C018-N0W-H0W & 126.1 & C1W-N0W-H0W & 126.1 \\
\hline C028-N00X-C02O & $109.9(13)$ & C028-N00X-Ru02 & $138.3(10)$ \\
\hline C02O-N00X-Ru02 & $106.6(9)$ & N5-C00Y-C00V & $122.7(9)$ \\
\hline N5-C00Y-H00Y & 118.6 & COOV-COOY-H0OY & 118.6 \\
\hline N2-C00Z-C01H & $122.3(9)$ & N2-COOZ-H0OZ & 118.9 \\
\hline
\end{tabular}




\begin{tabular}{|c|c|c|c|}
\hline C01H-C00Z-H0OZ & 118.9 & N00A-C010-C01U & $122.3(10)$ \\
\hline N00A-C010-H010 & 118.8 & C01U-C010-H010 & 118.8 \\
\hline N5-C011-C01M & $121.4(9)$ & N5-C011-C00U & 116.2(9) \\
\hline C01M-C011-C00U & $122.3(9)$ & N3-C012-C00Q & $111.7(7)$ \\
\hline N3-C012-H01A & 109.3 & C00Q-C012-H01A & 109.3 \\
\hline N3-C012-H01B & 109.3 & C00Q-C012-H01B & 109.3 \\
\hline H01A-C012-H01B & 108.0 & C01V-N1A-C24 & $108.6(10)$ \\
\hline C01V-N1A-H01C & 125.7 & C24-N1A-H01C & 125.7 \\
\hline C01J-C013-C00Q & $119.0(10)$ & C01J-C013-H013 & 120.5 \\
\hline C00Q-C013-H013 & 120.5 & N2-C014-C01I & $120.9(9)$ \\
\hline N2-C014-C01C & $116.6(8)$ & C01I-C014-C01C & $122.4(9)$ \\
\hline N00J-C017-C01N & $123.7(11)$ & N00J-C017-H017 & 118.1 \\
\hline C01N-C017-H017 & 118.1 & NoW-C018-N1 & $110.7(10)$ \\
\hline N0W-C018-H018 & 124.6 & N1-C018-H018 & 124.6 \\
\hline N00J-C019-C01T & $122.4(12)$ & N00J-C019-C01L & $117.4(9)$ \\
\hline C01T-C019-C01L & $120.2(10)$ & N3-C01C-C014 & $108.8(7)$ \\
\hline N3-C01C-H01D & 109.9 & C014-C01C-H01D & 109.9 \\
\hline N3-C01C-H01E & 109.9 & C014-C01C-H01E & 109.9 \\
\hline H01D-C01C-H01E & 108.3 & C01M-C01D-C00V & $118.6(10)$ \\
\hline C01M-C01D-H01F & 120.7 & C00V-C01D-H01F & 120.7 \\
\hline C1X-C01F-N00H & 109.2(9) & C1X-C01F-H01G & 125.4 \\
\hline N00H-C01F-H01G & 125.4 & C01Q-C01H-C00Z & $119.5(9)$ \\
\hline C01Q-C01H-H01H & 120.2 & C00Z-C01H-H01H & 120.2 \\
\hline C01Q-C01I-C014 & $119.4(10)$ & C01Q-C01I-H01I & 120.3 \\
\hline C014-C01I-H01I & 120.3 & C013-C01J-C00T & 119.8(9) \\
\hline C013-C01J-H01J & 120.1 & C00T-C01J-H01J & 120.1 \\
\hline N00A-C01K-C3 & $121.1(13)$ & N00A-C01K-C01S & $117.2(13)$ \\
\hline C3-C01K-C01S & $121.7(13)$ & N00S-C01L-C019 & $112.7(8)$ \\
\hline N00S-C01L-H01K & 109.1 & C019-C01L-H01K & 109.1 \\
\hline N00S-C01L-H01L & 109.1 & C019-C01L-H01L & 109.1 \\
\hline H01K-C01L-H01L & 107.8 & C01D-C01M-C011 & $120.8(10)$ \\
\hline C01D-C01M-H01M & 119.6 & C011-C01M-H01M & 119.6 \\
\hline $\mathrm{C} 020-\mathrm{C} 01 \mathrm{~N}-\mathrm{C} 017$ & $119.2(12)$ & C020-C01N-H01N & 120.4 \\
\hline C017-C01N-H01N & 120.4 & C1W-C1O-N1 & $110.7(11)$ \\
\hline C1W-C1O-H1O & 124.6 & N1-C1O-H1O & 124.6 \\
\hline C025-N1P-C1X & $108.6(10)$ & C025-N1P-H1P & 125.7 \\
\hline C1X-N1P-H1P & 125.7 & C01H-C01Q-C01I & $119.6(10)$ \\
\hline C01H-C01Q-H01Q & 120.2 & C01I-C01Q-H01Q & 120.2 \\
\hline
\end{tabular}




\begin{tabular}{|c|c|c|c|}
\hline N00S-C01S-C01K & $112.9(9)$ & N00S-C01S-H01R & 109.0 \\
\hline C01K-C01S-H01R & 109.0 & N00S-C01S-H01S & 109.0 \\
\hline C01K-C01S-H01S & 109.0 & H01R-C01S-H01S & 107.8 \\
\hline C019-C01T-C020 & $117.6(11)$ & C019-C01T-H01T & 121.2 \\
\hline C020-C01T-H01T & 121.2 & C02J-C01U-C010 & $119.6(14)$ \\
\hline C02J-C01U-H01U & 120.2 & C010-C01U-H01U & 120.2 \\
\hline N1A-C01V-N6 & $110.5(11)$ & N1A-C01V-H01V & 124.7 \\
\hline N6-C01V-H01V & 124.7 & C1O-C1W-N0W & $105.8(10)$ \\
\hline C1O-C1W-H1W & 127.1 & NOW-C1W-H1W & 127.1 \\
\hline N1P-C1X-C01F & $105.3(10)$ & N1P-C1X-H1X & 127.4 \\
\hline C01F-C1X-H1X & 127.4 & N6-C01Y-C24 & $109.8(11)$ \\
\hline N6-C01Y-H01Y & 125.1 & C24-C01Y-H01Y & 125.1 \\
\hline C01N-C020-C01T & $120.0(11)$ & $\mathrm{C} 01 \mathrm{~N}-\mathrm{C} 020-\mathrm{H} 020$ & 120.0 \\
\hline C01T-C020-H020 & 120.0 & C01Y-C24-N1A & 105.1(12) \\
\hline $\mathrm{C} 01 \mathrm{Y}-\mathrm{C} 24-\mathrm{H} 24$ & 127.5 & N1A-C24-H24 & 127.5 \\
\hline $\mathrm{N} 00 \mathrm{H}-\mathrm{C} 025-\mathrm{N} 1 \mathrm{P}$ & $111.2(11)$ & N00H-C025-H025 & 124.4 \\
\hline N1P-C025-H025 & 124.4 & C02H-C028-N00X & $120.2(18)$ \\
\hline $\mathrm{C} 02 \mathrm{H}-\mathrm{C} 028-\mathrm{H} 028$ & 119.9 & N00X-C028-H028 & 119.9 \\
\hline $\mathrm{C} 028-\mathrm{C} 02 \mathrm{H}-\mathrm{C} 02 \mathrm{R}$ & $104.5(18)$ & $\mathrm{C} 028-\mathrm{C} 02 \mathrm{H}-\mathrm{H} 02 \mathrm{H}$ & 127.8 \\
\hline $\mathrm{C} 02 \mathrm{R}-\mathrm{C} 02 \mathrm{H}-\mathrm{H} 02 \mathrm{H}$ & 127.8 & C3-C02J-C01U & 119.1(13) \\
\hline $\mathrm{C} 3-\mathrm{C} 02 \mathrm{~J}-\mathrm{H} 02 \mathrm{~J}$ & 120.5 & C01U-C02J-H02J & 120.5 \\
\hline C02S-N2M-C2V & $72.0(18)$ & $\mathrm{N} 00 \mathrm{X}-\mathrm{C} 02 \mathrm{O}-\mathrm{C} 02 \mathrm{Q}$ & $107.4(15)$ \\
\hline N00X-C02O-C02R & $99.5(15)$ & C02Q-C02O-C02R & $146.7(15)$ \\
\hline N00S-C02Q-C02O & $97.3(17)$ & $\mathrm{C} 02 \mathrm{H}-\mathrm{C} 02 \mathrm{R}-\mathrm{C} 02 \mathrm{O}$ & $88.2(11)$ \\
\hline N00L-C02S-N2M & $123.2(17)$ & NOOL-C02S-C2V & $66.9(19)$ \\
\hline $\mathrm{N} 2 \mathrm{M}-\mathrm{C} 02 \mathrm{~S}-\mathrm{C} 2 \mathrm{~V}$ & $62 .(2)$ & N2M-C2V-N00L & $80.3(11)$ \\
\hline $\mathrm{N} 2 \mathrm{M}-\mathrm{C} 2 \mathrm{~V}-\mathrm{C} 02 \mathrm{~S}$ & $46.1(10)$ & N00L-C2V-C02S & $36.7(8)$ \\
\hline $\mathrm{C} 02 \mathrm{~J}-\mathrm{C} 3-\mathrm{C} 01 \mathrm{~K}$ & $120.5(12)$ & $\mathrm{C} 02 \mathrm{~J}-\mathrm{C} 3-\mathrm{H} 3$ & 119.8 \\
\hline C01K-C3-H3 & 119.8 & & \\
\hline
\end{tabular}

Table S13. Anisotropic atomic displacement parameters $\left(\AA^{2}\right)$ for [Ru(TPA)(imidazole $\left.)_{2}\right]\left(\mathrm{PF}_{6}\right)_{2}$.

The anisotropic atomic displacement factor exponent takes the form: $-2 \pi^{2}\left[\mathrm{~h}^{2} \mathrm{a}^{* 2} \mathrm{U}_{11}+\ldots+2\right.$ h k a $\left.{ }^{*} b^{*} U_{12}\right]$

\begin{tabular}{lllllll}
\hline & $\mathrm{U}_{11}$ & $\mathrm{U}_{22}$ & $\mathrm{U}_{33}$ & $\mathrm{U}_{23}$ & $\mathrm{U}_{13}$ & $\mathrm{U}_{12}$ \\
$\mathrm{Ru} 01$ & $0.0391(4)$ & $0.0300(4)$ & $0.0339(4)$ & $-0.0101(3)$ & $0.0204(3)$ & $-0.0102(3)$ \\
$\mathrm{Ru} 02$ & $0.0306(4)$ & $0.0491(5)$ & $0.0343(4)$ & $0.0150(3)$ & $0.0076(3)$ & $0.0111(3)$
\end{tabular}




\begin{tabular}{|c|c|c|c|c|c|c|}
\hline & $\mathrm{U}_{11}$ & $\mathrm{U}_{22}$ & $\mathrm{U}_{33}$ & $\mathrm{U}_{23}$ & $\mathrm{U}_{13}$ & $\mathrm{U}_{12}$ \\
\hline P003 & $0.0381(13)$ & $0.081(2)$ & $0.0397(14)$ & $0.0239(13)$ & $-0.0020(11)$ & $-0.0268(13)$ \\
\hline P004 & $0.099(2)$ & $0.0467(15)$ & $0.0496(16)$ & $-0.0164(13)$ & $0.0387(17)$ & $-0.0106(15)$ \\
\hline P005 & $0.0504(17)$ & $0.085(2)$ & $0.065(2)$ & $0.0201(17)$ & $0.0238(15)$ & $-0.0019(15)$ \\
\hline P006 & $0.239(7)$ & $0.0497(19)$ & $0.055(2)$ & $0.0059(16)$ & $0.063(3)$ & $-0.021(3)$ \\
\hline F007 & $0.076(5)$ & $0.125(7)$ & $0.044(4)$ & $0.001(4)$ & $0.017(4)$ & $-0.042(5)$ \\
\hline F008 & $0.090(5)$ & $0.046(3)$ & $0.068(4)$ & $0.002(3)$ & $0.031(4)$ & $-0.008(3)$ \\
\hline N3 & $0.043(4)$ & $0.032(4)$ & $0.053(5)$ & $-0.013(3)$ & $0.030(4)$ & $-0.012(3)$ \\
\hline NOOA & $0.047(4)$ & $0.034(4)$ & $0.028(4)$ & $0.002(3)$ & $0.011(3)$ & $-0.004(3)$ \\
\hline N4 & $0.041(4)$ & $0.036(4)$ & $0.036(4)$ & $-0.014(3)$ & $0.019(3)$ & $-0.010(3)$ \\
\hline $\mathrm{N} 2$ & $0.045(4)$ & $0.030(4)$ & $0.039(4)$ & $-0.009(3)$ & $0.019(3)$ & $-0.005(3)$ \\
\hline N5 & $0.043(4)$ & $0.028(3)$ & $0.032(4)$ & $-0.009(3)$ & $0.020(3)$ & $-0.006(3)$ \\
\hline F00E & $0.131(7)$ & $0.109(6)$ & $0.051(4)$ & $-0.010(4)$ & $0.040(5)$ & $-0.075(6)$ \\
\hline N6 & $0.049(5)$ & $0.048(5)$ & $0.037(4)$ & $-0.011(3)$ & $0.020(4)$ & $-0.014(4)$ \\
\hline F00G & $0.061(4)$ & $0.186(10)$ & $0.067(5)$ & $0.056(6)$ & $0.019(4)$ & $-0.036(5)$ \\
\hline $\mathrm{NOOH}$ & $0.047(4)$ & $0.032(4)$ & $0.039(4)$ & $0.004(3)$ & $0.019(4)$ & $0.005(3)$ \\
\hline N1 & $0.046(4)$ & $0.039(4)$ & $0.047(4)$ & $-0.001(3)$ & $0.023(4)$ & $-0.009(3)$ \\
\hline NOOJ & $0.044(4)$ & $0.031(4)$ & $0.049(5)$ & $0.013(3)$ & $0.021(4)$ & $0.016(3)$ \\
\hline F00K & $0.131(8)$ & $0.156(10)$ & $0.046(4)$ & $0.021(5)$ & $0.007(5)$ & $0.054(7)$ \\
\hline N00L & $0.042(4)$ & $0.068(6)$ & $0.032(4)$ & $0.000(4)$ & $0.012(3)$ & $0.018(4)$ \\
\hline F00M & $0.184(10)$ & $0.053(4)$ & $0.083(5)$ & $-0.009(4)$ & $0.077(6)$ & $-0.012(5)$ \\
\hline F00N & $0.189(11)$ & $0.068(5)$ & $0.096(6)$ & $-0.021(5)$ & $0.090(7)$ & $-0.028(6)$ \\
\hline $\mathrm{COOO}$ & $0.036(4)$ & $0.044(5)$ & $0.035(5)$ & $-0.012(4)$ & $0.016(4)$ & $-0.006(4)$ \\
\hline F00P & $0.158(9)$ & $0.067(5)$ & $0.096(6)$ & $-0.002(5)$ & $0.057(6)$ & $0.018(6)$ \\
\hline COOQ & $0.050(5)$ & $0.040(5)$ & $0.037(5)$ & $-0.013(4)$ & $0.022(4)$ & $-0.008(4)$ \\
\hline F00R & $0.105(7)$ & $0.198(12)$ & $0.093(7)$ & $0.094(8)$ & $0.041(6)$ & $0.057(8)$ \\
\hline NO0S & $0.028(4)$ & $0.124(10)$ & $0.038(5)$ & $0.033(5)$ & $0.002(4)$ & $0.003(5)$ \\
\hline COOT & $0.038(5)$ & $0.053(6)$ & $0.046(6)$ & $-0.013(4)$ & $0.010(4)$ & $0.001(4)$ \\
\hline $\mathrm{COOU}$ & $0.066(7)$ & $0.047(6)$ & $0.058(6)$ & $-0.003(5)$ & $0.044(6)$ & $-0.011(5)$ \\
\hline $\mathrm{COOV}$ & $0.054(6)$ & $0.038(5)$ & $0.051(6)$ & $-0.009(4)$ & $0.013(5)$ & $0.002(4)$ \\
\hline NOW & $0.068(6)$ & $0.062(6)$ & $0.077(7)$ & $0.022(5)$ & $0.044(6)$ & $-0.002(5)$ \\
\hline N00X & $0.043(5)$ & $0.076(7)$ & $0.145(11)$ & $0.072(8)$ & $0.048(6)$ & $0.031(5)$ \\
\hline $\mathrm{COOY}$ & $0.050(5)$ & $0.038(5)$ & $0.040(5)$ & $-0.013(4)$ & $0.019(4)$ & $-0.007(4)$ \\
\hline $\mathrm{COOZ}$ & $0.051(5)$ & $0.034(5)$ & $0.039(5)$ & $-0.010(4)$ & $0.017(4)$ & $-0.002(4)$ \\
\hline $\mathrm{C} 010$ & $0.047(5)$ & $0.034(5)$ & $0.046(5)$ & $0.003(4)$ & $0.017(4)$ & $0.002(4)$ \\
\hline $\mathrm{C} 011$ & $0.053(5)$ & $0.032(4)$ & $0.048(5)$ & $-0.012(4)$ & $0.032(5)$ & $-0.003(4)$ \\
\hline C012 & $0.056(6)$ & $0.032(5)$ & $0.065(6)$ & $-0.019(4)$ & $0.037(5)$ & $-0.014(4)$ \\
\hline N1A & $0.080(7)$ & $0.099(9)$ & $0.037(5)$ & $-0.029(5)$ & $0.016(5)$ & $-0.006(6)$ \\
\hline
\end{tabular}




\begin{tabular}{|c|c|c|c|c|c|c|}
\hline & $\mathrm{U}_{11}$ & $\mathrm{U}_{22}$ & $\mathrm{U}_{33}$ & $\mathrm{U}_{23}$ & $\mathrm{U}_{13}$ & $\mathrm{U}_{12}$ \\
\hline $\mathrm{C} 013$ & $0.055(6)$ & $0.038(5)$ & $0.053(6)$ & $-0.013(4)$ & $0.026(5)$ & $-0.005(4)$ \\
\hline $\mathrm{C} 014$ & $0.047(5)$ & $0.046(5)$ & $0.053(6)$ & $-0.013(4)$ & $0.029(5)$ & $-0.011(4)$ \\
\hline F015 & $0.089(7)$ & $0.094(7)$ & $0.321(19)$ & $0.038(9)$ & $0.118(10)$ & $0.030(6)$ \\
\hline F016 & $0.209(12)$ & $0.177(11)$ & $0.049(5)$ & $0.036(5)$ & $-0.026(6)$ & $-0.157(10)$ \\
\hline $\mathrm{C} 017$ & $0.044(5)$ & $0.029(4)$ & $0.068(7)$ & $0.015(4)$ & $0.023(5)$ & $0.014(4)$ \\
\hline $\mathrm{C} 018$ & $0.052(6)$ & $0.058(6)$ & $0.050(6)$ & $0.012(5)$ & $0.031(5)$ & $0.000(5)$ \\
\hline $\mathrm{C} 019$ & $0.080(7)$ & $0.034(5)$ & $0.052(6)$ & $0.016(4)$ & $0.038(6)$ & $0.026(5)$ \\
\hline F01B & $0.283(18)$ & $0.141(10)$ & $0.068(6)$ & $-0.040(6)$ & $0.039(8)$ & $-0.144(11)$ \\
\hline $\mathrm{C} 01 \mathrm{C}$ & $0.038(5)$ & $0.044(5)$ & $0.062(6)$ & $-0.015(5)$ & $0.027(5)$ & $-0.013(4)$ \\
\hline C01D & $0.078(8)$ & $0.053(6)$ & $0.042(6)$ & $0.001(5)$ & $0.021(6)$ & $0.014(6)$ \\
\hline F01E & $0.35(2)$ & $0.229(14)$ & $0.167(11)$ & $-0.160(11)$ & $0.202(14)$ & $-0.251(16)$ \\
\hline $\mathrm{C} 01 \mathrm{~F}$ & $0.041(5)$ & $0.045(5)$ & $0.040(5)$ & $0.002(4)$ & $0.013(4)$ & $0.002(4)$ \\
\hline $\mathrm{F} 01 \mathrm{G}$ & $0.299(17)$ & $0.039(4)$ & $0.297(18)$ & $0.053(7)$ & $0.245(16)$ & $0.034(7)$ \\
\hline $\mathrm{C} 01 \mathrm{H}$ & $0.058(6)$ & $0.041(5)$ & $0.047(6)$ & $-0.011(4)$ & $0.024(5)$ & $0.005(4)$ \\
\hline C01I & $0.052(6)$ & $0.051(6)$ & $0.074(7)$ & $-0.018(5)$ & $0.038(6)$ & $-0.009(5)$ \\
\hline $\mathrm{C} 01 \mathrm{~J}$ & $0.054(6)$ & $0.043(5)$ & $0.054(6)$ & $-0.006(5)$ & $0.013(5)$ & $0.011(5)$ \\
\hline $\mathrm{C} 01 \mathrm{~K}$ & $0.107(10)$ & $0.044(6)$ & $0.031(5)$ & $-0.001(4)$ & $0.009(6)$ & $-0.024(6)$ \\
\hline $\mathrm{C} 01 \mathrm{~L}$ & $0.082(8)$ & $0.051(6)$ & $0.031(5)$ & $0.007(4)$ & $0.016(5)$ & $0.026(6)$ \\
\hline $\mathrm{C} 01 \mathrm{M}$ & $0.068(7)$ & $0.041(5)$ & $0.045(6)$ & $0.006(4)$ & $0.026(5)$ & $0.005(5)$ \\
\hline $\mathrm{C} 01 \mathrm{~N}$ & $0.062(7)$ & $0.027(5)$ & $0.098(9)$ & $0.012(5)$ & $0.040(7)$ & $0.008(4)$ \\
\hline $\mathrm{C} 1 \mathrm{O}$ & $0.046(5)$ & $0.034(5)$ & $0.072(7)$ & $-0.003(5)$ & $0.025(5)$ & $-0.013(4)$ \\
\hline N1P & $0.131(11)$ & $0.072(7)$ & $0.045(6)$ & $-0.024(5)$ & $0.040(6)$ & $-0.037(7)$ \\
\hline C01Q & $0.057(7)$ & $0.059(7)$ & $0.071(7)$ & $-0.012(6)$ & $0.034(6)$ & $0.007(5)$ \\
\hline F01R & $0.43(3)$ & $0.149(12)$ & $0.64(4)$ & $0.172(18)$ & $0.50(3)$ & $0.155(15)$ \\
\hline $\mathrm{C} 01 \mathrm{~S}$ & $0.134(14)$ & $0.059(8)$ & $0.050(7)$ & $0.017(6)$ & $-0.037(8)$ & $-0.044(9)$ \\
\hline $\mathrm{C} 01 \mathrm{~T}$ & $0.101(10)$ & $0.035(5)$ & $0.067(7)$ & $0.015(5)$ & $0.050(7)$ & $0.021(6)$ \\
\hline $\mathrm{C} 01 \mathrm{U}$ & $0.110(10)$ & $0.047(6)$ & $0.072(8)$ & $0.006(6)$ & $0.061(8)$ & $0.018(6)$ \\
\hline $\mathrm{C} 01 \mathrm{~V}$ & $0.051(6)$ & $0.065(7)$ & $0.044(6)$ & $-0.025(5)$ & $0.017(5)$ & $-0.007(5)$ \\
\hline $\mathrm{C} 1 \mathrm{~W}$ & $0.058(7)$ & $0.042(6)$ & $0.096(10)$ & $0.013(6)$ & $0.033(7)$ & $-0.010(5)$ \\
\hline C1X & $0.055(6)$ & $0.071(8)$ & $0.038(5)$ & $0.008(5)$ & $0.012(5)$ & $-0.012(5)$ \\
\hline $\mathrm{C} 01 \mathrm{Y}$ & $0.095(9)$ & $0.050(6)$ & $0.037(6)$ & $-0.006(5)$ & $0.011(6)$ & $-0.011(6)$ \\
\hline F01Z & $0.43(3)$ & $0.32(2)$ & $0.136(11)$ & $0.143(14)$ & $0.189(16)$ & $0.31(2)$ \\
\hline $\mathrm{C} 020$ & $0.124(12)$ & $0.029(5)$ & $0.105(11)$ & $0.022(6)$ & $0.084(10)$ & $0.017(6)$ \\
\hline F021 & $0.55(3)$ & $0.276(18)$ & $0.31(2)$ & $-0.248(17)$ & $0.40(2)$ & $-0.31(2)$ \\
\hline F023 & $0.163(12)$ & $0.242(17)$ & $0.081(7)$ & $0.007(9)$ & $0.051(8)$ & $0.049(12)$ \\
\hline $\mathrm{C} 24$ & $0.108(11)$ & $0.069(8)$ & $0.044(7)$ & $-0.017(6)$ & $0.004(7)$ & $-0.006(8)$ \\
\hline $\mathrm{C} 025$ & $0.143(13)$ & $0.040(6)$ & $0.048(6)$ & $-0.006(5)$ & $0.051(8)$ & $0.001(7)$ \\
\hline
\end{tabular}




$\begin{array}{lllllll} & \mathrm{U}_{11} & \mathrm{U}_{22} & \mathrm{U}_{33} & \mathrm{U}_{23} & \mathrm{U}_{13} & \mathrm{U}_{12} \\ \mathrm{C} 028 & 0.094(11) & 0.072(9) & 0.071(9) & 0.025(7) & -0.002(8) & -0.007(8) \\ \mathrm{F} 02 \mathrm{C} & 0.76(4) & 0.070(7) & 0.56(3) & -0.136(13) & 0.63(4) & -0.158(15) \\ \mathrm{C} 02 \mathrm{H} & 0.110(14) & 0.38(4) & 0.084(12) & 0.102(19) & 0.018(11) & -0.12(2) \\ \mathrm{F} 02 \mathrm{I} & 0.210(16) & 0.39(3) & 0.091(8) & 0.096(12) & 0.093(10) & 0.150(17) \\ \mathrm{C} 02 \mathrm{~J} & 0.26(3) & 0.049(7) & 0.079(10) & 0.013(7) & 0.110(15) & 0.038(11) \\ \mathrm{N} 2 \mathrm{M} & 0.25(3) & 0.088(11) & 0.37(4) & 0.084(17) & 0.25(3) & 0.026(14) \\ \mathrm{F} 02 \mathrm{~N} & 0.076(7) & 0.69(5) & 0.072(7) & 0.045(14) & 0.024(6) & 0.129(15) \\ \mathrm{C} 02 \mathrm{O} & 0.081(11) & 0.128(15) & 0.089(11) & 0.015(11) & 0.027(9) & 0.022(10) \\ \mathrm{F} 02 \mathrm{P} & 0.106(9) & 0.175(13) & 0.173(13) & 0.033(11) & 0.024(9) & -0.040(9) \\ \mathrm{C} 02 \mathrm{Q} & 0.037(8) & 0.57(6) & 0.081(12) & 0.15(2) & 0.020(8) & 0.027(17) \\ \mathrm{C} 02 \mathrm{R} & 0.028(8) & 0.37(5) & 0.46(6) & -0.31(5) & 0.012(17) & -0.034(16) \\ \mathrm{C} 02 \mathrm{~S} & 0.072(11) & 0.027(7) & 0.62(6) & -0.017(16) & 0.16(2) & 0.006(7) \\ \mathrm{C} 2 \mathrm{~V} & 0.21(3) & 0.68(8) & 0.050(11) & -0.03(2) & 0.048(15) & -0.28(4) \\ \mathrm{F} 2 & 0.102(9) & 0.148(12) & 0.30(2) & 0.026(14) & 0.083(12) & 0.006(8) \\ \mathrm{F} 6 & 0.217(18) & 0.190(16) & 0.205(17) & 0.038(13) & -0.060(14) & -0.127(14)\end{array}$

Table S14. Hydrogen atomic coordinates and isotropic atomic displacement parameters $\left(\AA^{2}\right)$ for $\left.[\mathrm{Ru}(\mathrm{TPA}) \text { (imidazole })_{2}\right]\left(\mathrm{PF}_{6}\right)_{2}$.

\begin{tabular}{lllllll}
\hline $\mathrm{C} 3$ & $0.22(2)$ & $0.054(8)$ & $0.040(7)$ & $-0.013(6)$ & $0.056(10)$ & $-0.021(10)$
\end{tabular}

$\begin{array}{lllll} & \mathrm{x} / \mathrm{a} & \mathrm{y} / \mathrm{b} & \mathrm{z} / \mathrm{c} & \mathrm{U}(\mathrm{eq}) \\ \text { H00O } & 0.0646 & 0.1824 & 0.3240 & 0.045 \\ \text { H00T } & 0.0046 & 0.2762 & 0.3335 & 0.058 \\ \text { H00A } & 0.3038 & 0.1939 & 0.1831 & 0.061 \\ \text { H00B } & 0.2684 & 0.2622 & 0.1855 & 0.061 \\ \text { H00V } & -0.0214 & 0.1248 & 0.0528 & 0.061 \\ \text { H0W } & 0.0868 & 0.0094 & 0.4313 & 0.078 \\ \text { H00Y } & 0.0587 & 0.1009 & 0.1801 & 0.051 \\ \text { H00Z } & 0.2646 & 0.0008 & 0.2717 & 0.05 \\ \text { H010 } & 0.9246 & 0.2963 & 0.6911 & 0.052 \\ \text { H01A } & 0.2908 & 0.3067 & 0.2900 & 0.057 \\ \text { H01B } & 0.3245 & 0.2734 & 0.3724 & 0.057 \\ \text { H01C } & 0.3680 & 0.1815 & 0.5931 & 0.09 \\ \text { H013 } & 0.1887 & 0.3694 & 0.3138 & 0.057 \\ \text { H017 } & 0.9306 & 0.4550 & 0.6824 & 0.057\end{array}$




$\begin{array}{lllll} & \mathrm{x} / \mathrm{a} & \mathrm{y} / \mathrm{b} & \mathrm{z} / \mathrm{c} & \mathrm{U}(\mathrm{eq}) \\ \mathrm{H} 018 & 0.1607 & 0.1024 & 0.4334 & 0.06 \\ \mathrm{H} 01 \mathrm{D} & 0.4029 & 0.1932 & 0.3754 & 0.055 \\ \mathrm{H} 01 \mathrm{E} & 0.4131 & 0.2116 & 0.2988 & 0.055 \\ \mathrm{H} 01 \mathrm{~F} & 0.0285 & 0.1953 & -0.0120 & 0.071 \\ \text { H01G } & 0.8573 & 0.2886 & 0.7740 & 0.052 \\ \text { H01H } & 0.3705 & -0.0485 & 0.2561 & 0.057 \\ \text { H01I } & 0.4899 & 0.1149 & 0.2966 & 0.066 \\ \text { H01J } & 0.0677 & 0.3709 & 0.3290 & 0.065 \\ \text { H01K } & 0.6698 & 0.4623 & 0.4588 & 0.069 \\ \text { H01L } & 0.7074 & 0.4175 & 0.4153 & 0.069 \\ \text { H01M } & 0.1543 & 0.2397 & 0.0545 & 0.06 \\ \text { H01N } & 0.9932 & 0.5234 & 0.6321 & 0.072 \\ \text { H1O } & 0.1268 & 0.0060 & 0.2465 & 0.061 \\ \text { H1P } & 0.9275 & 0.4422 & 0.8937 & 0.098 \\ \text { H01Q } & 0.4848 & 0.0079 & 0.2712 & 0.072 \\ \text { H01R } & 0.7081 & 0.3236 & 0.4206 & 0.128 \\ \text { H01S } & 0.6665 & 0.2851 & 0.4661 & 0.128 \\ \text { H01T } & 0.8029 & 0.5058 & 0.4258 & 0.075 \\ \text { H01U } & 0.9818 & 0.2166 & 0.6491 & 0.082 \\ \text { H01V } & 0.2833 & 0.2227 & 0.4703 & 0.065 \\ \text { H1W } & 0.0671 & -0.0548 & 0.3180 & 0.078 \\ \text { H1X } & 0.9366 & 0.3285 & 0.9062 & 0.069 \\ \text { H01Y } & 0.3528 & 0.0524 & 0.4424 & 0.08 \\ \text { H020 } & 0.9297 & 0.5521 & 0.5059 & 0.089 \\ \text { H24 } & 0.4119 & 0.0740 & 0.5826 & 0.099 \\ \text { H025 } & 0.8534 & 0.4727 & 0.7636 & 0.088 \\ \text { H028 } & 0.7356 & 0.2462 & 0.7013 & 0.11 \\ \text { H02H } & 0.6125 & 0.2364 & 0.7207 & 0.24 \\ \text { H02J } & 0.9152 & 0.1791 & 0.5243 & 0.139 \\ \text { H3 } & 0.7996 & 0.2264 & 0.4431 & 0.123\end{array}$




\section{Part D. Spectral Data for 1, 2 and 3}

Ao-02-95-pure
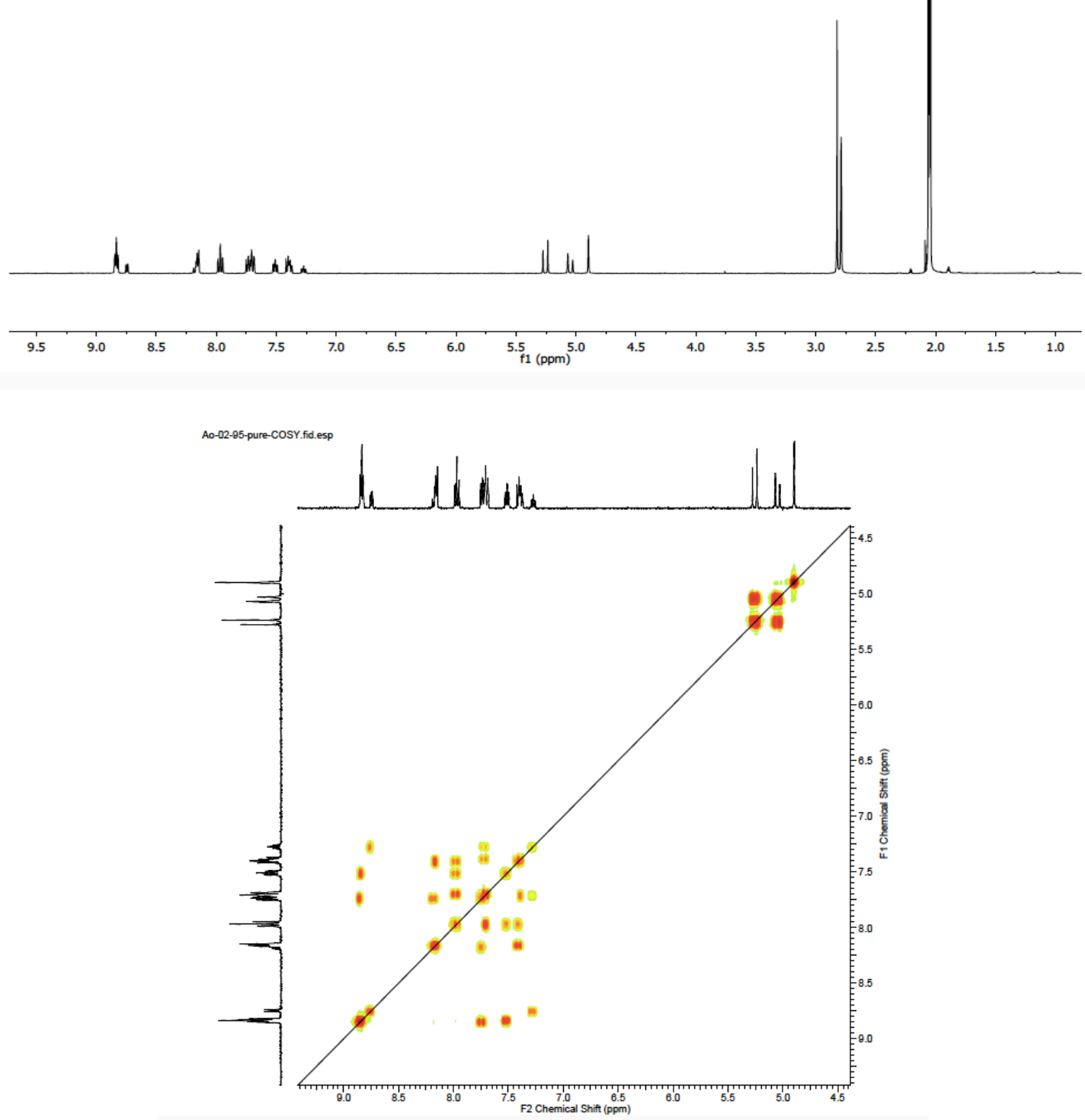

Figure S1. 1H NMR of 1 (top) COSY (bottom) in $\left(\mathrm{CD}_{3}\right)_{2} \mathrm{CO}$ 


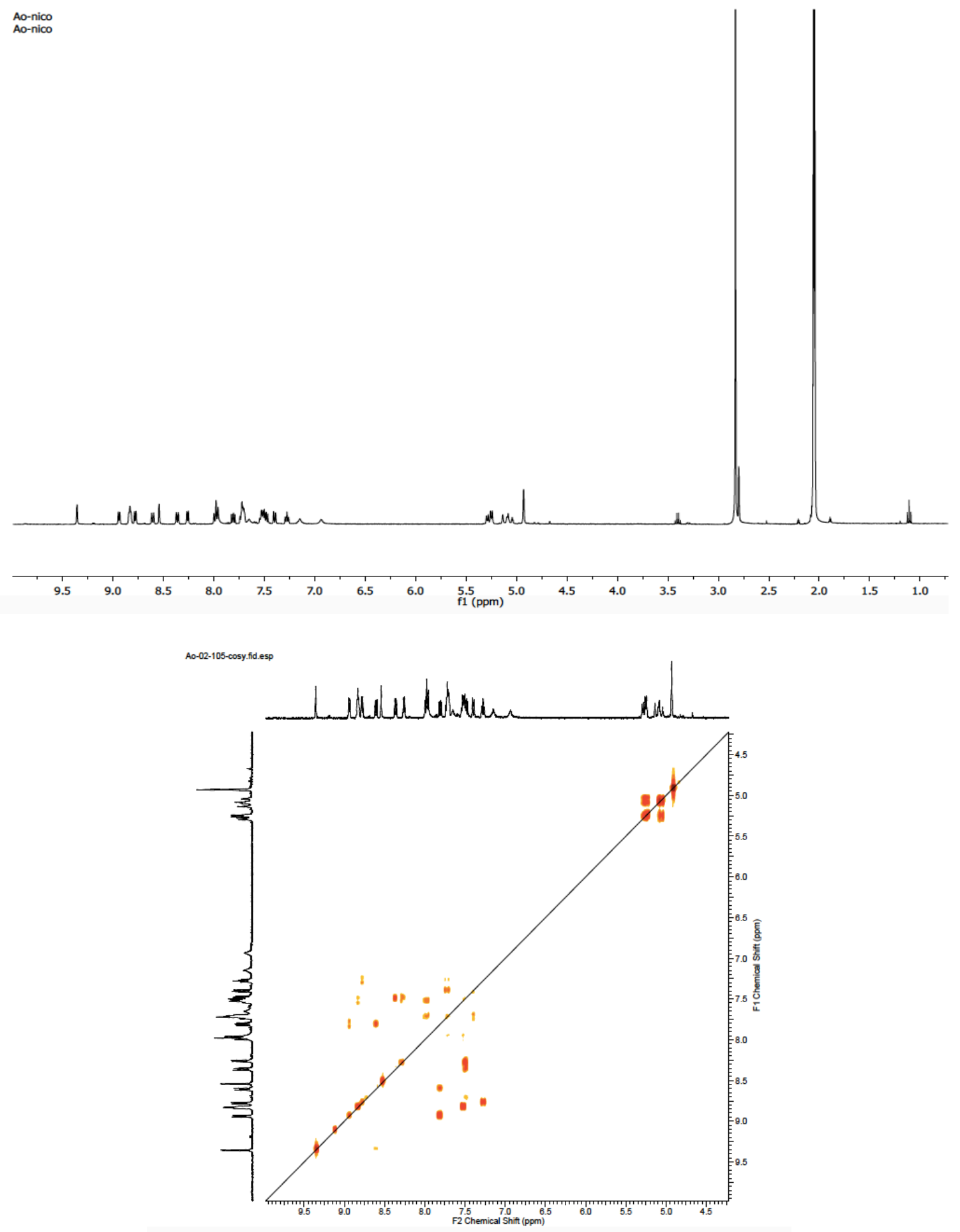

Figure S2. 1H NMR of 2 (top) COSY (bottom) in $\left(\mathrm{CD}_{3}\right)_{2} \mathrm{CO}$ 


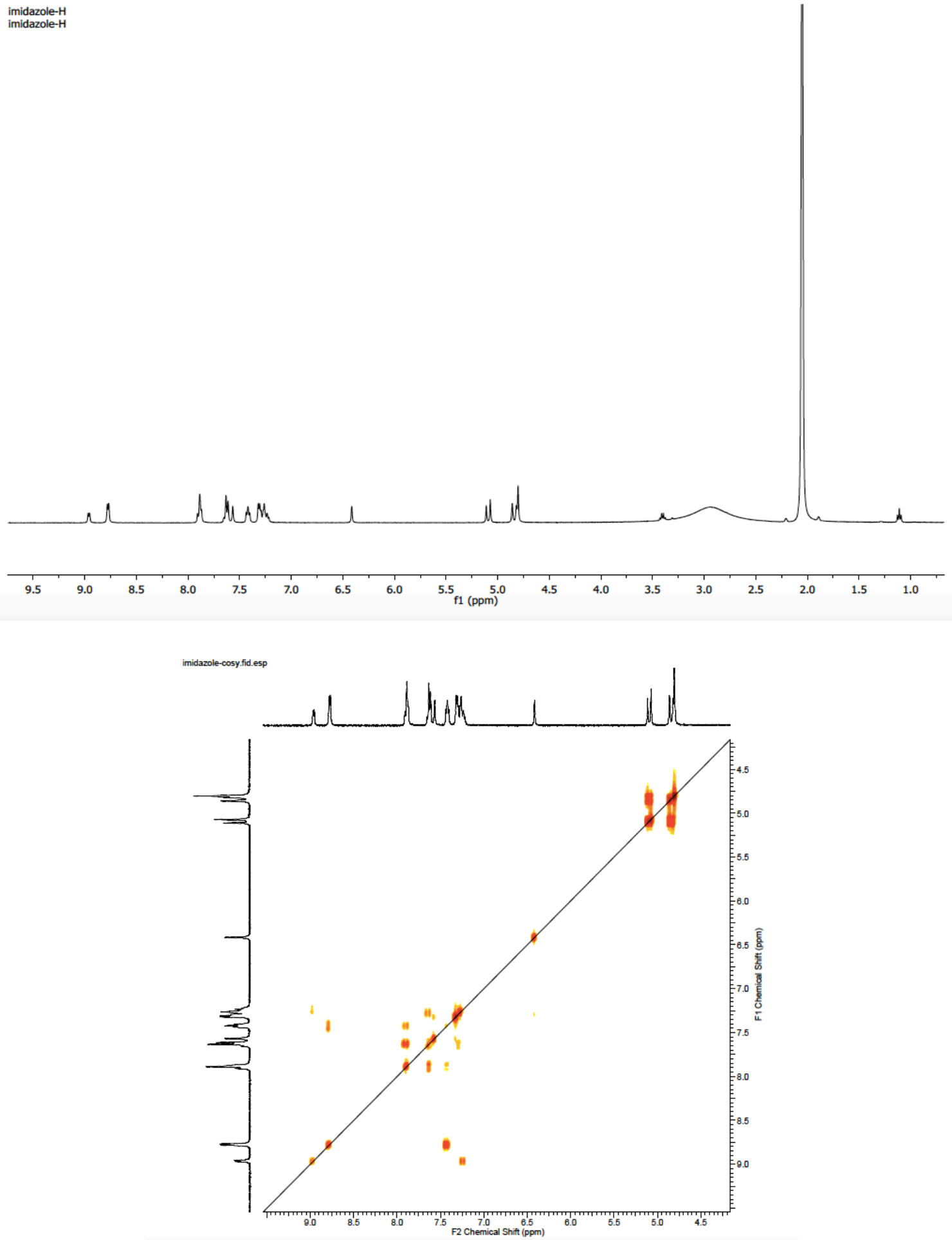

Figure S3. 1H NMR of 3 (top) COSY (bottom) in $\left(\mathrm{CD}_{3}\right)_{2} \mathrm{CO}$ 
Figure S4. NOESY data for 1 irradiating at 8.73 (A), 7.50 (B) and 7.27(C) ppm.

A

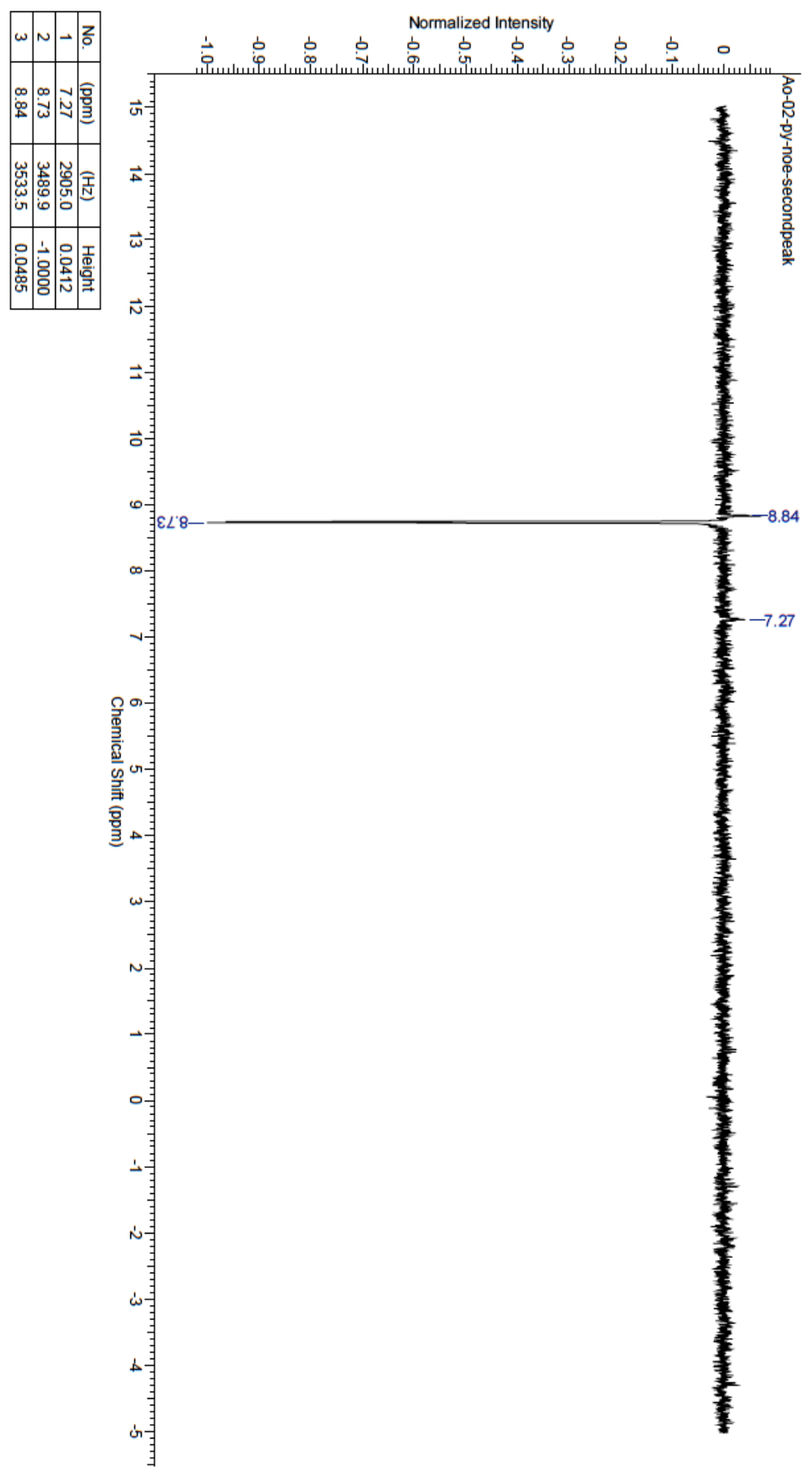


B

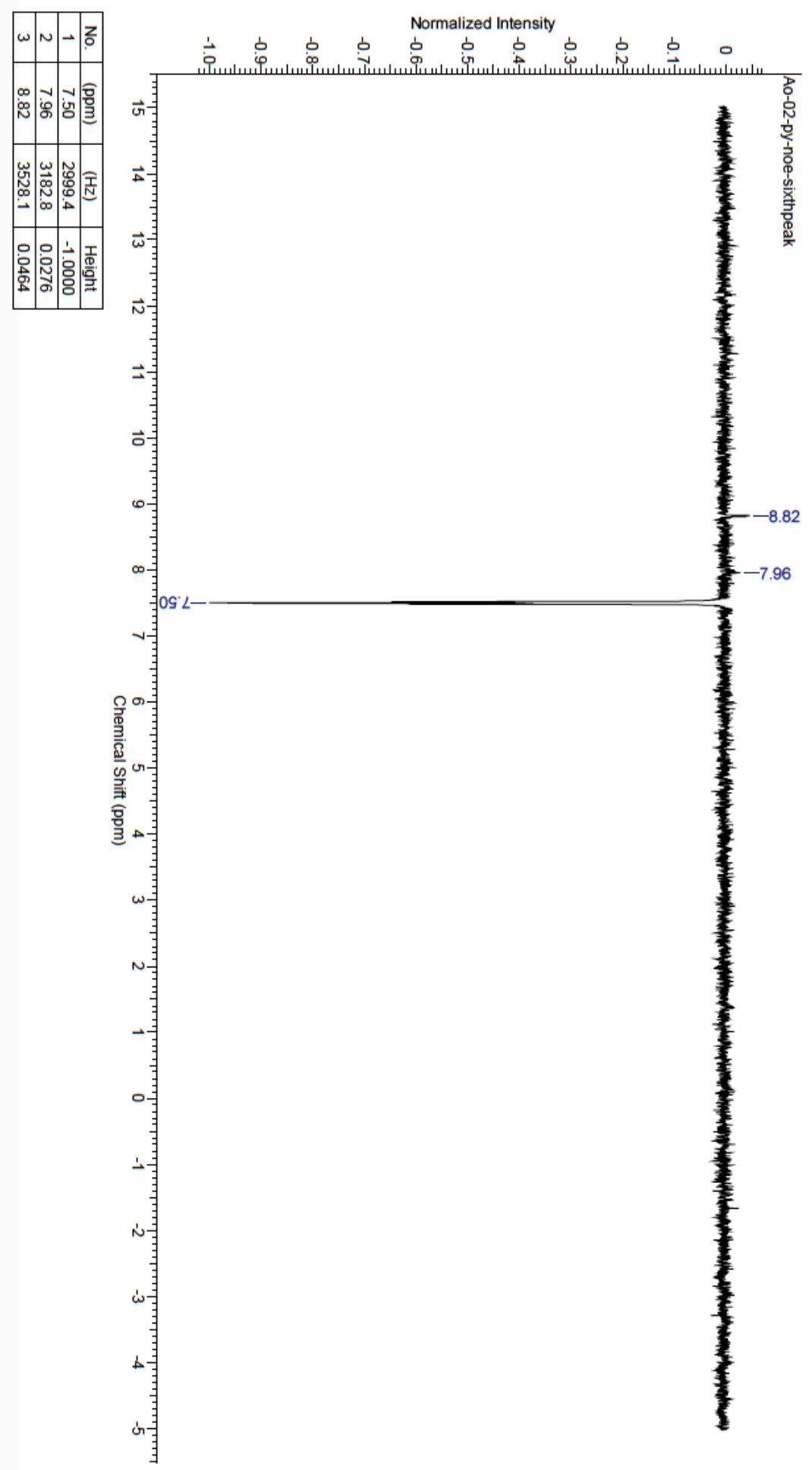

34 


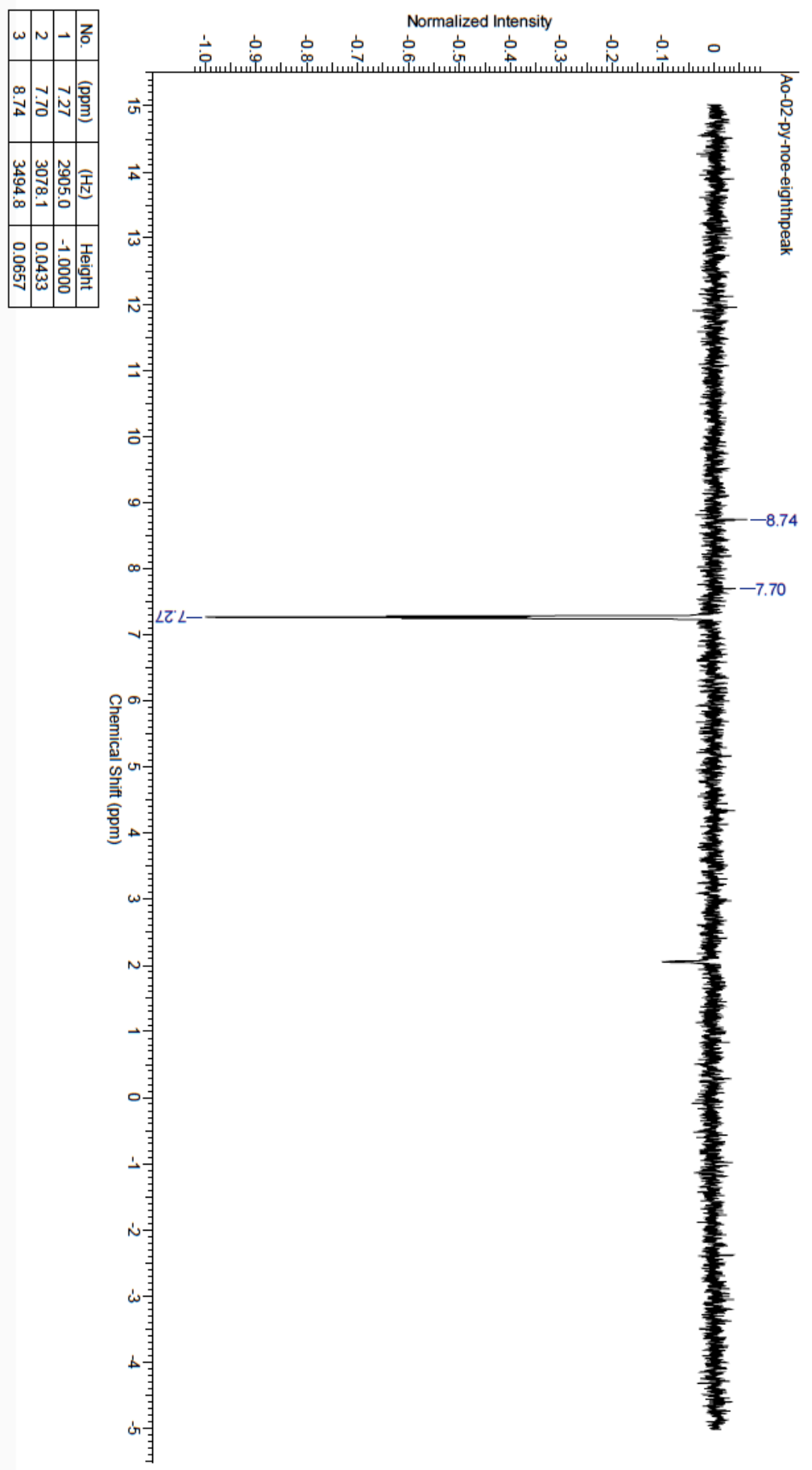



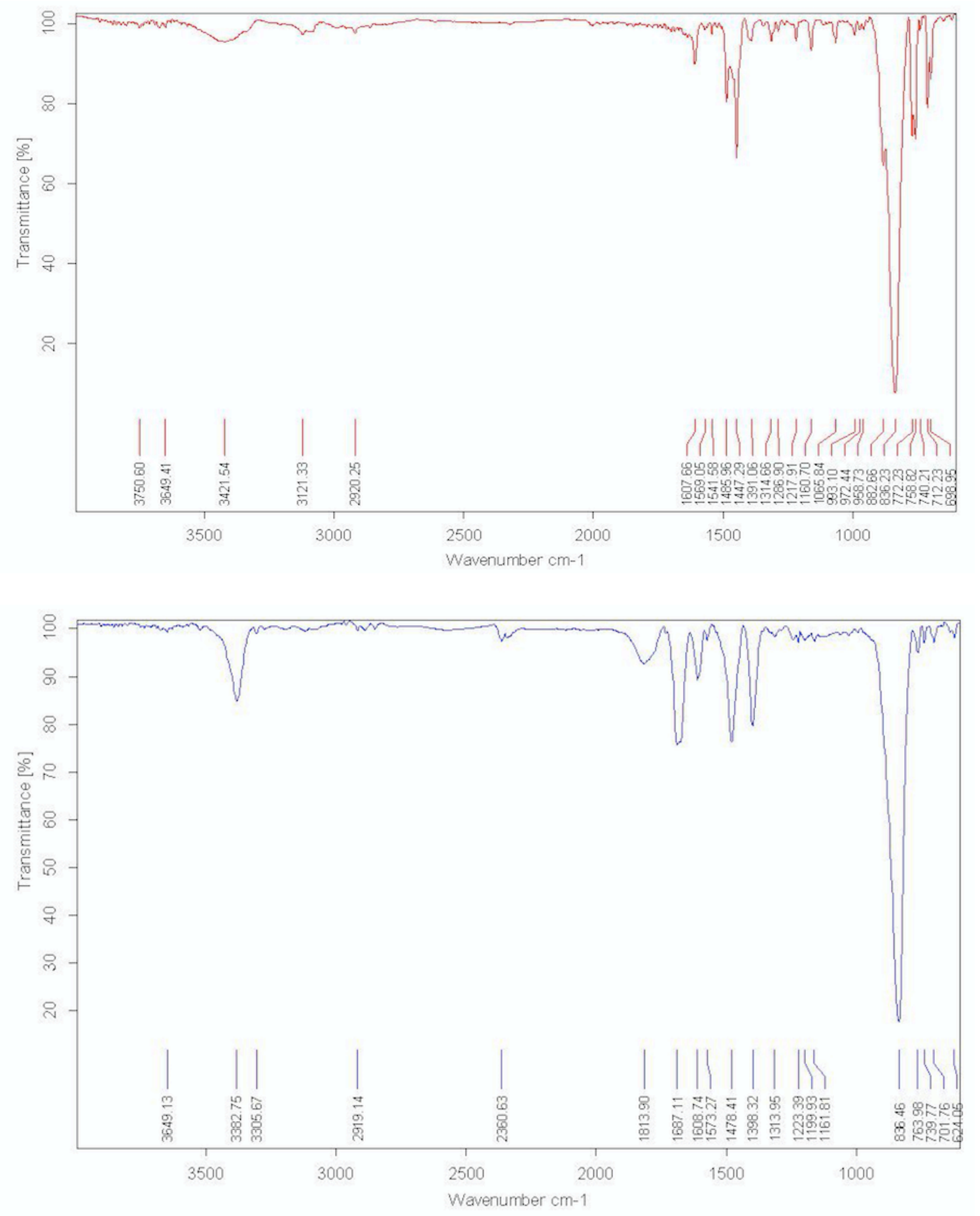

Figure S5. IR (KBr) of 1 (top) and 2 (bottom) 


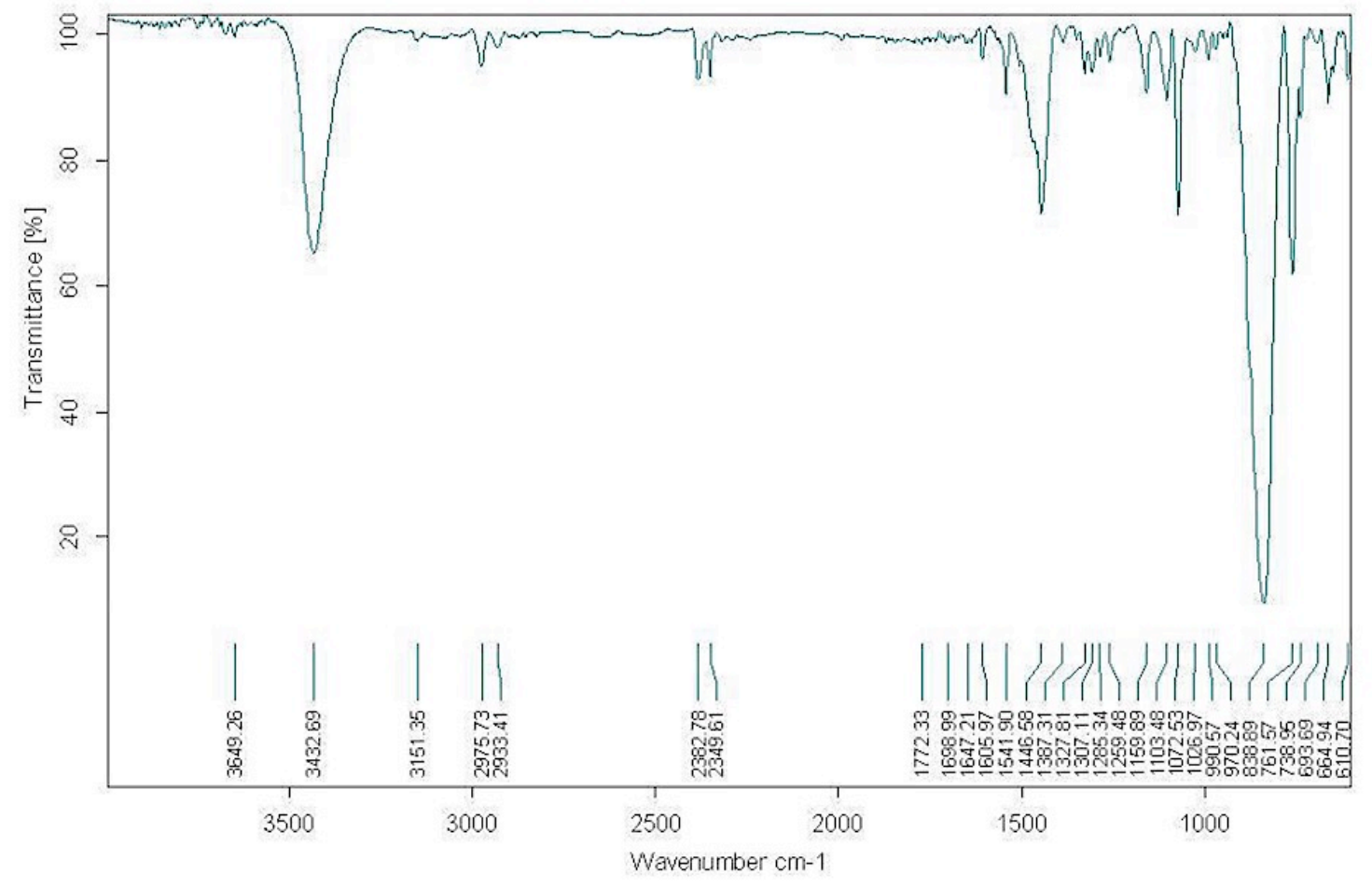

Figure S6. IR (KBr) of 3 


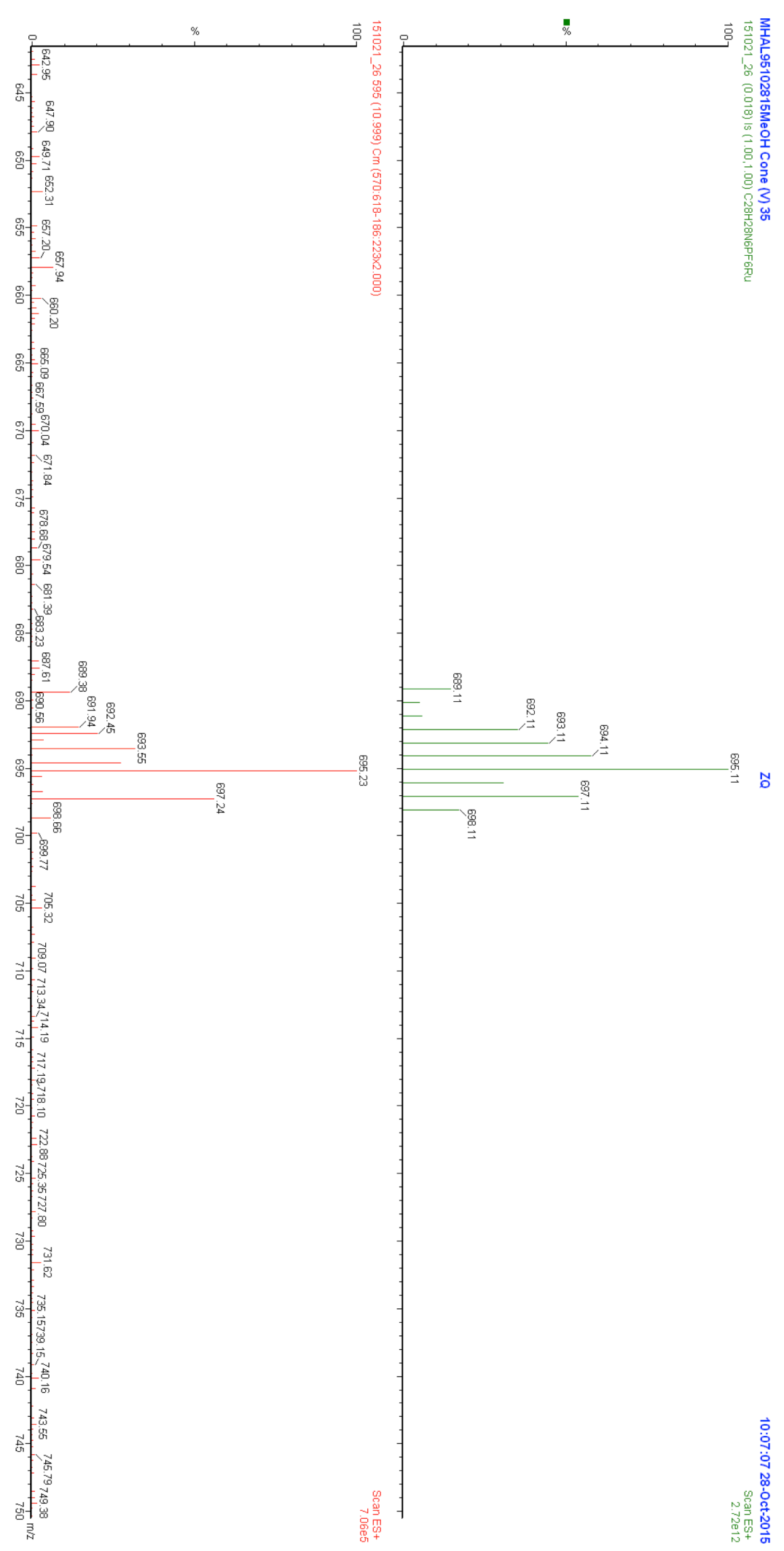

Figure S7. Mass spectrum calculated (right) and observed (left) of the cation $\left\{\left[\mathrm{Ru}(\mathrm{TPA})(\mathrm{py})_{2}\right]\left(\mathrm{PF}_{6}\right)\right\}^{+}$ 


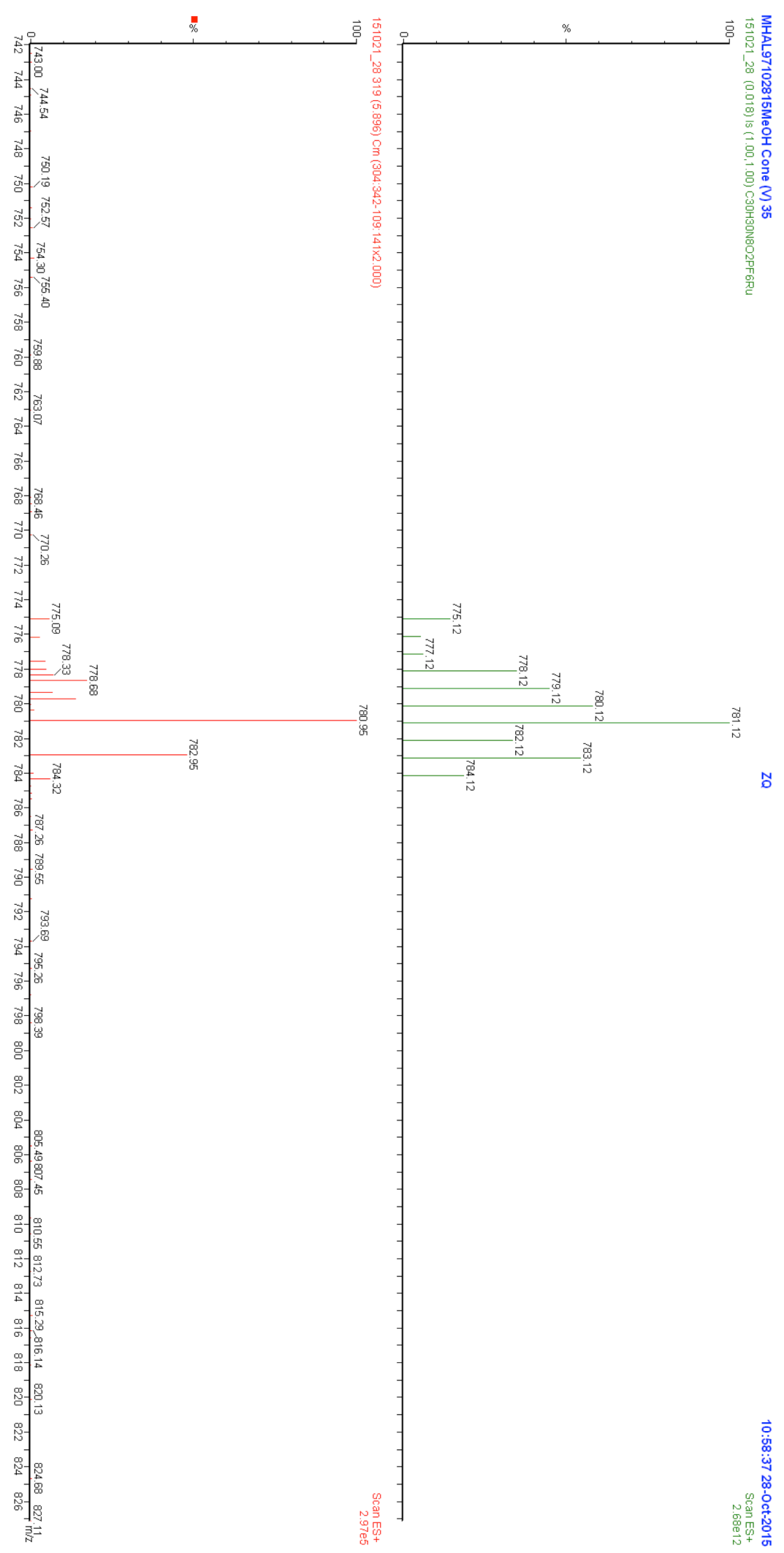

Figure S8. Mass spectrum calculated (right) and observed (left) of the cation $\left\{\left[\mathrm{Ru}(\mathrm{TPA})(\mathrm{NA})_{2}\right]\left(\mathrm{PF}_{6}\right)\right\}^{+}$ 


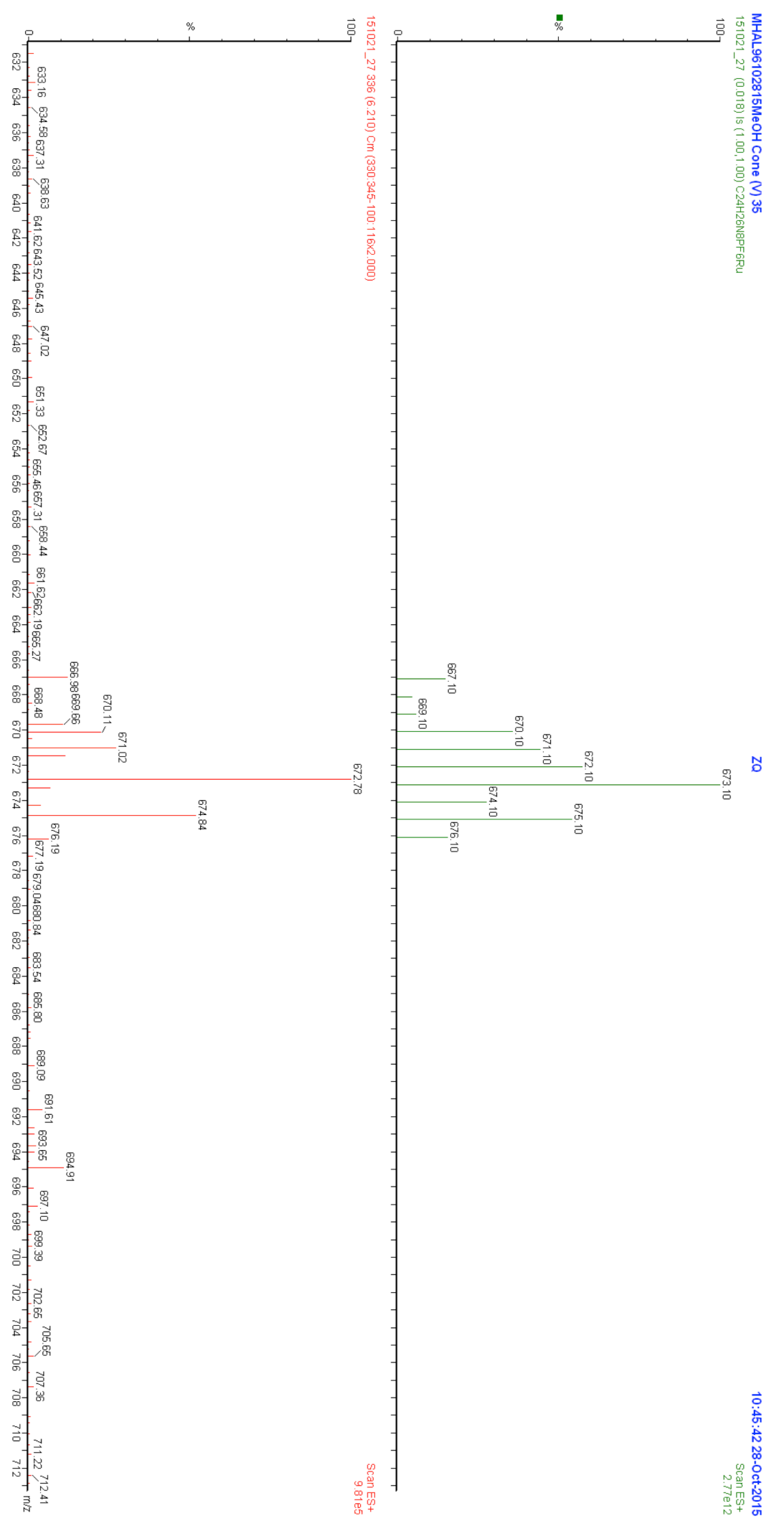

Figure S9. Mass spectrum calculated (right) and observed (left) of the cation $\left\{\left[\mathrm{Ru}(\mathrm{TPA})(\mathrm{Im})_{2}\right]\left(\mathrm{PF}_{6}\right)\right\}^{+}$ 

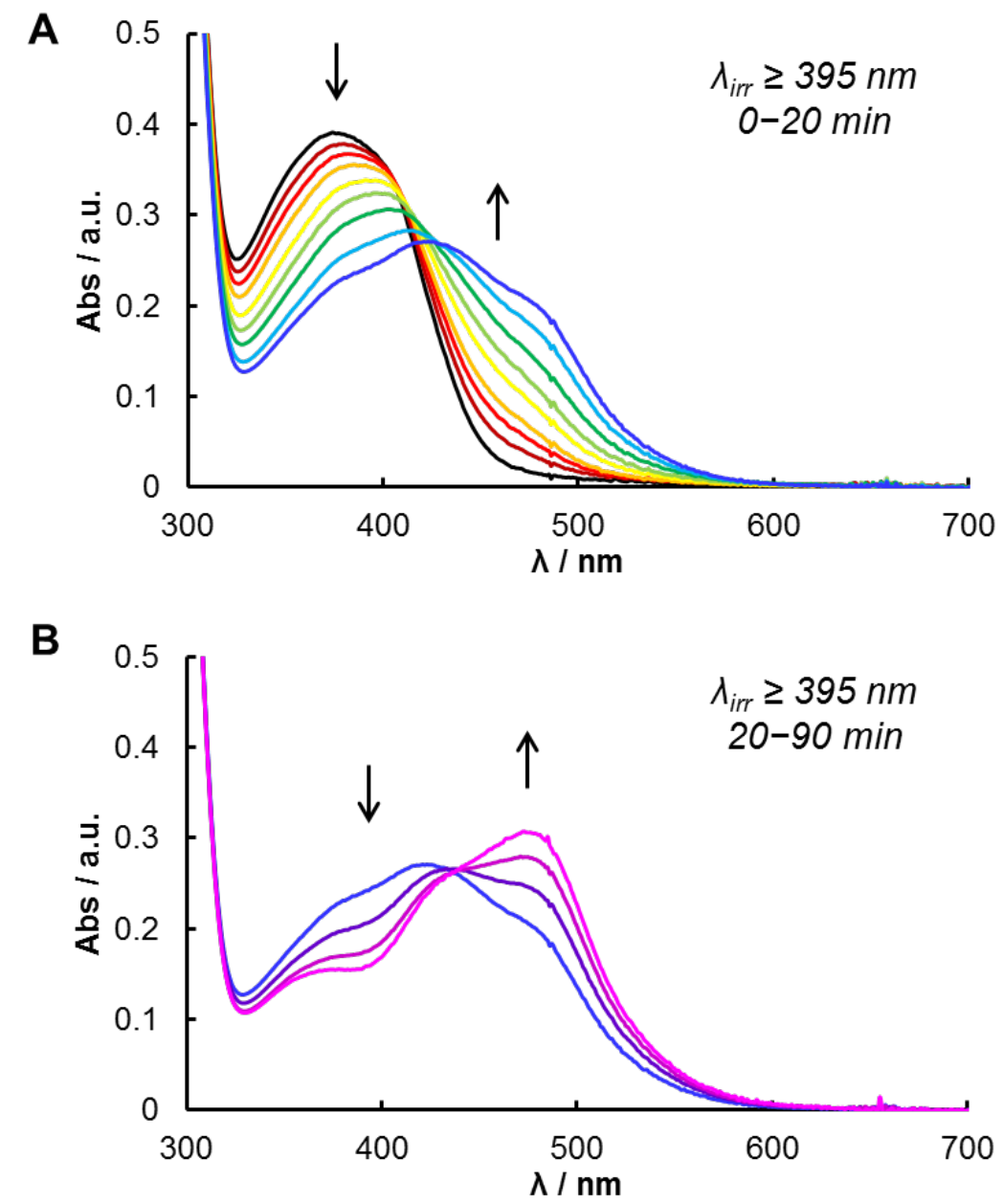

Figure S10. Electronic absorption spectra of 2 in $\mathrm{CH}_{2} \mathrm{Cl}_{2}$ with $10 \mathrm{mM} \mathrm{Bu} 4 \mathrm{NCl}$ irradiated with $\lambda \geq 395 \mathrm{~nm}$ for $0-20 \mathrm{~min}$ (A) and 20-90 $\min (\mathrm{B})$. 

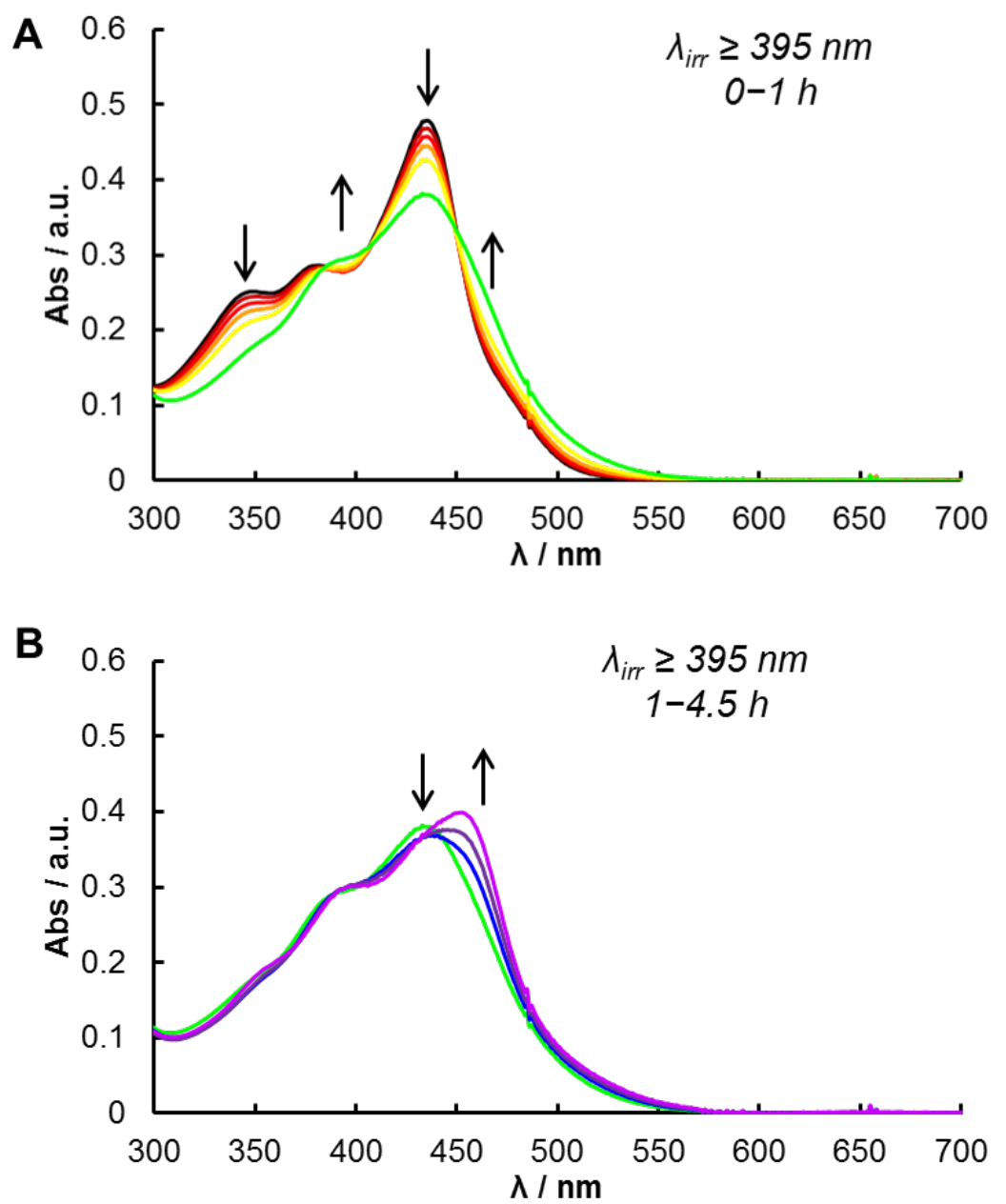

Figure S11. Electronic absorption spectra of 3 in $\mathrm{CH}_{2} \mathrm{Cl}_{2}$ with $10 \mathrm{mM} \mathrm{Bu} \mathrm{mCl}_{4}$ irradiated with $\lambda \geq 395 \mathrm{~nm}$ for $0-1 \mathrm{~h}(\mathrm{~A})$ and $1-4.5 \mathrm{~h}(\mathrm{~B})$. 
Figure S12. (A) ${ }^{1} \mathrm{H}$ NMR spectra of complex 1 in $\mathrm{D}_{2} \mathrm{O}\left(<10 \%\right.$ acetone- $\left.\mathrm{d}_{6}\right)$ upon visible light irradiation $\left(\lambda_{\text {irr }} \geq 395 \mathrm{~nm}\right)$ at $\mathrm{t}=0,60,180,240 \mathrm{~min}$ and overnight; the labeled A represents a new signal from the new $\mathrm{D}_{2} \mathrm{O}$-substituted complex; the labeled $\mathrm{Y}$ represents the peaks from free pyridine that release during irradiation (B) Schematic representation of ligand exchange of complex 1

A.

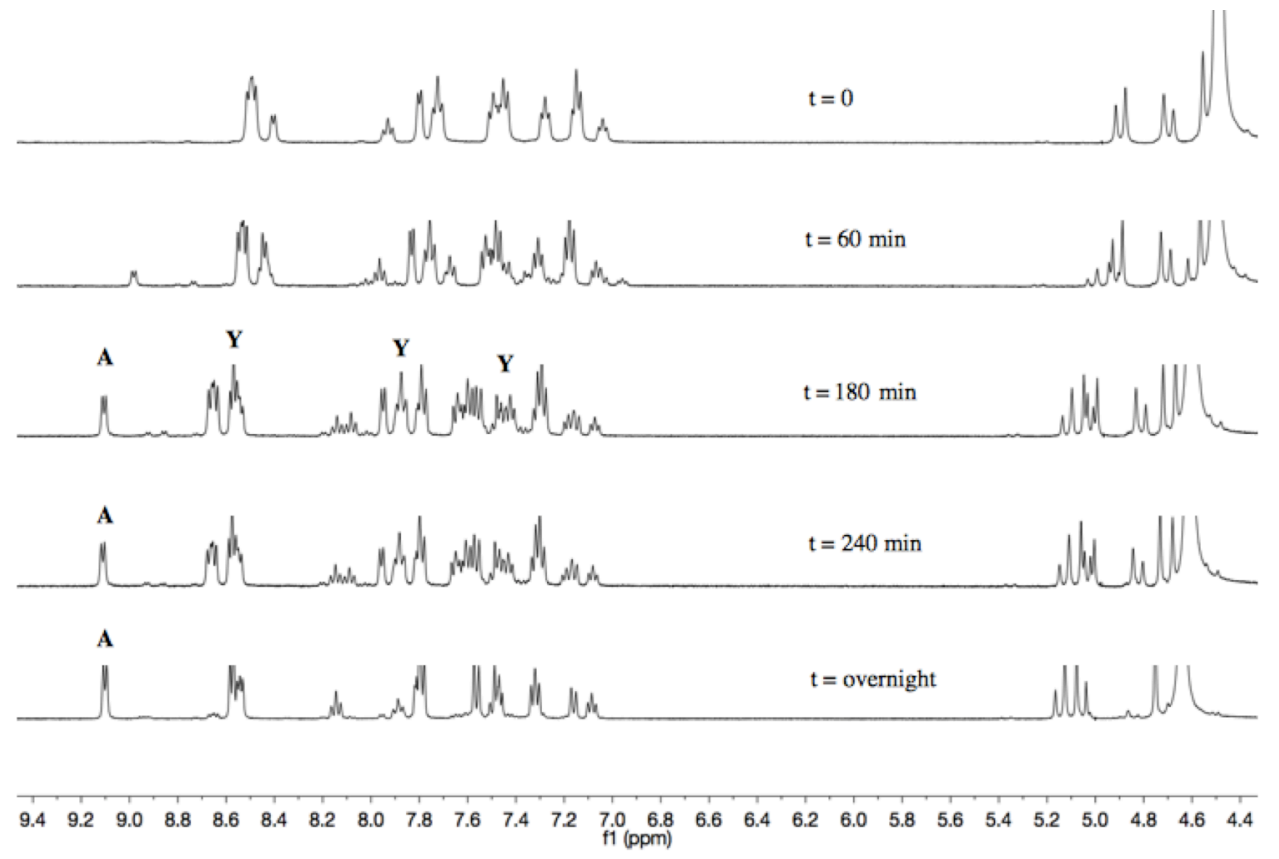

B.

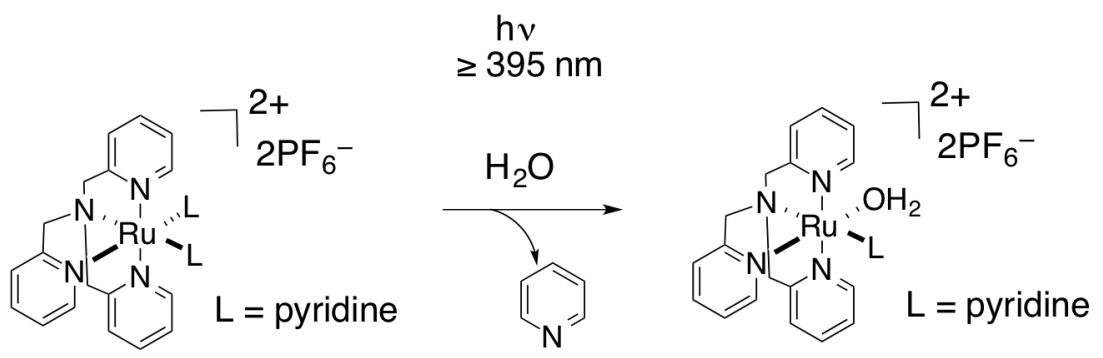


Figure S13. ${ }^{1} \mathrm{H}$ NMR spectra of complex 2 in $\mathrm{D}_{2} \mathrm{O}\left(<10 \%\right.$ acetone- $\left.\mathrm{d}_{6}\right)$ upon visible light irradiation $\left(\lambda_{\text {irr }} \geq 395 \mathrm{~nm}\right)$ at $\mathrm{t}=0,120,180 \mathrm{~min}$ and overnight; the labeled $\mathrm{M}$ represents new signals from the new $\mathrm{D}_{2} \mathrm{O}$-substituted complex; the labeled Q represents the peaks from starting material (B) Schematic representation of ligand exchange of complex 2

A.

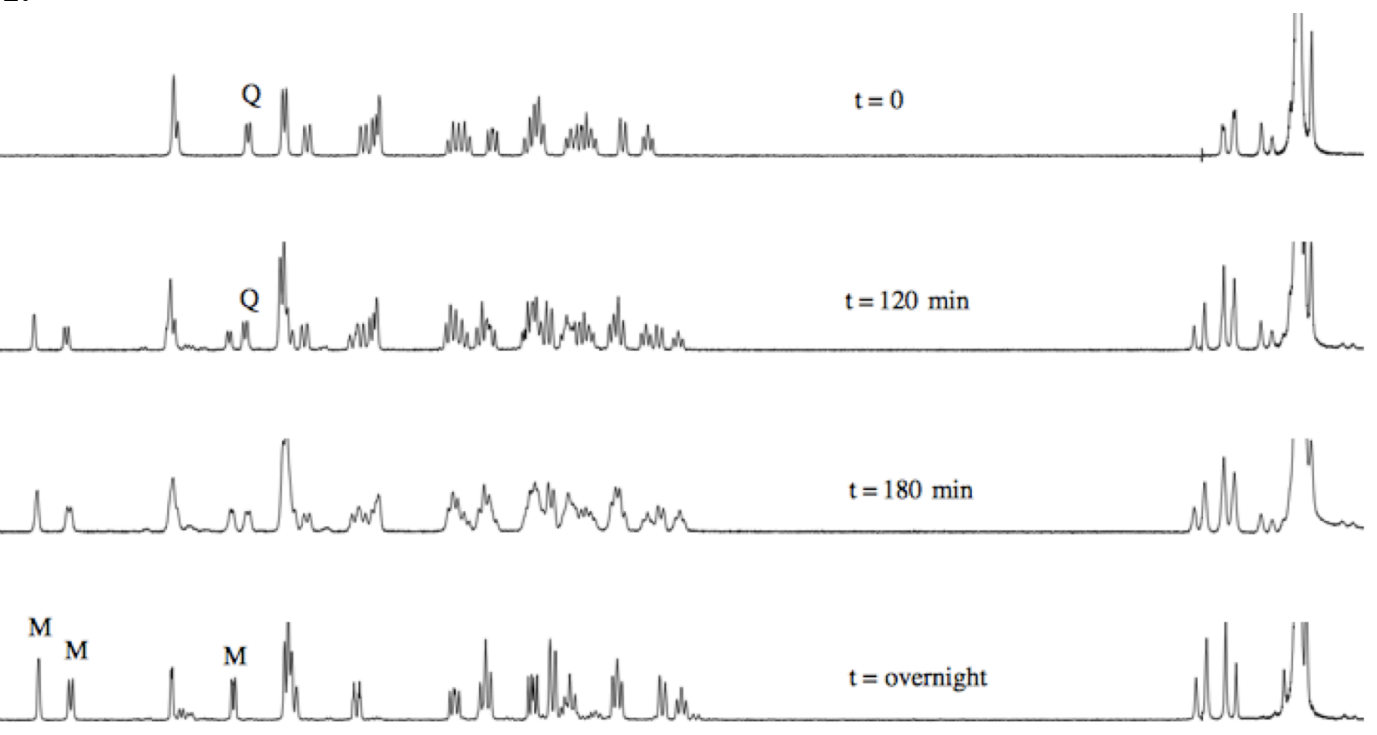

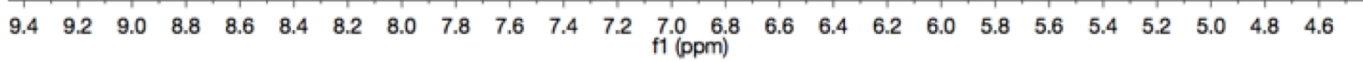

B.

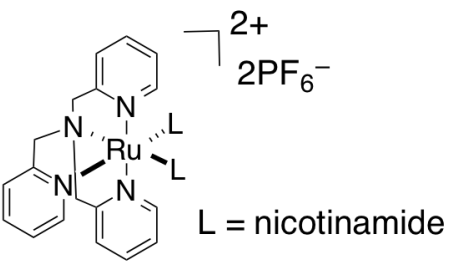<smiles></smiles>

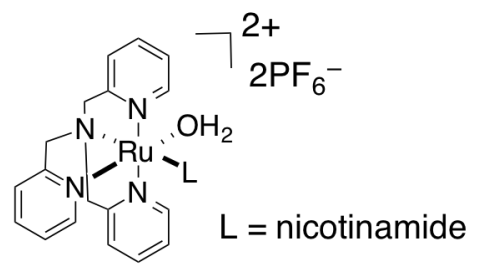


Figure S14. (A) ${ }^{1} \mathrm{H}$ NMR spectra of complex 3 in $\mathrm{D}_{2} \mathrm{O}(<10 \%$ acetone) upon visible light irradiation $\left(\lambda_{\text {irr }} \geq 395 \mathrm{~nm}\right)$ at $\mathrm{t}=0,60,120,180,240 \mathrm{~min}$ and overnight; the labeled $\mathrm{X}$ represents the signal for the 2-position $\mathrm{C}-\mathrm{H}$ on the imidazole; the labeled $\mathrm{Y}$ represents a new signal from the new $\mathrm{D}_{2} \mathrm{O}$-substituted complex; the labeled $\mathrm{Z}$ represents the signal from the pyridyl $\mathrm{C}-\mathrm{H}$ of the pyridine ring that is trans to the imidazole released (B) Schematic representation of ligand exchange of complex $\mathbf{3}$

A.

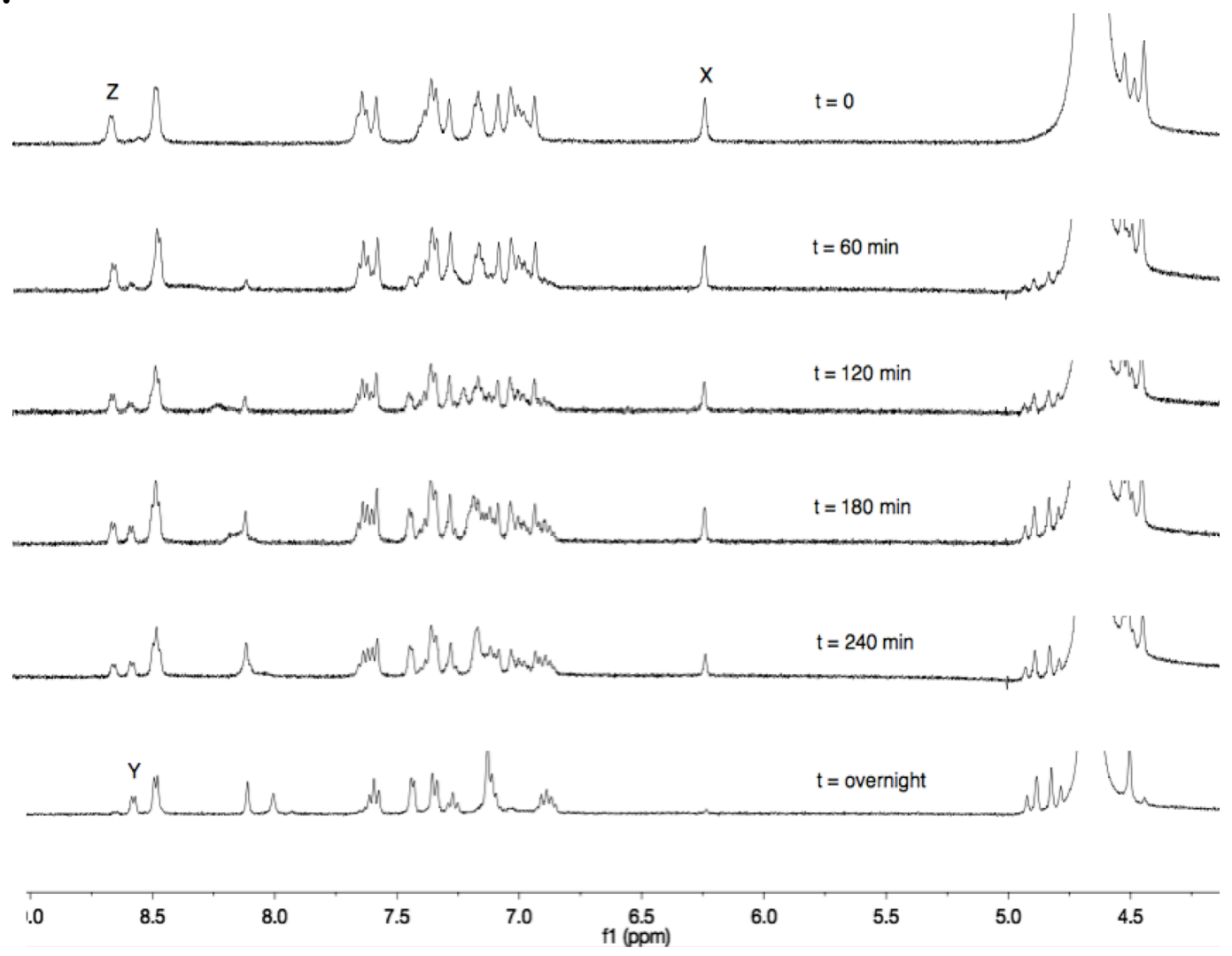

B.

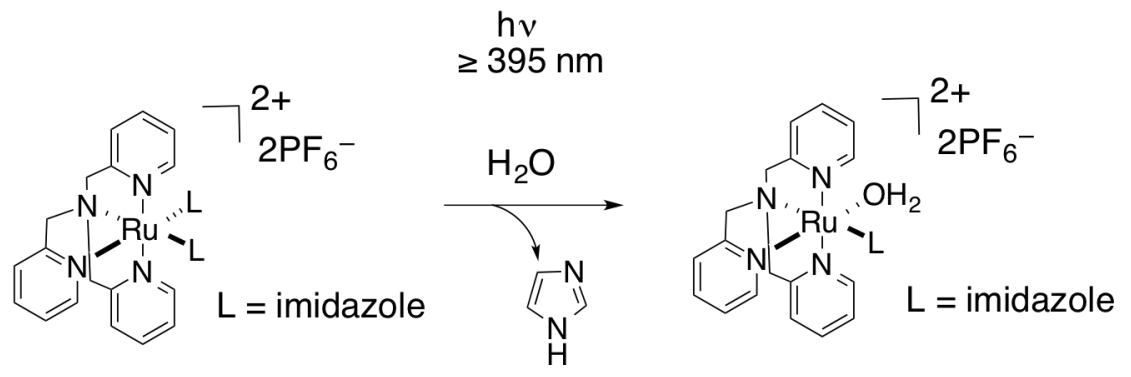


A.

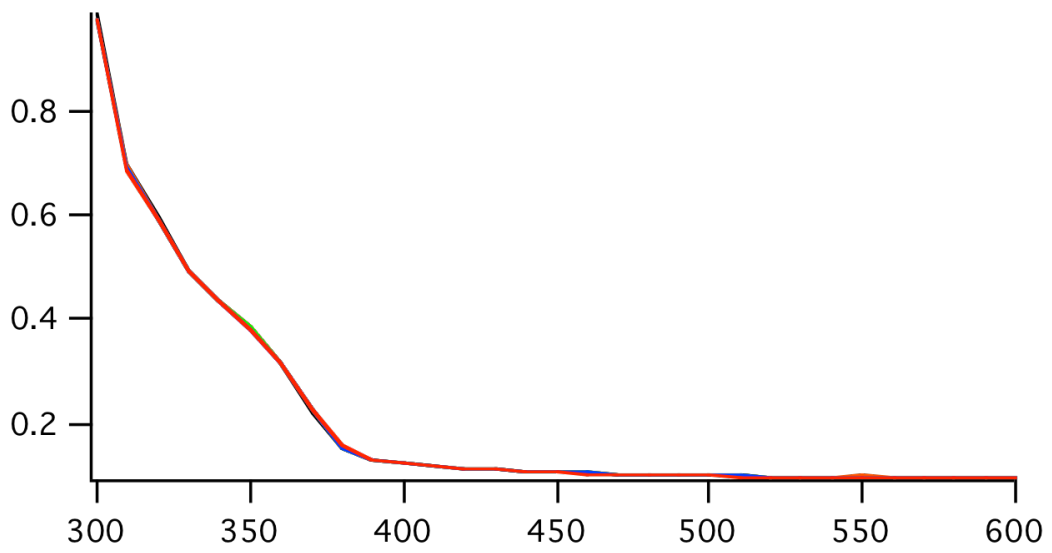

B.

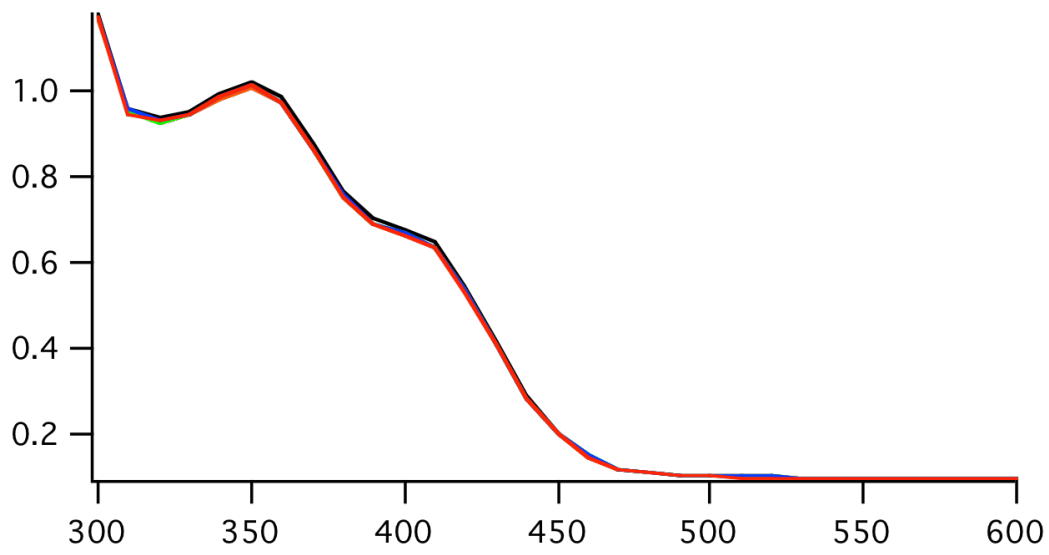

Figure S15. Absorbance scan for DMSO solvent control (A) and complex 1 (B) at $23.0^{\circ} \mathrm{C}$ in DMSO. The spectra were taken at $\mathrm{t}=0$ (black), 4 (orange), 8 (green), 12 (blue) and 24 (red) h. 
C.

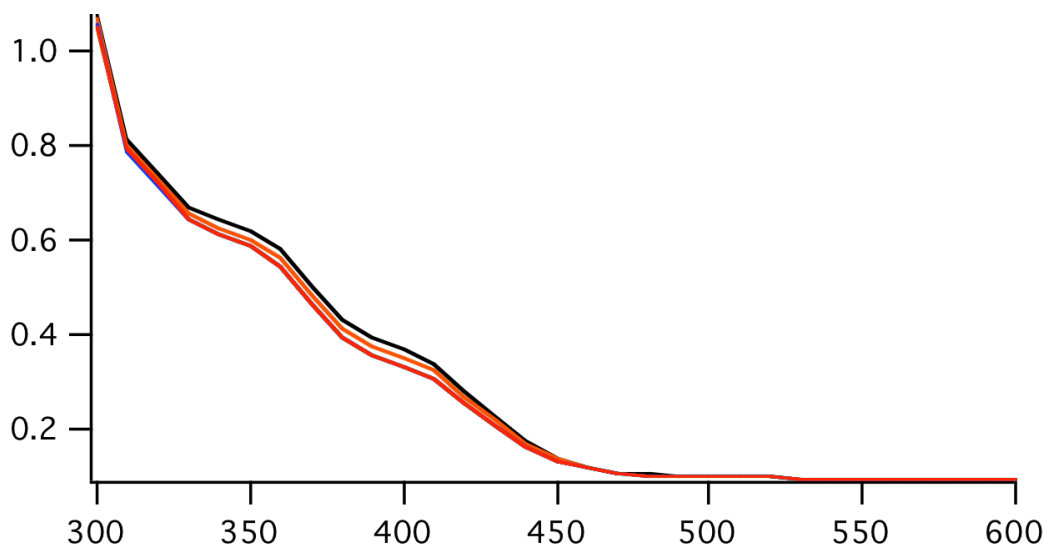

D.

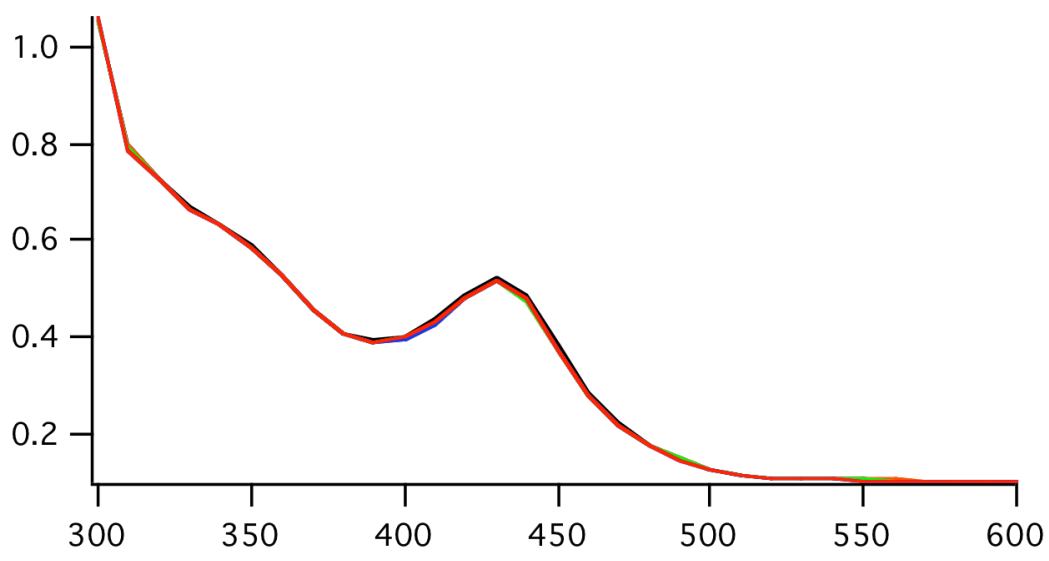

Figure S16. Absorbance scan for complex 2 (C) and 3 (D) at $23.0^{\circ} \mathrm{C}$ in DMSO. The spectra were taken at $\mathrm{t}=0$ (black), 4 (orange), 8 (green), 12 (blue) and 24 (red) h. 
E.

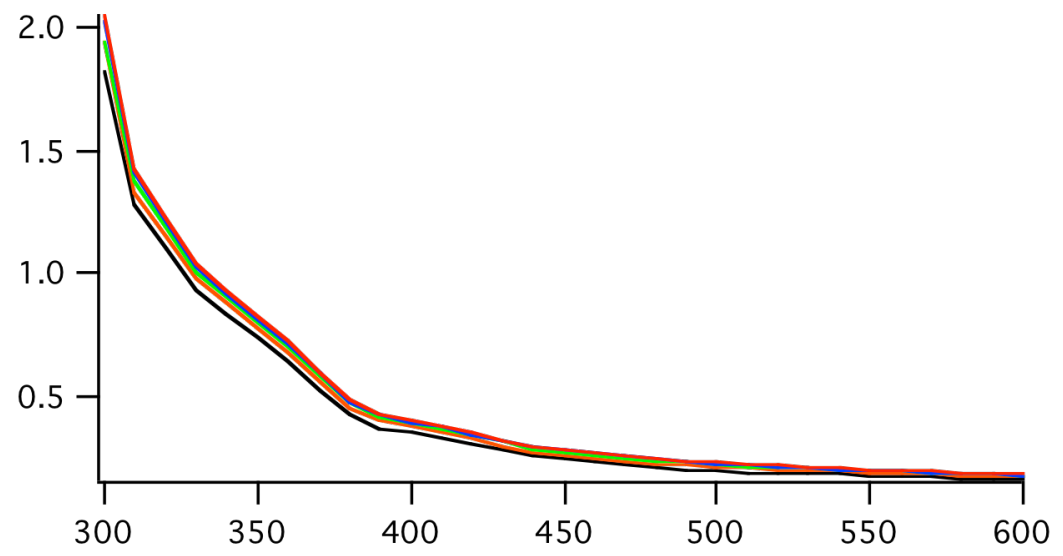

F.

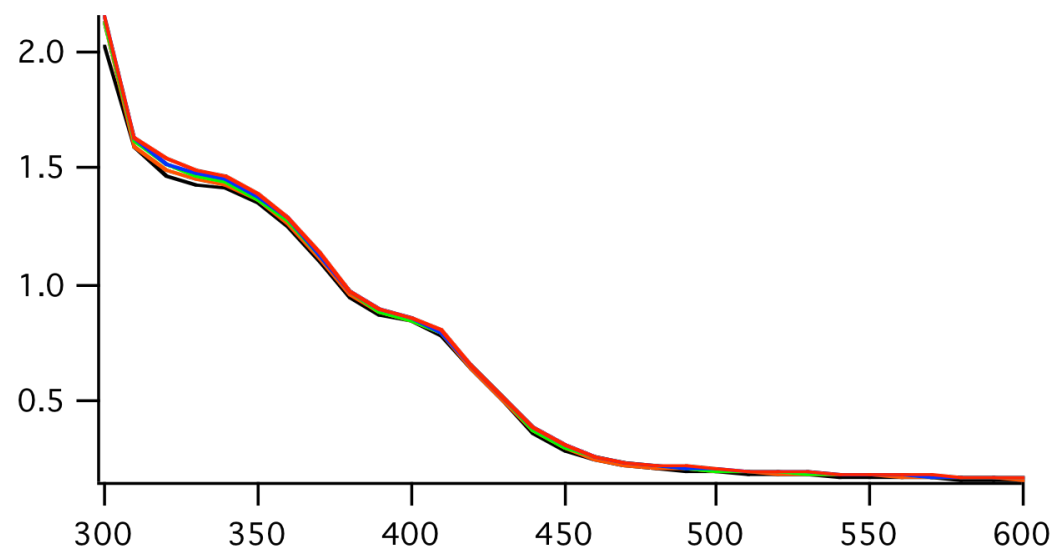

Figure S17. Absorbance scan for cell growth media control (E) and complex 1 (F) at $37.0^{\circ} \mathrm{C}$ in cell growth media $(<5 \%$ DMSO $)$. The spectra were taken at $\mathrm{t}=$ 0 (black), 4 (orange), 8 (green), 12 (blue) and 24 (red) h. 
G.

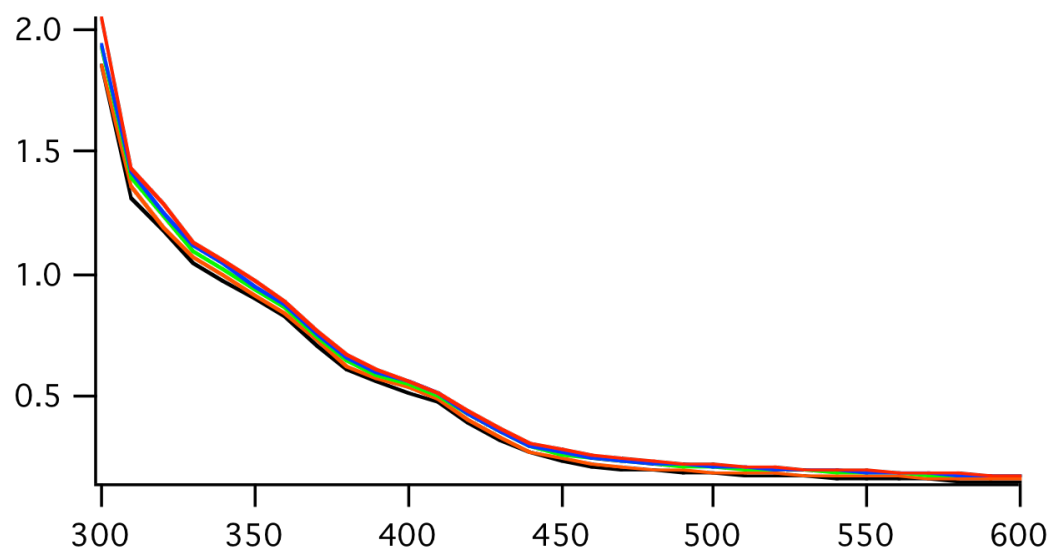

H.

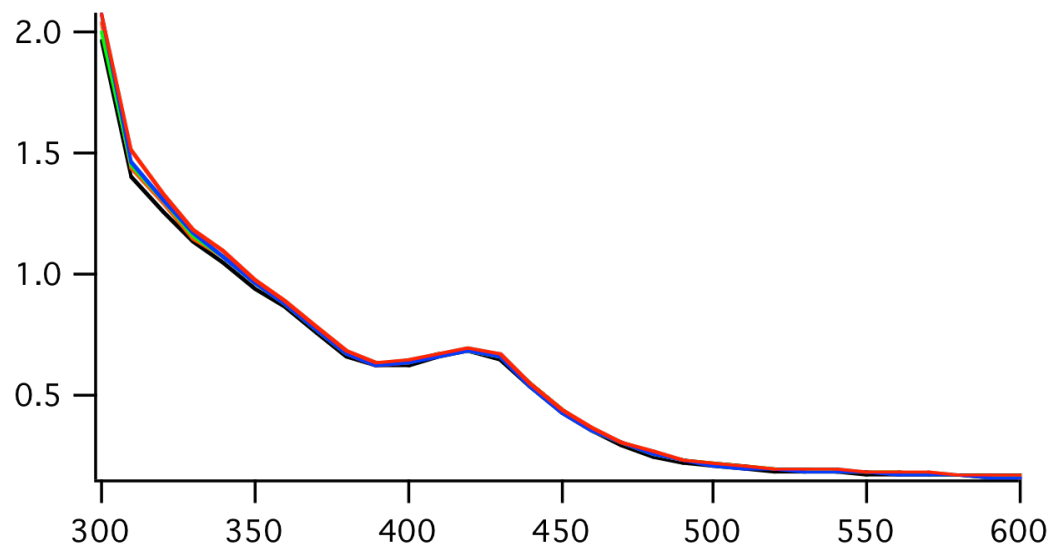

Figure S18. Absorbance scan for complex $2(\mathrm{G})$ and complex $3(\mathrm{H})$ at $37.0^{\circ} \mathrm{C}$ in cell growth media ( $<5 \%$ DMSO). The spectra were taken at $\mathrm{t}=0$ (black), 4 (orange), 8 (green), 12 (blue) and 24 (red) h. 
TD-DFT data calculated for complexes 1 and 3:

Figure S19. TD-DFT spectrum for the complex 1. The MLCT absorption bands found around $400 \mathrm{~nm}$ result from $\mathrm{HOMO} \rightarrow$ LUMO, HOMO- $\rightarrow$ LUMO, and HOMO-2 $\rightarrow$ LUMO (isodensity value of 0.05 a.u.) transitions. The HOMO, HOMO-1, and HOMO-2 are Rubased $\mathrm{d} \pi$ orbitals and LUMO is a pyridine-based $\pi *$ on the TPA ligand.

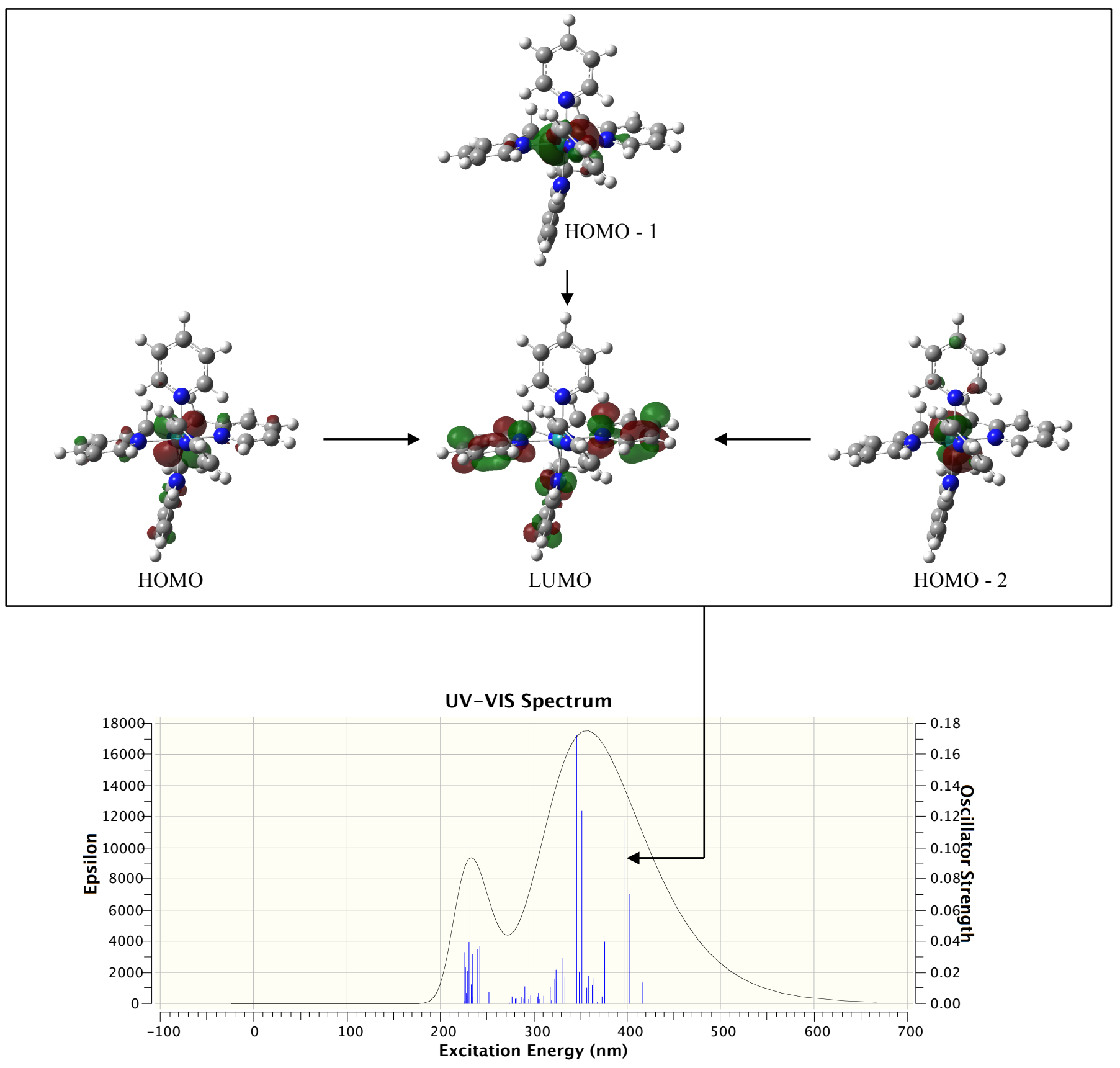


Figure S20. TD-DFT spectrum for the complex 3. The MLCT absorption band found around $400 \mathrm{~nm}$ results mainly from HOMO- $1 \rightarrow$ LUMO (isodensity value of 0.05 a.u.) transition. HOMO- 1 is Ru-based $\mathrm{d} \pi$ orbital and LUMO is a pyridine-based $\pi^{*}$ on the TPA ligand.

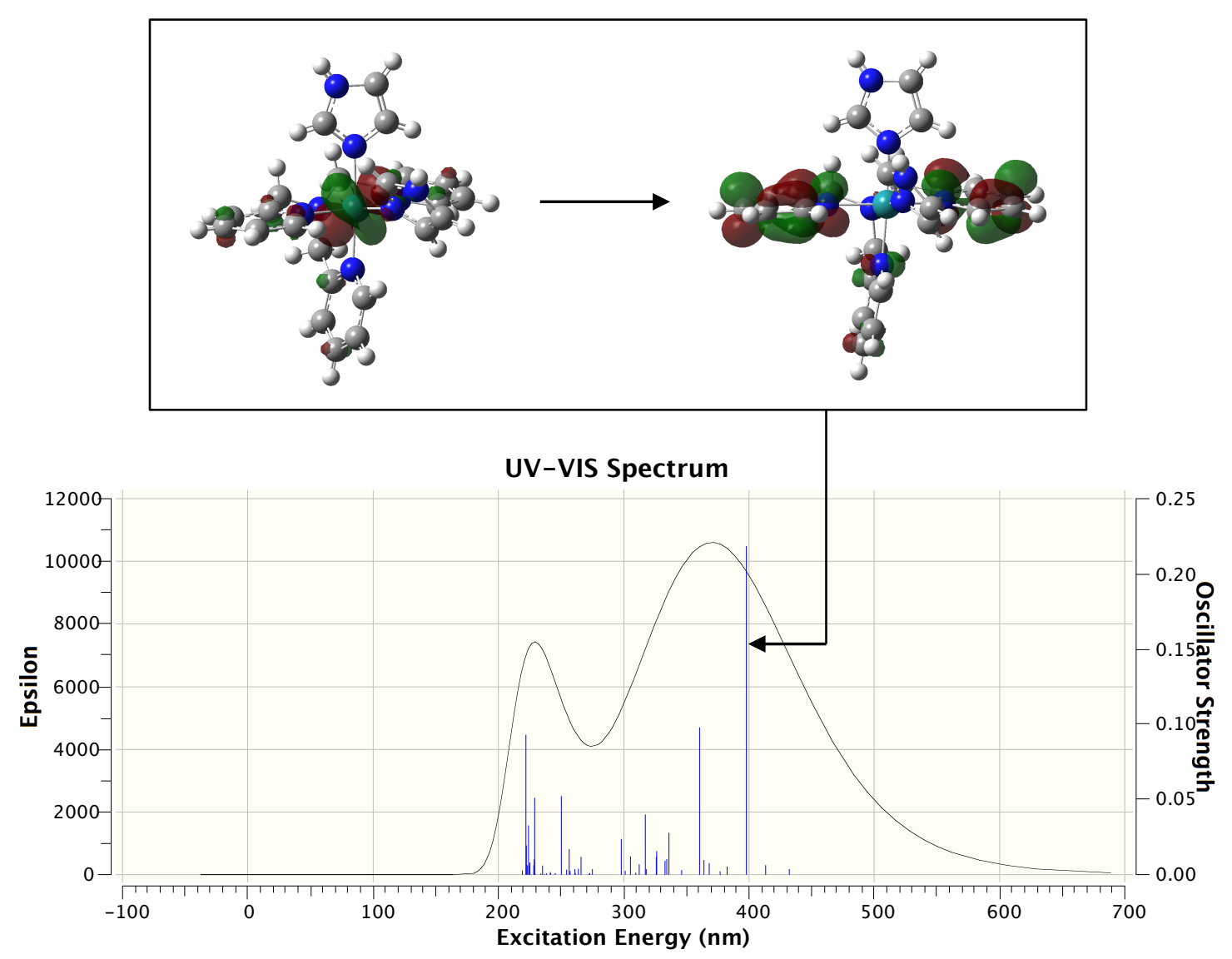




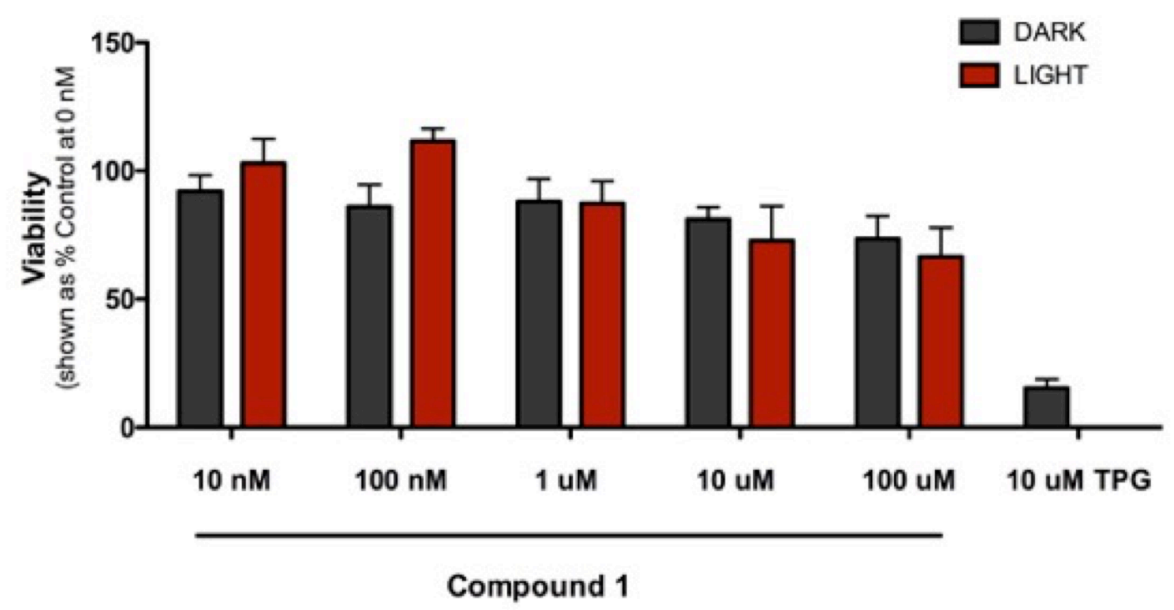

Figure S21. Cytoxicity of $\mathbf{1}$ in PC-3 prostate cancer cells. Cells were incubated in the presence of $1(10 \mathrm{nM}-100 \mathrm{mM})$ for $30 \mathrm{~min}$, left in the dark (black bars) or irradiated (red bars) for $40 \mathrm{~min}$ with a $250 \mathrm{~W}$ tungsten halogen lamp $\left(1_{\text {irr }}>395 \mathrm{~nm}\right)$. For comparison, cells were treated with the known cytotoxic agent thapsigargin (TPG). Cell viability was determined by MTT assay after $48 \mathrm{~h}$, and is reported relative to control with only vehicle (0.1\% DMSO) added. Error bars represent the standard error of the mean of quadruplicate wells, and data are representative of three independent experiments. 


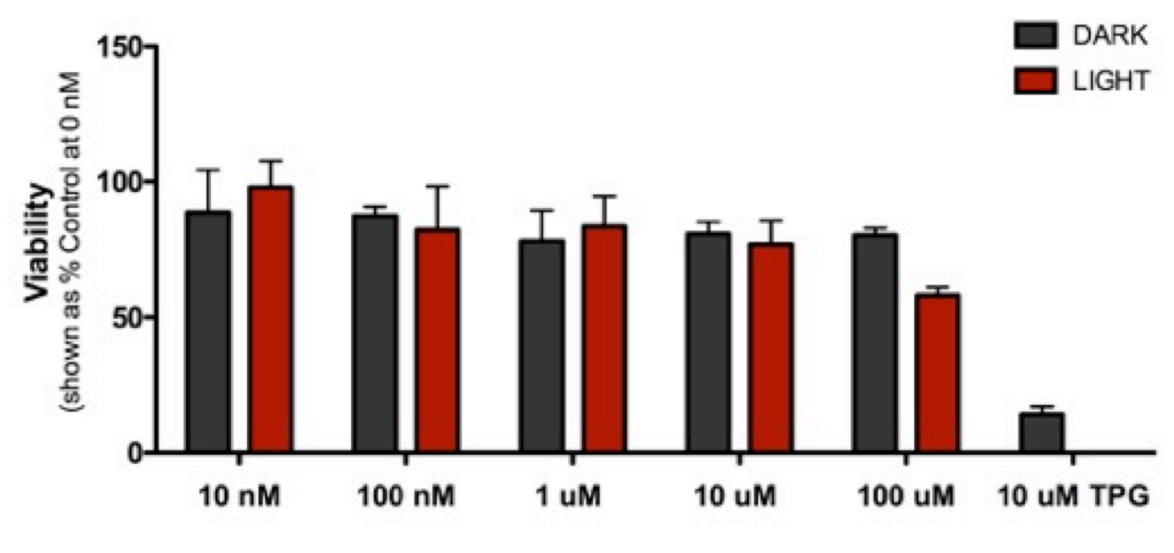

Compound 3

Figure S22. Cytoxicity of $\mathbf{3}$ in PC-3 prostate cancer cells. Cells were incubated in the presence of $\mathbf{3}(10 \mathrm{nM}-100 \mathrm{mM})$ for $30 \mathrm{~min}$, left in the dark (black bars) or irradiated (red bars) for 40 min with a $250 \mathrm{~W}$ tungsten halogen lamp $\left(1_{\text {irr }}>395 \mathrm{~nm}\right)$. For comparison, cells were treated with the known cytotoxic agent thapsigargin (TPG). Cell viability was determined by MTT assay after $48 \mathrm{~h}$, and is reported relative to control with only vehicle $(0.1 \%$ DMSO) added. Error bars represent the standard error of the mean of quadruplicate wells, and data are representative of three independent experiments. 


\section{References:}

(1) Becke, A. D. J. Chem. Phys. 1993, 98, 5648-5652.

(2) Perdew, J. P. Phys. Rev. B 1986, 33, 8822.

(3) Perdew, J. P. B., K.; Wang, Y. Phys. Rev. B 1996, 54, 16533.

(4) Frisch, M. J.; Trucks, G. W.; Schlegel, H. B.; Scuseria, G. E.; Robb, M. A.; Cheeseman, J. R.; Scalmani, G.; Barone, V.; Mennucci, B.; Petersson, G. A.; Nakatsuji, H.; Caricato, M.; Li, X.; Hratchian, H. P.; Izmaylov, A. F.; Bloino, J.; Zheng, G.; Sonnenberg, J. L.; Hada, M.; Ehara, M.; Toyota, K.; Fukuda, R.; Hasegawa, J.; Ishida, M.; Nakajima, T.; Honda, Y.; Kitao, O.; Nakai, H.; Vreven, T.; Montgomery, J. A., Jr.; Peralta, J. E.; Ogliaro, F.; Bearpark, M.; Heyd, J. J.; Brothers, E.; Kudin, K. N.; Staroverov, V.; Kobayashi, R.; Normand, J.; Raghavachari, K.; Rendell, A.; Burant, J. C.; Iyengar, S. S.; Tomasi, J.; Cossi, M.; Rega, N.; Millam, J. M.; Klene, M.; Knox, J. E.; Cross, J. B.; Bakken, V.; Adamo, C.; Jaramillo, J.; Gomperts, R.; Stratmann, R. E.; Yazyev, O.; Austin, A. J.; Cammi, R.; Pomelli, C.; Ochterski, J. W.; Martin, R. L.; Morokuma, K.; Zakrzewski, V. G.; Voth, G. A.; Salvador, P.; Dannenberg, J. J.; Dapprich, S.; Parandekar, P. V.; Mayhall, N. J.; Daniels, A. D.; Farkas, O.; Foresman, J. B.; Ortiz, J. V.; Cioslowski, J.; Fox, D. J.; Revision H.01 ed.; Gaussian, Inc.: Wallingford, CT, 2009.

(5) Andrae, D.; Häußermann, U.; Dolg, M.; Stoll, H.; Preuß, H. Theoret. Chim. Acta 1990, 77, 123-141.

(6) Igel-Mann, G.; Stoll, H.; Preuss, H. Mol. Phys. 1988, 65, 1321-1328.

(7) Dunning, T. H., Jr.; Hay, P. J. In Methods of Electronic Structure Theory; Schaefer, H., III, Ed.; Springer US: 1977; Vol. 3, p 1-27.

(8) Francl, M. M. J. Chem. Phys 1982, 77, 3654.

(9) Hariharan, P. C.; Pople, J. A. Theoret. Chim. Acta 1973, 28, 213-222.

(10) Marenich, A. V. C., C. J.; Truhlar, D. G. J. Phys. Chem. B 2009, 113, 6378-6396.

(11) Petit, L. M., P.; Adamo, C. J. Chem. Theory Comput. 2005, 1, 953-962.

(12) Runge, E.; Gross, E. K. U. Phys. Rev. Lett.1984, 52, 997-1000.

(13) Stratmann, R. E.; Scuseria, G. E.; Frisch, M. J. J. Chem. Phys 1998, 109, 8218-8224.

(14) Sharma, R.; Knoll, J. D.; Martin, P. D.; Podgorski, I.; Turro, C.; Kodanko, J. J. Inorg . Chem. 2014, 53, 3272-3274.

(15) Montalti, M.; Credi, A.; Prodi, L.; Gandolfi, M. T. Handbook of Photochemistry; 3rd ed.; CRC Press: Boca Raton, FL, 2006.

(16) Kaighn, M. E.; Narayan, K. S.; Ohnuki, Y.; Lechner, J. F.; Jones, L. W. Invest. Urol. 1979, 17, 16-23.

(17) Respondek, T.; Sharma, R.; Herroon, M. K.; Garner, R. N.; Knoll, J. D.; Cueny, E.; Turro, C.; Podgorski, I.; Kodanko, J. J. ChemMedChem 2014, 9, 1306-1315. 